\title{
Molecular Iodine-Mediated Chemoselective Synthesis of Multi-substituted Pyridines through Catabolism and Reconstruction Behavior of Natural Amino Acids
}

\author{
Jiachen Xiang, Miao Wang, Yan Cheng, Anxin Wu* \\ Key Laboratory of Pesticide \& Chemical Biology, Ministry of Education, Central China Normal \\ University, Wuhan 430079, P. R. China \\ E-mail: chwuax@mail.ccnu.edu.cn
}

Table of Contents page

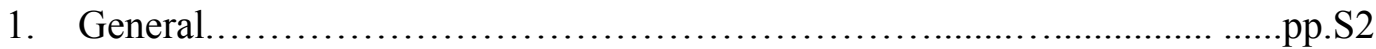

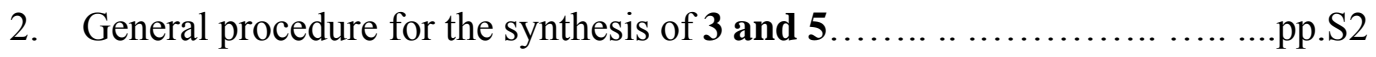

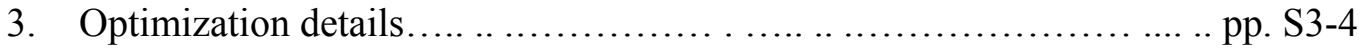

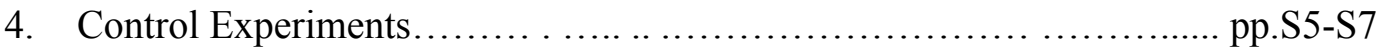

5. Spectra for labeling and control experiments.................... pp.S8-S11

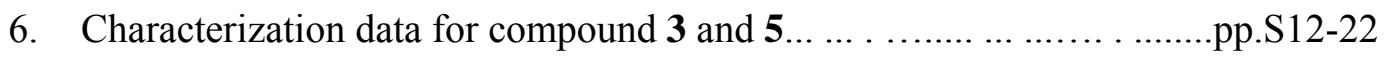

7. Crystallographic data and molecular structure of compound $3 \mathbf{h}$.............pp.S23

8. Copies of ${ }_{1} \mathbf{H}$ NMR , ${ }_{13}$ C NMR and HRMS/MS Spectra.. ... ............pp.S24-52 


\section{General}

All substrates and reagents were commercially available and used without further purification. TLC analysis was performed using pre-coated glass plates. Column chromatography was performed using alkaline aluminum oxide (200-300 mesh). IR spectra were recorded on a Perkin-Elmer PE-983 infrared spectrometer as $\mathrm{KBr}$ pellets with absorption in $\mathrm{cm}-1.1 \mathrm{H}$ spectra were recorded in $\mathrm{CDCl}_{3}$ or DMSO- $d_{6}$ or $\mathrm{CD}_{3} \mathrm{COCD}_{3}$ on $400 / 600 \mathrm{MHz}$ NMR spectrometers and resonances $(\delta)$ are given in parts per million relative to tetramethylsilane. Data are reported as follows: chemical shift, multiplicity $(\mathrm{s}=$ singlet, $\mathrm{d}=$ doublet, $\mathrm{t}=$ triplet, $\mathrm{m}=$ multiplet $\mathrm{q}=$ quadruple), coupling constants $(\mathrm{Hz})$ and integration. ${ }_{13} \mathrm{C}$ spectra were recorded in $\mathrm{CDCl}_{3}$ or DMSO- $d 6$ on $100 / 150 \mathrm{MHz}$ NMR spectrometers and resonances $(\delta)$ are given in ppm. HRMS were obtained on a Bruker 7-tesla FT-ICR MS equipped with an electrospray source. The X-ray crystal structure determinations of $\mathbf{3 h}$ were obtained on a Bruker SMART APEX CCD system. Melting points were determined using XT-4 apparatus and not corrected.

\section{General procedure for the synthesis of 3 and 5 (3a and $5 a$ as an example)}

3a: A mixture of glycine 1a $(2.0 \mathrm{mmol})$, acetophenone $2 \mathbf{a}(2.0 \mathrm{mmol}), \mathrm{I}_{2}(2.0 \mathrm{mmol})$, in pyridine $(2.0 \mathrm{~mL})$ was stirred at $120{ }^{\circ} \mathrm{C}$ for 12 hours in a pressure vessel. Then added $50 \mathrm{~mL}$ water and 30 $\mathrm{mL}$ saturated brine solution to the mixture and extracted with EtOAc 3 times $(3 \times 50 \mathrm{~mL})$. The extract was washed with $10 \% \mathrm{Na}_{2} \mathrm{~S}_{2} \mathrm{O}_{3}$ solution, dried over anhydrous $\mathrm{Na}_{2} \mathrm{SO}_{4}$ and concentrated under reduced pressure. The crude product was purified by column chromatography on alkaline aluminum oxide (eluent: $\mathrm{n}$-hexane $/ \mathrm{EtOAc}=50 / 1$ ) to afford the product $3 \mathbf{a}$ as White crystalline solid (168 mg, 73\%).

5a: A mixture of $4 \mathrm{a}(2.0 \mathrm{mmol}), \mathrm{HOAc}(2.0 \mathrm{mmol}), \mathrm{I}_{2}(1.0 \mathrm{mmol}), \mathrm{DMSO}(3.0 \mathrm{ml})$ was stirred at $115{ }^{\circ} \mathrm{C}$ for 12 hours in a pressure vessel. Then added $50 \mathrm{~mL}$ water and $30 \mathrm{~mL}$ saturated brine solution to the mixture, extracted with EtOAc 3 times $(3 \times 50 \mathrm{~mL})$. The extract was washed with $10 \% \mathrm{Na}_{2} \mathrm{~S}_{2} \mathrm{O}_{3}$ solution, dried over anhydrous $\mathrm{Na}_{2} \mathrm{SO}_{4}$ and concentrated under reduced pressure. The crude product was purified by column chromatography on alkaline aluminum oxide (eluent: petroleum ether $/ \mathrm{EtOAc}=2 / 1)$ to afford the product $5 \mathbf{a}$ as black crystalline solid $(116 \mathrm{mg}, 75 \%)$. 


\section{Optimization details of reaction $\mathbf{1}^{a}$}

\begin{tabular}{|c|c|c|c|c|c|}
\hline entry & solvent & $\begin{array}{c}\mathrm{I}_{2} \\
\text { (eq.) }\end{array}$ & Additive & $\begin{array}{l}\text { temp } \\
\left({ }^{\circ} \mathbf{C}\right)\end{array}$ & $\begin{array}{c}\text { Yield }^{b} \\
(\%)\end{array}$ \\
\hline 1 & DMF & 1.0 & DBU (2.0 eq.) & 100 & trace \\
\hline 2 & DMF & 1.0 & DMAP (2.0 eq.) & 100 & trace \\
\hline 3 & DMF & 1.0 & $\mathrm{DABCO}$ (2.0 eq.) & 100 & 0 \\
\hline 4 & DMF & 1.0 & Pyridine ( 2.0 eq.) & 100 & $31 \%$ \\
\hline 5 & DMF & 1.0 & $\mathrm{Cs}_{2} \mathrm{CO}_{3}(1.0$ eq. $)$ & 100 & trace \\
\hline 6 & DMF & 1.0 & $\mathrm{~K}_{2} \mathrm{CO}_{3}$ (1.0 eq. $)$ & 100 & 0 \\
\hline 7 & DMF & 1.0 & $\mathrm{KOH}$ (2.0 eq.) & 100 & 0 \\
\hline 8 & DMF & 1.0 & $t$-BuOK (2.0 eq.) & 100 & 0 \\
\hline 9 & DMF & 1.0 & $\mathrm{~K}_{3} \mathrm{PO}_{4}(1.0$ eq. $)$ & 100 & 0 \\
\hline 10 & DMF & 1.0 & - & 100 & 0 \\
\hline 11 & Pyridine & 1.0 & - & 100 & $61 \%$ \\
\hline 12 & Pyridine & 1.0 & DBU (2.0 eq.) & 100 & trace \\
\hline 13 & Pyridine & 1.0 & DMAP (2.0 eq.) & 100 & $36 \%$ \\
\hline 14 & Pyridine & 1.0 & $\mathrm{Cs}_{2} \mathrm{CO}_{3}(1.0$ eq. $)$ & 100 & trace \\
\hline 15 & Pyridine & 1.0 & $\mathrm{~K}_{2} \mathrm{CO}_{3}$ (1.0 eq.) & 100 & $23 \%$ \\
\hline 16 & Pyridine & 1.0 & $\mathrm{KOH}$ (2.0 eq.) & 100 & $27 \%$ \\
\hline 17 & Pyridine & 1.0 & - & 110 & $65 \%$ \\
\hline 18 & Pyridine & 1.0 & - & 120 & $73 \%$ \\
\hline 19 & Pyridine & 0.2 & - & 120 & $24 \%$ \\
\hline 20 & Pyridine & 0.5 & - & 120 & $41 \%$ \\
\hline 21 & Pyridine & 1.5 & - & 120 & $27 \%$ \\
\hline
\end{tabular}

${ }^{a}$ Reaction conditions: 1a $(2.0 \mathrm{mmol}$, excessive dose $)$, 2a $(2.0 \mathrm{mmol})$, Addictive, solvent $(2.0 \mathrm{~mL})$ for $12 \mathrm{~h} .{ }^{b}$ isolated yields based on 2. Reactions were carried out in a pressure vessel. 
3. Optimization details of reaction $2^{a}$

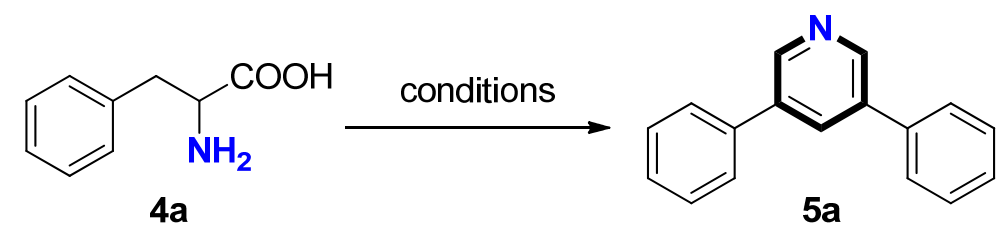

\begin{tabular}{|c|c|c|c|c|c|c|}
\hline entry & solvent & $\begin{array}{c}\mathrm{I}_{2} \\
\text { (equiv) }\end{array}$ & $\begin{array}{c}\text { additive } \\
\text { (1.0 equiv) }\end{array}$ & $\begin{array}{l}\text { temp. } \\
\left({ }^{\circ} \mathrm{C}\right)\end{array}$ & $\begin{array}{r}\text { time } \\
\text { (h) }\end{array}$ & $\begin{array}{r}\text { yield }^{b} \\
(\%)\end{array}$ \\
\hline 1 & $\mathrm{CH}_{3} \mathrm{CN}$ & 1.0 & - & reflux & 12 & trace \\
\hline 2 & DMSO & 1.0 & - & 80 & 12 & 15 \\
\hline 3 & DMF & 1.0 & - & 80 & 12 & 11 \\
\hline 4 & THF & 1.0 & - & 80 & 12 & 0 \\
\hline 5 & dioxane & 1.0 & - & 80 & 12 & trace \\
\hline 6 & $i-\mathrm{PrOH}$ & 1.0 & - & reflux & 12 & 0 \\
\hline 7 & $\mathrm{H}_{2} \mathrm{O}$ & 1.0 & - & 80 & 12 & 0 \\
\hline 8 & DMSO & 1.0 & - & 100 & 6 & 31 \\
\hline 9 & DMSO & 1.0 & - & 115 & 6 & 46 \\
\hline 10 & DMSO & 0.2 & - & 115 & 6 & 20 \\
\hline 11 & DMSO & 0.4 & - & 115 & 6 & 25 \\
\hline 12 & DMSO & 0.2 & - & 115 & 24 & 65 \\
\hline 13 & DMSO & 0.4 & - & 115 & 24 & 68 \\
\hline 14 & DMSO & 0.5 & - & 115 & 6 & 61 \\
\hline 15 & DMSO & 1.0 & - & 115 & 6 & 46 \\
\hline 16 & DMSO & 1.2 & - & 115 & 6 & 29 \\
\hline 17 & DMSO & 1.5 & - & 115 & 6 & $<10$ \\
\hline 18 & DMSO & 0.5 & KOAc & 115 & 6 & trace \\
\hline 19 & DMSO & 0.5 & $\mathrm{~K}_{2} \mathrm{CO}_{3}$ & 115 & 6 & 25 \\
\hline 20 & DMSO & 0.5 & $\mathrm{~K}_{3} \mathrm{PO}_{4}$ & 115 & 6 & $<10$ \\
\hline 21 & DMSO & 0.5 & $\mathrm{NaOAc}$ & 115 & 6 & trace \\
\hline 22 & DMSO & 0.5 & HOAC & 115 & 6 & 75 \\
\hline 23 & DMSO & 0.5 & acetic anhydride & 115 & 6 & 70 \\
\hline 24 & DMSO & 0.5 & TFA & 115 & 6 & trace \\
\hline 25 & DMSO & 0.5 & triflic acid & 115 & 6 & trace \\
\hline
\end{tabular}

${ }^{a}$ Reaction conditions: 4 a $(1.0 \mathrm{mmol}), \mathrm{I}_{2}$, addictive, solvent $(3.0 \mathrm{~mL}) .{ }^{b}$ isolated yields. Reactions were carried out in a pressure vessel. 


\section{Control Experiments.}

\subsection{For reaction 1.}

1 acetophenone was treated with 1.0 equivalent of $\mathrm{I}_{2}$ in the absence of an amino acid in pyridine under the standard condition for $6 \mathrm{~h}$. This reaction produced 1-(2-oxo-2-phenylethyl)pyridin-1-ium iodide (A) as a undissolved product in excellent yield. (Figure S1 a)

2 Given that the generation of the pyridinium ylide equivalent $\mathbf{A}$ would be an inevitable part of this reaction and pyridine was found to be critical to the success of this transformation, it seemed reasonable to assume that a pyridinium ylide was formed as a crucial intermediate during this reaction. The formation of $\mathbf{3 q}$ in $71 \%$ yield from the reaction of amino acid $\mathbf{1 q}$ with $\mathbf{A}$ in the absence of acetophenone provided further proof of this idea. However, that the yield of this reaction was lower than that of the reaction shown in Scheme 3, which indicated that acetophenone played another role in the transformation. (Figure S1 b)

3 The subsequent reaction of $1 \mathrm{q}$ with equal amounts of $\mathrm{A}$ and $\mathbf{2 v}$ under the standard conditions gave the symmetrical products $\mathbf{3 q}$ and $\mathbf{3 v}$ in $\mathbf{7}$ and $34 \%$ yields, respectively. The cross-trapping product 3qv was also obtained, as predicted, in a 45\% yield. (Figure S1 c)

(a)

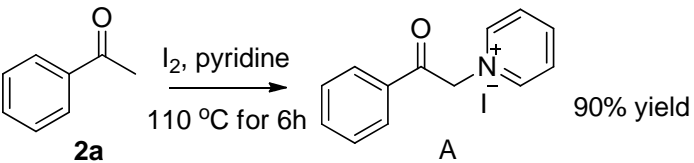

(b)
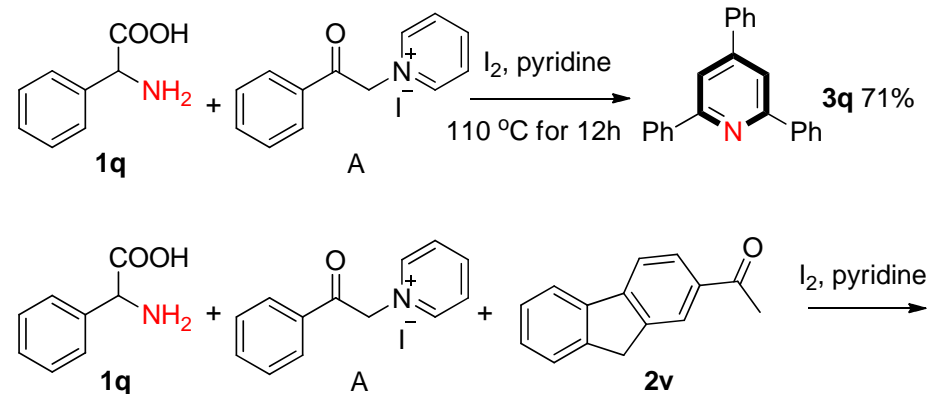

(c)

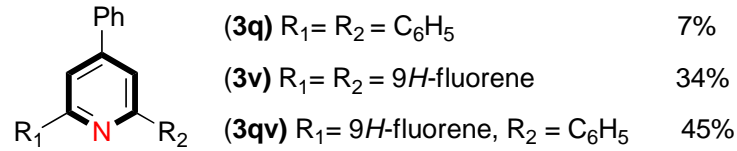

Figure $\mathrm{S} 1$

\subsection{For reaction 2.}

1. Add TEMPO (1.0 equiv) to the standard condition proved this transformation is not likely a free radical process. (Figure $\mathrm{S} 2$ a )

2. Then using phenylacetaldehyde and $\mathrm{NH}_{4} \mathrm{OAc}$ (3.0 equiv) to react under the current condition, 5 can be formed in 55\% yield, which determined that phenylacetaldehyde might be a key intermediate. (Figure S2 b )

3. Phenylethylamine could not work. (Figure S2 c)

4. Labeling experiments determined the $\mathrm{C}-\mathrm{N}$ cleavage process of phenylalanine. (Figure $\mathrm{S} 2 \mathrm{~d}$ )

5. We used GC-MS to monitor the reaction. possible byproduct 2-phenylacetonitrile (B3) could be detected which should be an over oxidation product of 2-phenylethanimine (B1), this can be one proof of the existence of unstable intermediate B1. (Figure S2 e) 
6. Toluene could also be found by GC-MS, which might be attributed to debenzylation/aromatization process. (Figure S2 e)

7. Possible condensation product $\mathbf{B} 4$ and aromatization byproduct $\mathbf{B} 5$ were detected by GC-MS as two important proofs in the transformation. (Figure S2 e)

8. In order to fit the requirement of our HRMS detection limitation (molecular weight larger than 121), we used 2-amino-3-(4-chlorophenyl)propanoic acid (4c) with larger molecular weight to do the test. $4 \mathrm{c}$ was reacted in standard condition for $1 \mathrm{~h}$, then we test the reaction mixture with HRMS, in addition to $\mathbf{5 c}$, we detected possible imine intermediate B1-5c, possible condensation intermediate B7-5c and byproduct B5-5c. Those compounds are all impartment supporting of our mechanism. (Figure S2 $\mathrm{f}$ )

9. The hydrolysis process of 2-phenylethanimine (B1) to phenylacetaldehyde (B2) is fast. We believe the subsequent formation of $\mathbf{5}$ make the hydrolysis process more easily to take place. That makes those unstable intermediate more difficult to be isolated. Once B2 generated in situ, it will react with other $\mathbf{B} 2$ or $\mathbf{B} 1$ quickly, so we cannot detect it. 
(a)

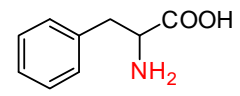

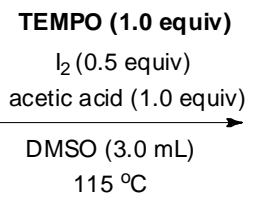

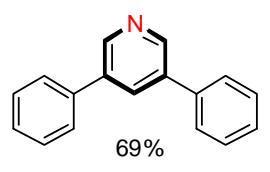

(b)

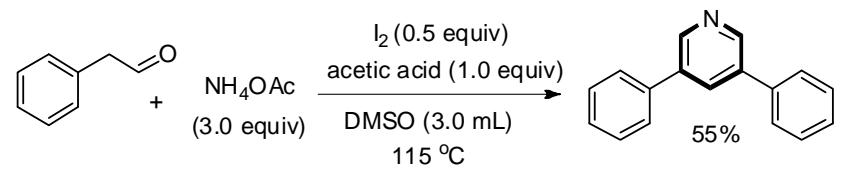

(c)

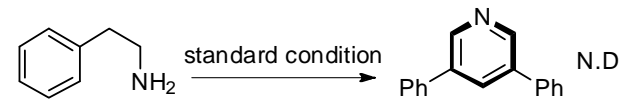

(d)

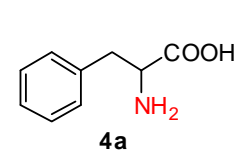

$4 a$

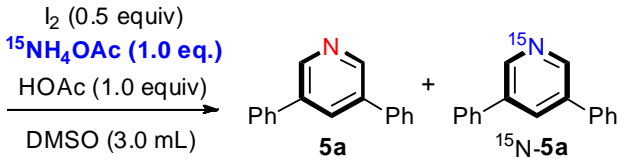
$115^{\circ} \mathrm{C}$

(e)<smiles>NC(Cc1ccccc1)C(=O)O</smiles>

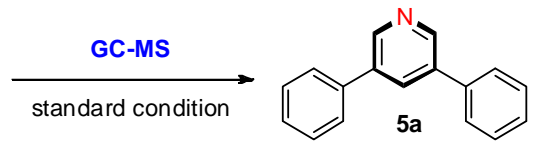

GC-MS detected

(possible structures):

$1>{ }_{N}$

1

B3

toluene
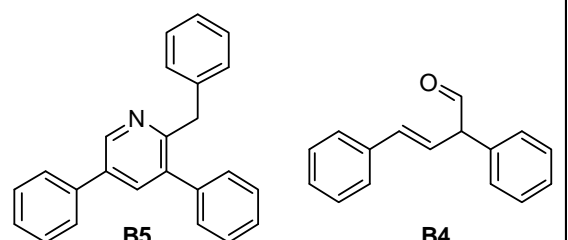

B4

(f)<smiles>NC(Cc1ccc(Cl)cc1)C(=O)O</smiles>
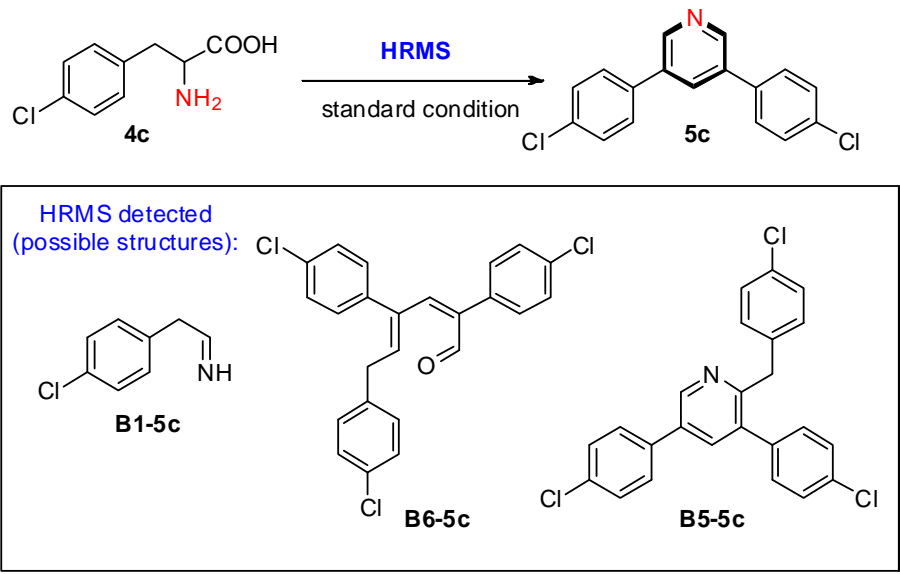

Figure S2

S7 
5. Spectra for labeling and control experiments.

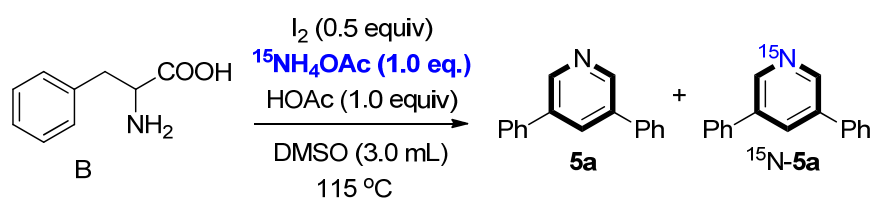

\section{1 labeling experiment detected by HRMS:}

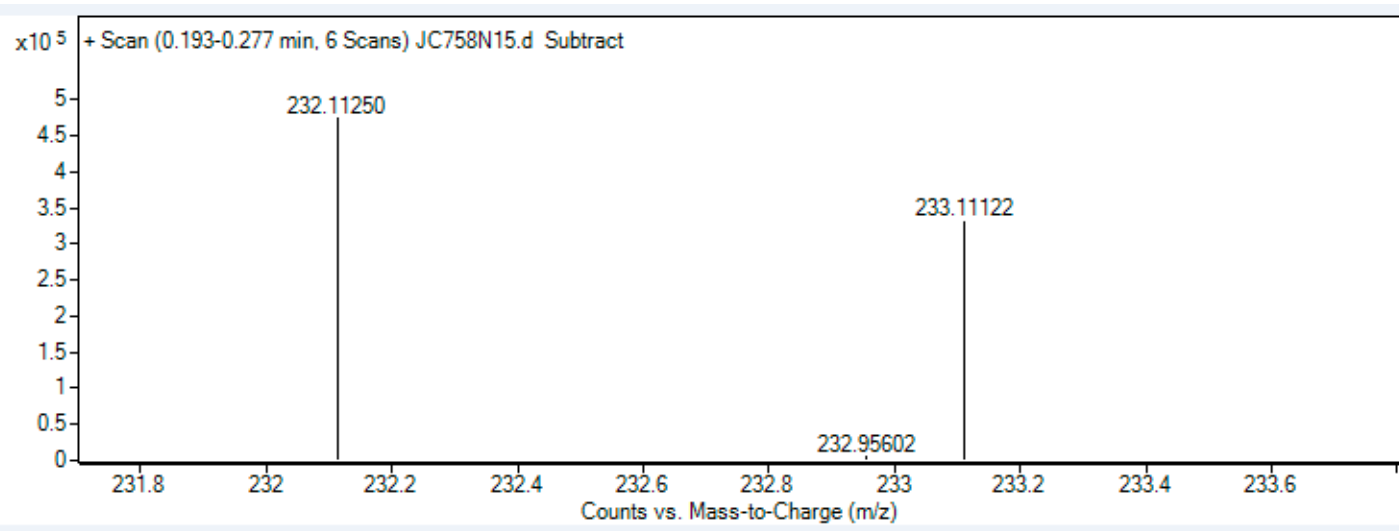

${ }^{14} \mathrm{~N}-5 a$, HRMS (ESI): $\mathrm{m} / \mathrm{z}[\mathrm{M}+\mathrm{H}]+$, found: 232.11250

${ }^{15} \mathrm{~N}-5 a$, HRMS (ESI): $\mathrm{m} / \mathrm{z}$ [M+H] ${ }^{+}$, found: 233.11122 
5.2 Reacted for $1 \mathrm{~h}$, then detected by HRMS, possible structures:
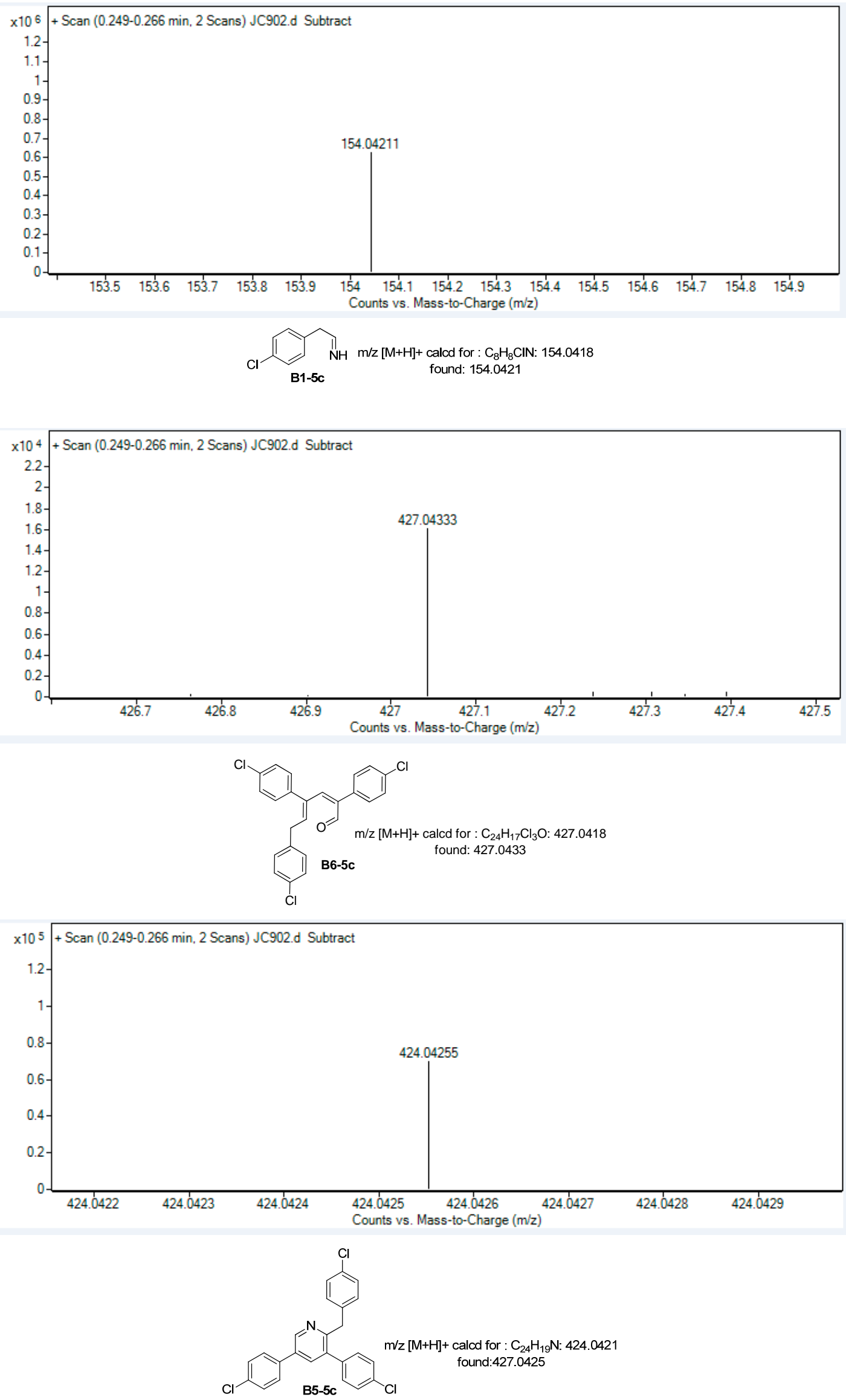


\subsection{Detected by GC-MS, possible structures:}

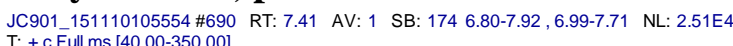
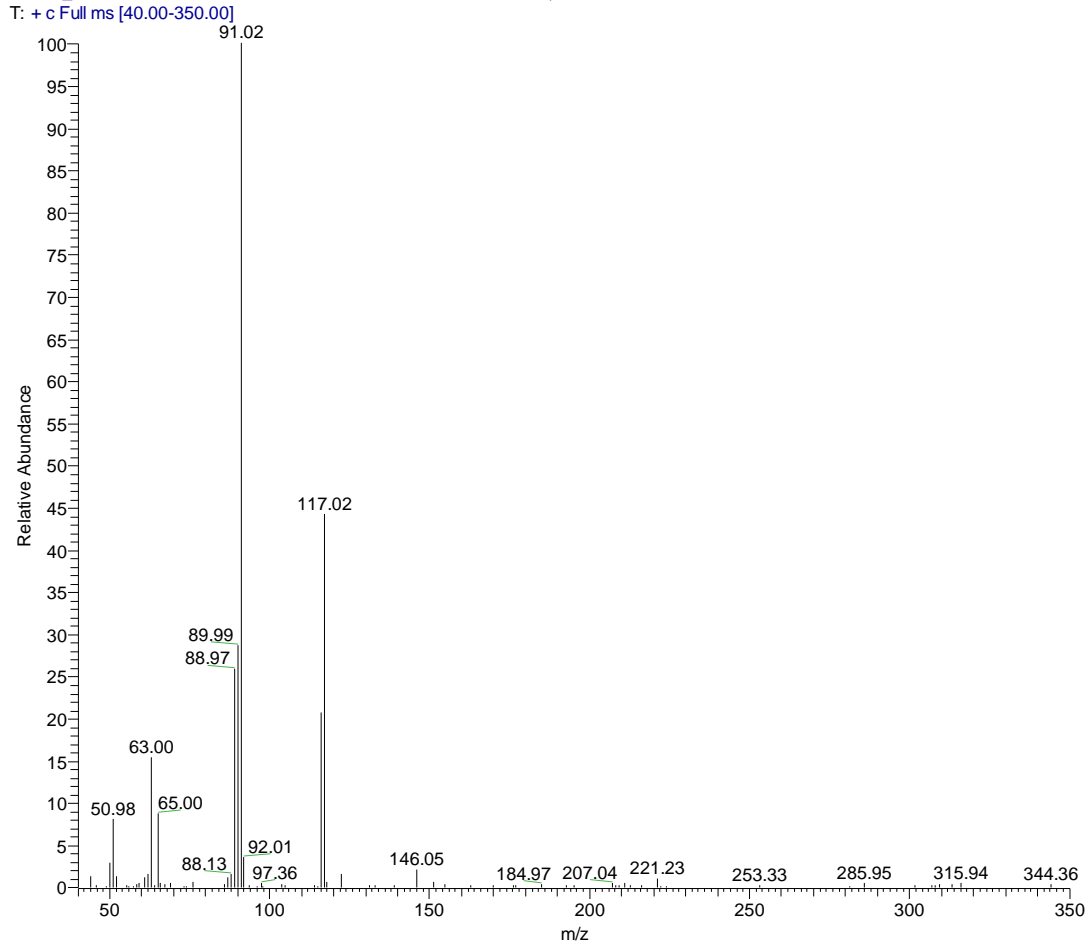

$\mathrm{B}_{\mathrm{N} 3} \begin{aligned} & \text { Chemical Formula: } \mathrm{C}_{8} \mathrm{H}_{7} \mathrm{~N} \\ & \text { Molecular Weight: } 117.15\end{aligned}$

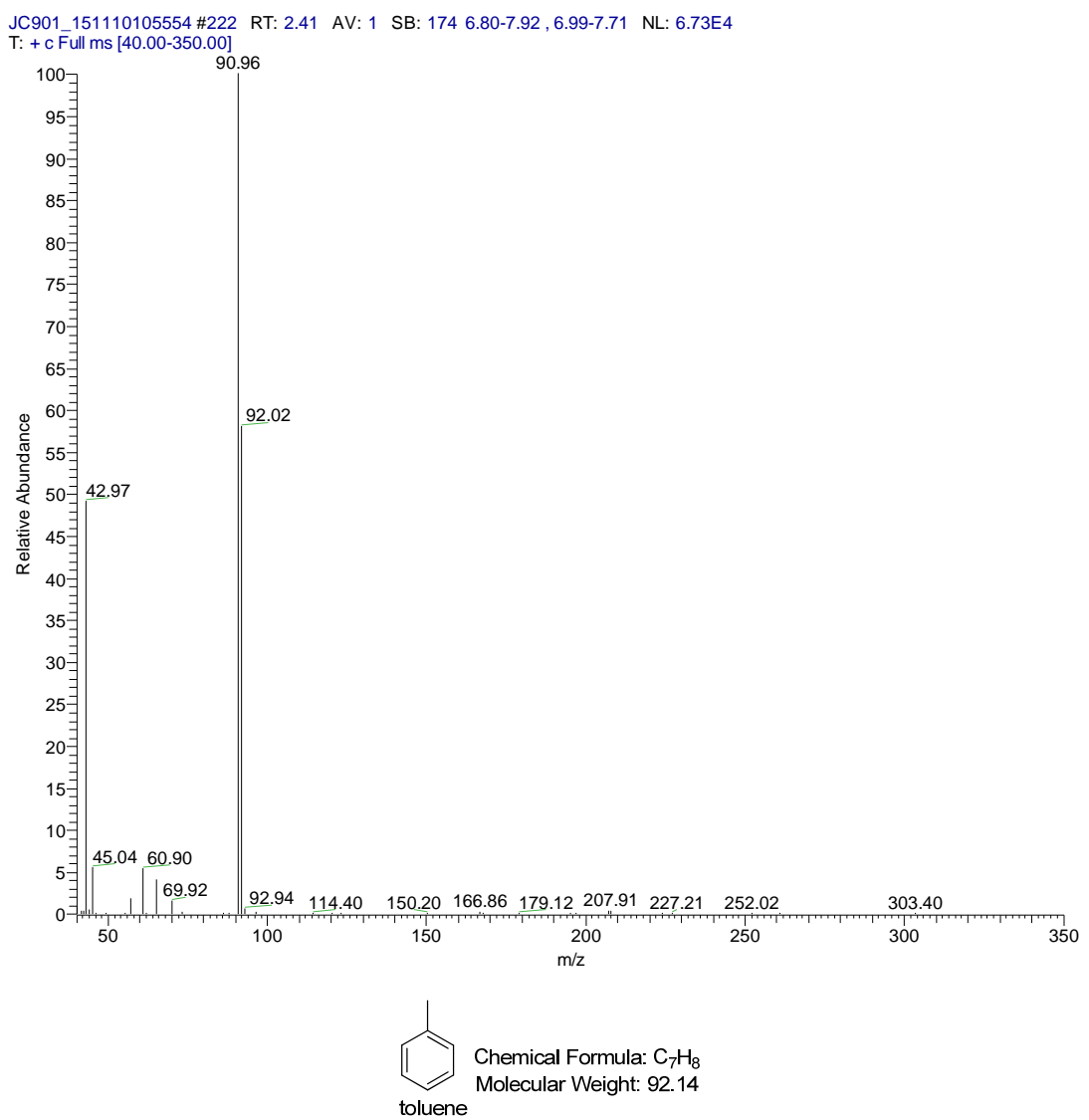




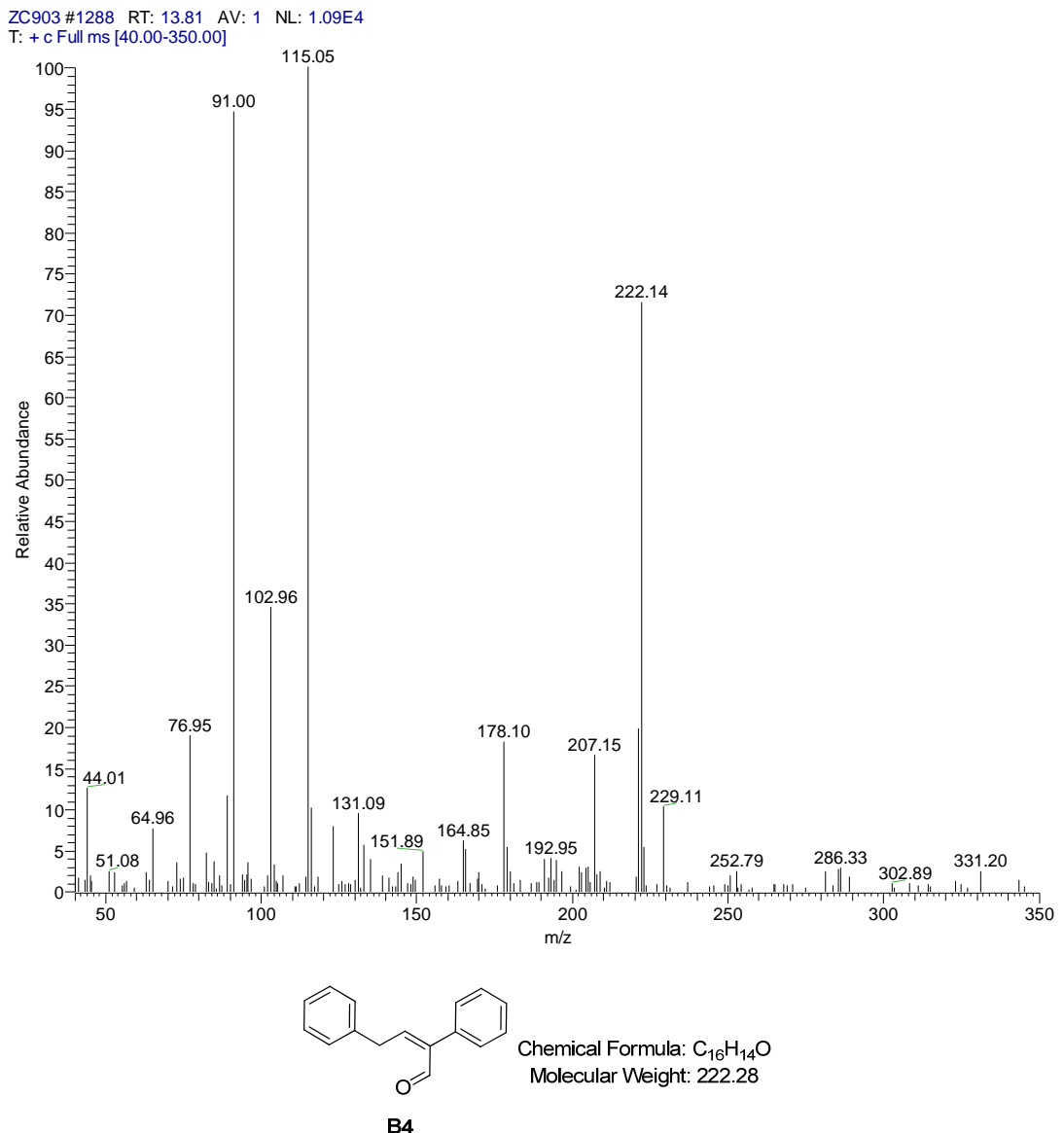

ZC904_151112102414\#936 RT: 13.00 AV: 1 NL: 3.82E4
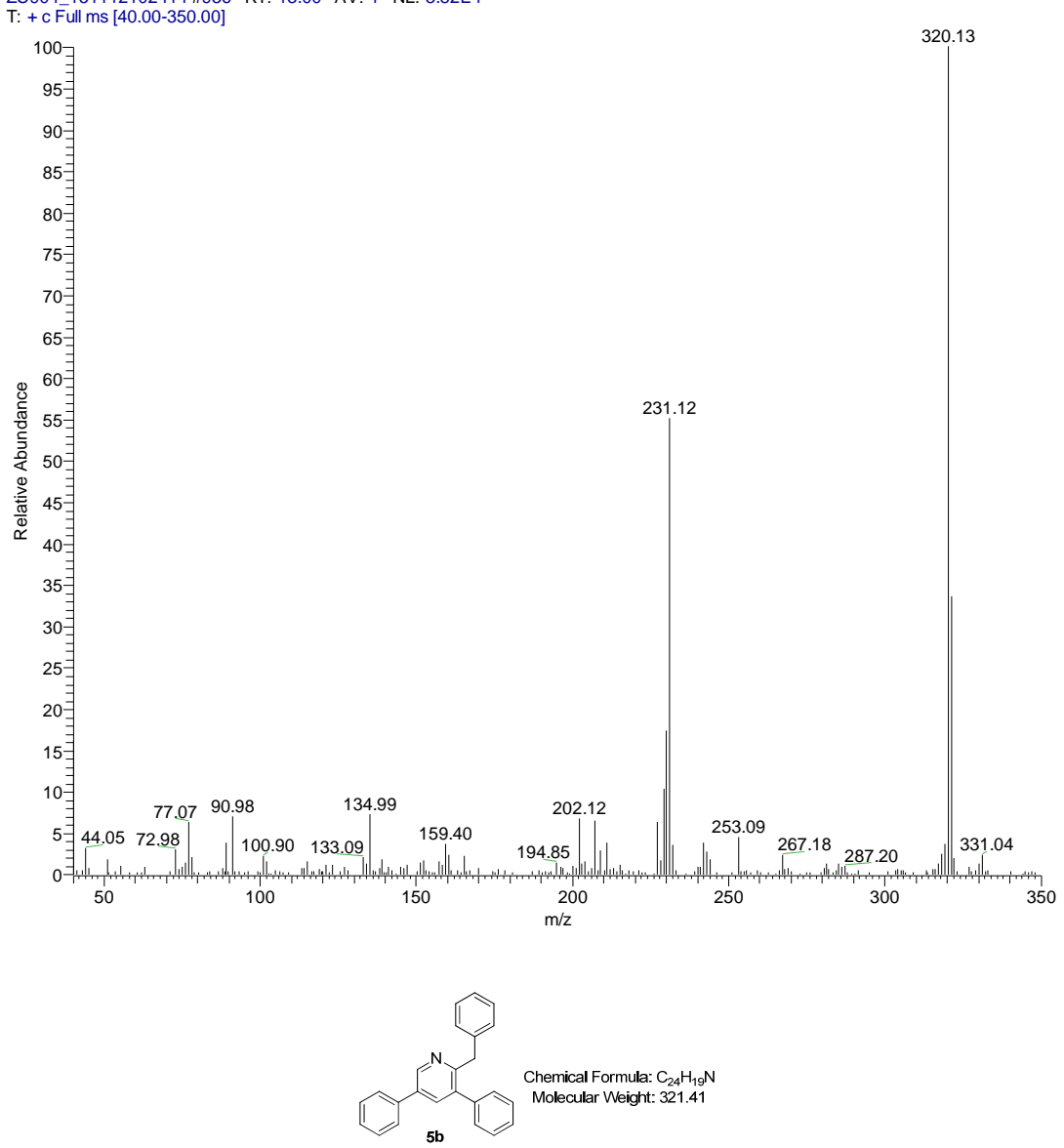


\section{Characterization data for compound 3 and 5}

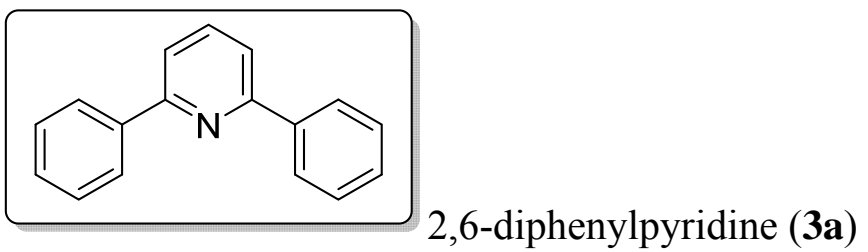

White crystalline solid (168 mg, 73\%); mp $=75-78{ }^{\circ} \mathrm{C} ;{ }^{1} \mathrm{H}$ NMR $\left(600 \mathrm{MHz}, \mathrm{CDCl}_{3}\right) \delta$ $8.15(\mathrm{~d}, J=7.8 \mathrm{~Hz}, 4 \mathrm{H}), 7.79(\mathrm{t}, J=7.8 \mathrm{~Hz}, 1 \mathrm{H}), 7.67$ (d, $J=7.8 \mathrm{~Hz}, 2 \mathrm{H}), 7.49$ (t, $J=$ $7.8 \mathrm{~Hz}, 4 \mathrm{H}), 7.42(\mathrm{~m}, 2 \mathrm{H}) .{ }^{13} \mathrm{C} \mathrm{NMR}\left(150 \mathrm{MHz}, \mathrm{CDCl}_{3}\right) \delta 156.74,139.36,137.48$, $128.95,128.64,126.95,118.63$. IR (KBr): 1633.06, 1588.22, 1563.08, 1489.43, 1453.57, 1439.52, 1385.05, 1313.55, 1269.34, 1167.42, 1099.16, 1074.08, 1016.51. 987.82, 925.66, 823.82, 776.29, 757.95. HRMS (ESI): $\mathrm{m} / \mathrm{z}[\mathrm{M}+\mathrm{H}]^{+}$calcd for $\mathrm{C}_{17} \mathrm{H}_{14} \mathrm{~N}$ : 232.1124; found: 232.1121 .

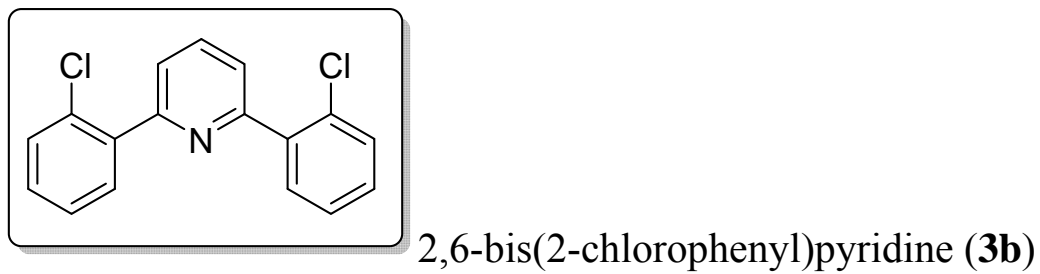

White solid (224 mg, 75\%); $\mathrm{mp}=151-154{ }^{\circ} \mathrm{C} ;{ }^{1} \mathrm{H} \mathrm{NMR}\left(600 \mathrm{MHz}, \mathrm{CDCl}_{3}\right) \delta 8.08(\mathrm{~d}$, $J=8.4 \mathrm{~Hz}, 4 \mathrm{H}), 7.82(\mathrm{t}, J=7.8 \mathrm{~Hz}, 1 \mathrm{H}), 7.67(\mathrm{~d}, J=7.8 \mathrm{~Hz}, 2 \mathrm{H}), 7.47(\mathrm{~d}, J=8.4 \mathrm{~Hz}$, $4 \mathrm{H}) .{ }^{13} \mathrm{C} \mathrm{NMR}\left(150 \mathrm{MHz}, \mathrm{CDCl}_{3}\right) \delta 155.69,137.75,137.63,135.19,128.88,128.20$, 118.65. IR (KBr): 1637.56, 1384.55, 1184.77, 1126.17. 800.62, 688.15, 618.63, 480.50. HRMS (ESI): $\mathrm{m} / \mathrm{z}[\mathrm{M}+\mathrm{H}]^{+}$calcd for $\mathrm{C}_{17} \mathrm{H}_{12} \mathrm{NCl}_{2}$ : 300.0341 ; found: 300.0342 .

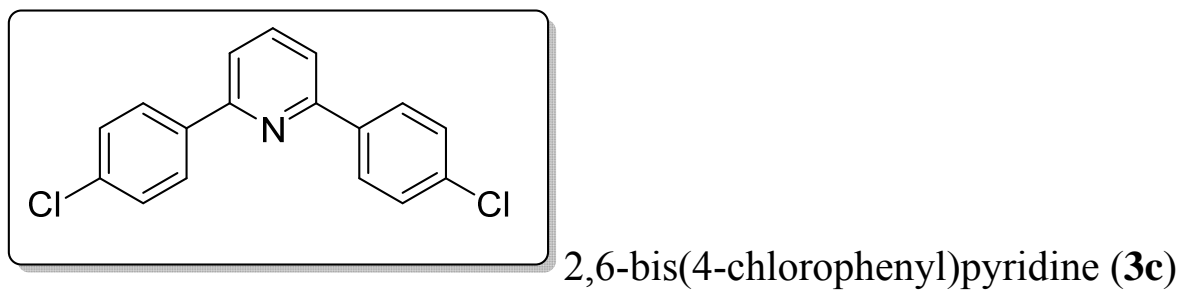

White crystalline solid (240 mg, 80\%); mp $=157-160{ }^{\circ} \mathrm{C} ;{ }^{1} \mathrm{H} \mathrm{NMR}\left(600 \mathrm{MHz}, \mathrm{CDCl}_{3}\right)$ $\delta 8.08(\mathrm{~d}, J=8.4 \mathrm{~Hz}, 4 \mathrm{H}), 7.83(\mathrm{~d}, J=7.8 \mathrm{~Hz}, 1 \mathrm{H}), 7.68(\mathrm{~d}, J=7.8 \mathrm{~Hz}, 2 \mathrm{H}), 7.47(\mathrm{~d}$, $J=8.4 \mathrm{~Hz}, 4 \mathrm{H}) .{ }^{13} \mathrm{C}$ NMR $\left(150 \mathrm{MHz}, \mathrm{CDCl}_{3}\right) \delta 155.69,137.82,137.56,135.23$, 128.89, 128.23, 118.70. IR (KBr): 1637.63, 1384.17, 1301.52, 1184.74, 1126.65, 1012.62. 799.85, 688.18, 619.38, 482.86. HRMS (ESI): $\mathrm{m} / \mathrm{z}[\mathrm{M}+\mathrm{H}]^{+}$calcd for $\mathrm{C}_{17} \mathrm{H}_{12} \mathrm{NCl}_{2}$ : 300.0341 ; found: 300.0343 . 


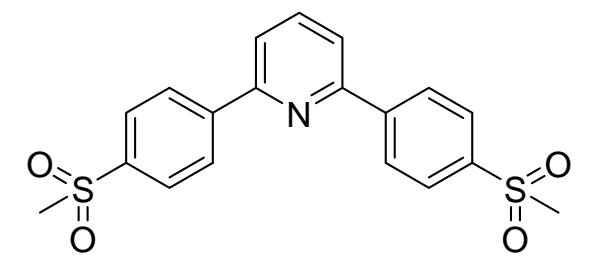

2,6-bis(4-(methylsulfonyl)phenyl)pyridine

(3d)

Pale yellow solid (321 mg, 83\%); mp $=254-257{ }^{\circ} \mathrm{C} ;{ }^{1} \mathrm{H}$ NMR $\left(600 \mathrm{MHz}, \mathrm{CDCl}_{3}\right) \delta$ $8.33(\mathrm{~d}, J=7.8 \mathrm{~Hz}, 4 \mathrm{H}), 8.09$ (d, $J=8.4 \mathrm{~Hz}, 4 \mathrm{H}), 7.97$ (t, $J=7.8 \mathrm{~Hz}, 1 \mathrm{H}), 7.86$ (d, $J=$ $7.8 \mathrm{~Hz}, 2 \mathrm{H}), 3.12(\mathrm{~s}, 6 \mathrm{H}) .{ }^{13} \mathrm{C}$ NMR $\left(150 \mathrm{MHz}, \mathrm{DMSO}-d_{6}\right) \delta 154.19,142.90,141.05$, 138.98, 127.51, 127.46 120.93, 43.41. IR (KBr): 1632.64, 1587.53, 1562.28, 1450.51, 1302.46, 1191.10, 1144.39, 1096.72, 1014.12. 969.92, 799.32, 766.03, 684.78, 648.38, 557.34, 537.38. HRMS (ESI): $\mathrm{m} / \mathrm{z}[\mathrm{M}+\mathrm{H}]^{+}$calcd for $\mathrm{C}_{19} \mathrm{H}_{18} \mathrm{O}_{4} \mathrm{NS}_{2}$ : 388.0671 ; found: 388.0672 .

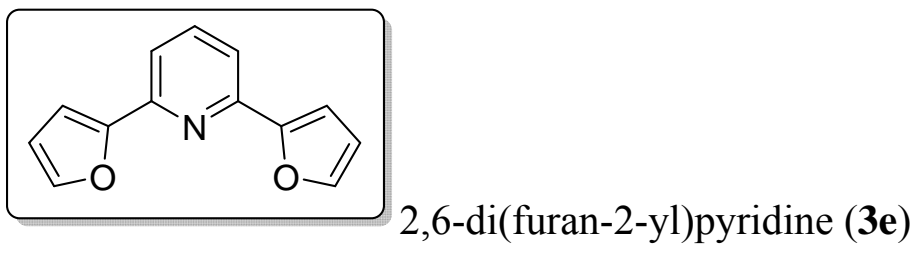

Yellow crystalline solid (152 mg, 72\%); mp $=79-81{ }^{\circ} \mathrm{C} ;{ }^{1} \mathrm{H}$ NMR $\left(600 \mathrm{MHz}, \mathrm{CDCl}_{3}\right)$ $\delta 7.74(\mathrm{~d}, J=7.8 \mathrm{~Hz}, 1 \mathrm{H}), 7.62-7.48(\mathrm{~m}, 4 \mathrm{H}), 7.18(\mathrm{~m}, 2 \mathrm{H}), 6.54(\mathrm{~m}, 2 \mathrm{H}) .{ }^{13} \mathrm{C} \mathrm{NMR}$ $\left.\left(150 \mathrm{MHz}, \mathrm{CDCl}_{3}\right) \delta 153.45\right), 149.00,143.33,137.32,116.72,112.02,109.10 . \mathrm{IR}$ (KBr): 2422.24, 2375.92, 2342.24, 1838.06, 1647.83, 1569.62, 1513.32, 1464.16, 1426.81, 1191.27, 1017.10. 500.62, 467.45, 438.27. HRMS (ESI): $\mathrm{m} / \mathrm{z}[\mathrm{M}+\mathrm{H}]^{+}$calcd for $\mathrm{C}_{13} \mathrm{H}_{10} \mathrm{O}_{2} \mathrm{~N}$ : 212.0709; found: 212.0706.

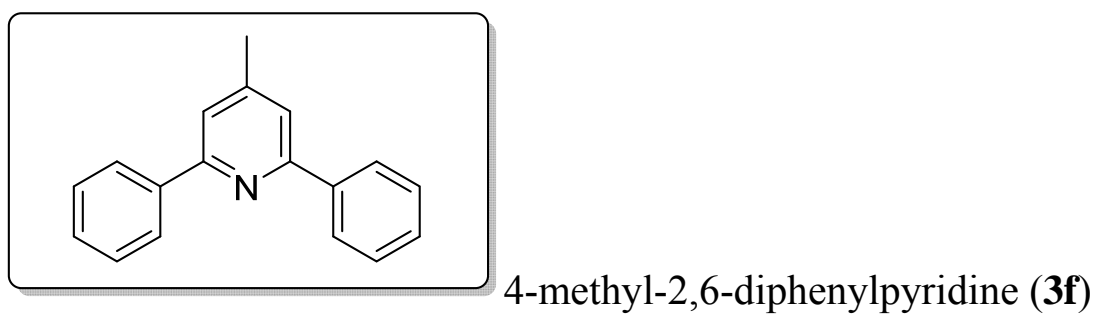

White solid (132 mg, 54\%); $\mathrm{mp}=56-59{ }^{\circ} \mathrm{C} ;{ }^{1} \mathrm{H}$ NMR $\left(600 \mathrm{MHz}, \mathrm{CDCl}_{3}\right) \delta 8.13(\mathrm{~d}, J$ $=7.8 \mathrm{~Hz}, 4 \mathrm{H}), 7.48(\mathrm{~m}, 6 \mathrm{H}), 7.43-7.39(\mathrm{~m}, 2 \mathrm{H}), 2.44(\mathrm{~s}, 3 \mathrm{H}) .{ }^{13} \mathrm{C}$ NMR $(150 \mathrm{MHz}$, $\left.\mathrm{CDCl}_{3}\right) \delta 156.69,148.32,139.47,128.80,128.57,126.98,119.72,21.42 . \mathrm{IR}(\mathrm{KBr})$ : $1632.18,1605.46,1555.51,1493.77,1408.06,1247.12,1027.20$. 918.71, 869.17, 768.13, 736.54, 694.98, 629.68, 538.39. 921.86, 861.97, 778.13, 741.65, 697.31, 631.82, 563.18. HRMS (ESI): $\mathrm{m} / \mathrm{z}[\mathrm{M}+\mathrm{H}]^{+}$calcd for $\mathrm{C}_{18} \mathrm{H}_{16} \mathrm{~N}: 246.1281$; found: 246.1277. 


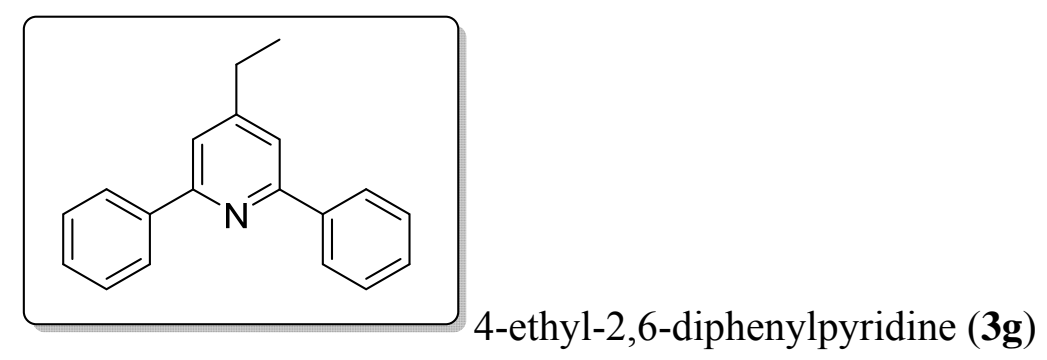

White solid (147 mg, 57\%); $\mathrm{mp}=84-86{ }^{\circ} \mathrm{C} ;{ }^{1} \mathrm{H}$ NMR $\left(600 \mathrm{MHz}, \mathrm{CDCl}_{3}\right) \delta 8.14(\mathrm{~d}, J$ $=7.2 \mathrm{~Hz}, 4 \mathrm{H}), 7.54(\mathrm{~s}, 2 \mathrm{H}), 7.49(\mathrm{t}, J=7.2 \mathrm{~Hz}, 4 \mathrm{H}), 7.42(\mathrm{t}, J=7.2 \mathrm{~Hz}, 2 \mathrm{H}), 2.78(\mathrm{q}$, $J=7.2 \mathrm{~Hz}, 2 \mathrm{H}), 1.35(\mathrm{t}, J=7.2 \mathrm{~Hz}, 3 \mathrm{H}) .{ }^{13} \mathrm{C} \mathrm{NMR}\left(150 \mathrm{MHz}, \mathrm{CDCl}_{3}\right) \delta 156.88$, 154.38, 139.66, 128.79, 128.59, 127.03, 118.57, 28.69, 14.67. IR (KBr): 918.72, 869.17, 768.13, 736.54, 694.98, 629.68, 538.39. HRMS (ESI): $\mathrm{m} / \mathrm{z}[\mathrm{M}+\mathrm{H}]^{+}$calcd for $\mathrm{C}_{19} \mathrm{H}_{18} \mathrm{~N}: 260.1437$; found: 260.1434 .

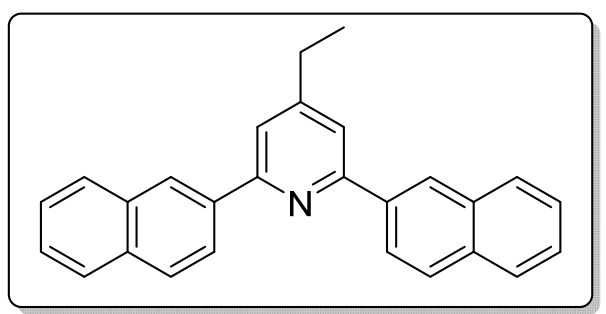

4-ethyl-2,6-di(naphthalen-2-yl)pyridine (3h)

White solid (161 mg, 45\%); mp $=227-229{ }^{\circ} \mathrm{C} ;{ }^{1} \mathrm{H} \mathrm{NMR}\left(600 \mathrm{MHz}, \mathrm{CDCl}_{3}\right) \delta 8.64$ (s, $2 \mathrm{H}), 8.37(\mathrm{~d}, J=8.4 \mathrm{~Hz}, 2 \mathrm{H}), 8.00(\mathrm{~m}, 4 \mathrm{H}), 7.90(\mathrm{~d}, J=7.8 \mathrm{~Hz}, 2 \mathrm{H}), 7.72(\mathrm{~s}, 2 \mathrm{H})$, $7.52(\mathrm{~m}, 4 \mathrm{H}), 2.85(\mathrm{q}, J=7.8 \mathrm{~Hz}, 2 \mathrm{H}), 1.42(\mathrm{t}, J=7.8 \mathrm{~Hz}, 3 \mathrm{H}) .{ }^{13} \mathrm{C}$ NMR $(150 \mathrm{MHz}$, $\left.\mathrm{CDCl}_{3}\right) \delta 156.91,136.98,133.67,133.51,128.72$ (s), 128.30 (s), 127.68 (s), 126.38, 126.18, 124.96, 119.00, 28.80, 14.76. IR (KBr): 1638, 1556, 1415, 1184, 1129, 857, 823, 765, 623, 477. HRMS (ESI): $\mathrm{m} / \mathrm{z}[\mathrm{M}+\mathrm{H}]^{+}$calcd for $\mathrm{C}_{27} \mathrm{H}_{22} \mathrm{~N}: 360.1749$; found: 360.1749 .<smiles>CCC(C)Cc1cc(-c2ccccc2)nc(-c2ccccc2)c1</smiles>

4-isobutyl-2,6-diphenylpyridine (3i)

Yellow oil (115 mg, 40\%); ${ }^{1} \mathrm{H}$ NMR $\left(600 \mathrm{MHz}, \mathrm{CDCl}_{3}\right) \delta 8.14(\mathrm{~d}, J=7.2 \mathrm{~Hz}, 4 \mathrm{H})$, $7.48(\mathrm{~m}, 6 \mathrm{H}), 7.41(\mathrm{~m}, 2 \mathrm{H}), 2.59(\mathrm{~d}, J=7.2 \mathrm{~Hz}, 2 \mathrm{H}), 2.00(\mathrm{~m}, 1 \mathrm{H}), 0.97(\mathrm{~d}, J=6.6 \mathrm{~Hz}$, $6 \mathrm{H}) .{ }^{13} \mathrm{C} \mathrm{NMR}\left(150 \mathrm{MHz}, \mathrm{CDCl}_{3}\right) \delta 156.68,151.88,139.66,128.77,128.58,127.00$, 119.67, 45.13, 29.74, 22.38. IR (KBr): 2956, 1637, 1416, 1385, 1192, 1136, 775, 739, 694, 632, 480. HRMS (ESI): $\mathrm{m} / \mathrm{z}[\mathrm{M}+\mathrm{H}]^{+}$calcd for $\mathrm{C}_{21} \mathrm{H}_{22} \mathrm{~N}$ : 288.1749; found: 288.1747 . 


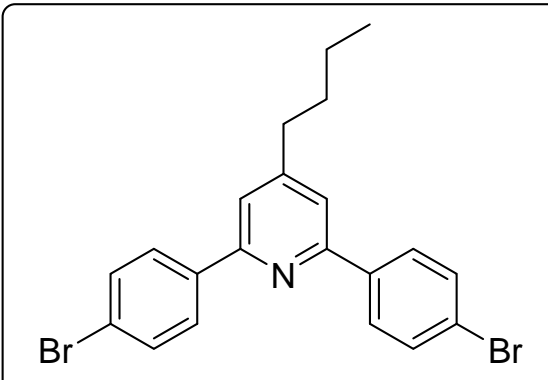

2,6-bis(4-bromophenyl)-4-butylpyridine (3j)

Yellow crystalline solid $(279 \mathrm{mg}, 63 \%) ; \mathrm{mp}=133-136{ }^{\circ} \mathrm{C} ;{ }^{1} \mathrm{H}$ NMR $(600 \mathrm{MHz}$, $\left.\mathrm{CDCl}_{3}\right) \delta 8.04-7.93(\mathrm{~m}, 4 \mathrm{H}), 7.60(\mathrm{~d}, J=7.2 \mathrm{~Hz}, 4 \mathrm{H}), 7.48(\mathrm{~s}, 2 \mathrm{H}), 2.71(\mathrm{t}, J=7.8 \mathrm{~Hz}$, 2H), 1.72-1.64 (m, 2H), 1.47-1.35 (m, 2H), $0.966(\mathrm{t}, J=7.8 \mathrm{~Hz}, 3 \mathrm{H}) .{ }^{13} \mathrm{C}$ NMR $(150$ $\left.\mathrm{MHz}, \mathrm{CDCl}_{3}\right) \delta 155.69,153.59,138.24,131.72,128.54,123.34,119.09,35.43,32.69$, 22.38, 13.91. IR (KBr): 2957.71, 2927.88, 2856.87, 1687.26, 1601.16, 1547.95, 1484.97, 1418.01, 1386.49, 1261.16, 1173.94, 1097.79, 1069.16, 1007.69, 814.81, 743.42, 639.89, 551.49. HRMS (ESI): $\mathrm{m} / \mathrm{z}[\mathrm{M}+\mathrm{H}]^{+}$calcd for $\mathrm{C}_{21} \mathrm{H}_{19} \mathrm{Br}_{2} \mathrm{~N}: 443.9957$; found: 443.9957.

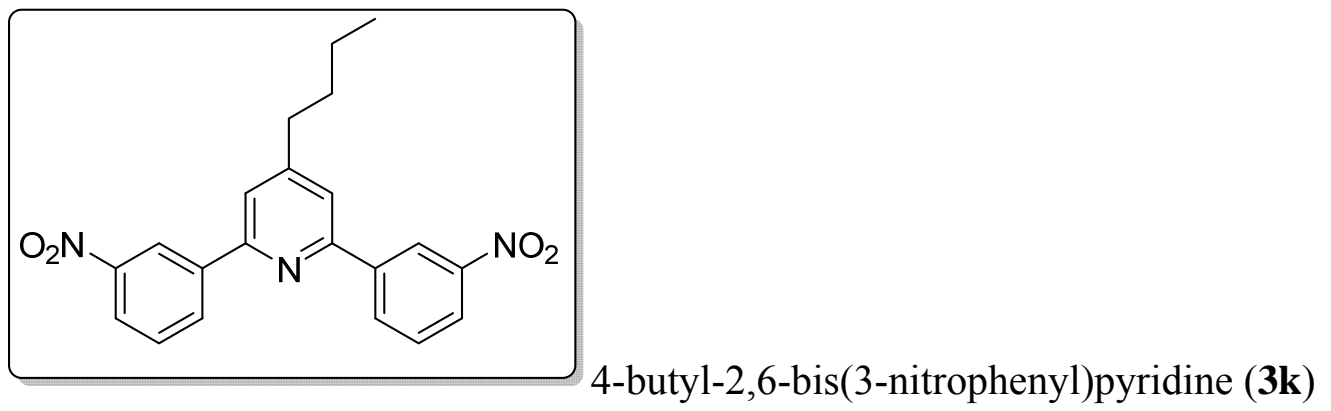

Brown oil (282 mg, 75\%); ${ }^{1} \mathrm{H}$ NMR (600 MHz, $\left.\mathrm{CDCl}_{3}\right) \delta 8.93(\mathrm{~s}, 2 \mathrm{H}), 8.54(\mathrm{~d}, J=$ $7.8 \mathrm{~Hz}, 2 \mathrm{H}), 8.29(\mathrm{~m}, 2 \mathrm{H}), 7.69(\mathrm{~m}, 4 \mathrm{H}), 2.81(\mathrm{t}, J=7.8 \mathrm{~Hz}, 2 \mathrm{H}), 1.80-1.72(\mathrm{~m}, 2 \mathrm{H})$, $1.45-1.50(\mathrm{~m}, 2 \mathrm{H}), 1.00(\mathrm{t}, J=7.8 \mathrm{~Hz}, 3 \mathrm{H}) .{ }^{13} \mathrm{C} \mathrm{NMR}\left(150 \mathrm{MHz}, \mathrm{CDCl}_{3}\right) \delta 154.51$, 148.62, 140.67, 132.93, 129.70, 123.68, 121.67, 120.21, 35.43, 32.71, 22.41, 13.87 . IR (KBr): 2929.81, 1636.89, 1530.12, 1384.17, 1349.31, 1184.51, 1150.24, 1126.27, 1044.55, 1015.08, 814.57, 687.04, 619.03, 568.29. HRMS (ESI): $\mathrm{m} / \mathrm{z}[\mathrm{M}+\mathrm{H}]^{+}$calcd for $\mathrm{C}_{21} \mathrm{H}_{20} \mathrm{O}_{4} \mathrm{~N}_{3}: 378.1451$; found: 378.1448 . 


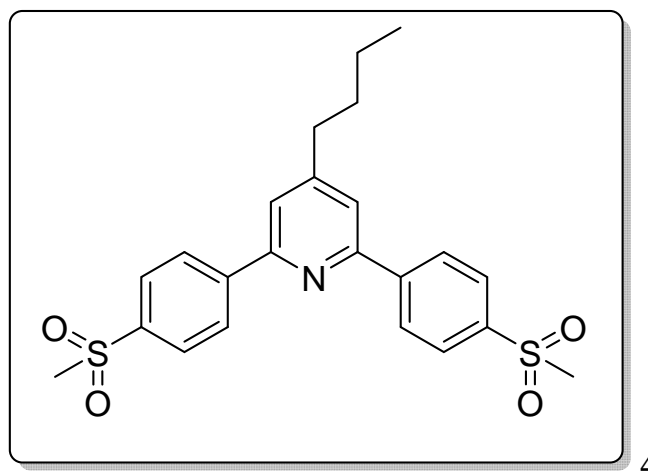

ne (3I)

Yellow solid (328 mg, 74\%); $\mathrm{mp}=225-227{ }^{\circ} \mathrm{C} ;{ }^{1} \mathrm{H}$ NMR $\left(600 \mathrm{MHz}, \mathrm{CDCl}_{3}\right) \delta 8.32$ $(\mathrm{d}, J=8.4 \mathrm{~Hz}, 4 \mathrm{H}), 8.08(\mathrm{~d}, J=8.4 \mathrm{~Hz}, 4 \mathrm{H}), 7.67(\mathrm{~s}, 2 \mathrm{H}), 3.12(\mathrm{~s}, 6 \mathrm{H}), 2.80$ (t, $J=7.8$ $\mathrm{Hz}, 2 \mathrm{H}), 1.72-1.77(\mathrm{~m}, 2 \mathrm{H}), 1.43-1.47(\mathrm{~m}, 2 \mathrm{H}), 0.99(\mathrm{t}, J=7.8 \mathrm{~Hz}, 3 \mathrm{H}) .{ }^{13} \mathrm{C} \mathrm{NMR}$ $\left(150 \mathrm{MHz}, \mathrm{CDCl}_{3}\right) \delta 155.11,154.35,144.25,140.56,127.91,127.79,120.90,44.55$ (s), 35.41, 32.66, 22.36, 13.86. IR (KBr): 3002.79, 2924.65, 1597.82, 1551.22, 1428.80, 1305.28, 1151.91, 1087.07, 1013.17, 966.1913, 949.97, 844.14, 767.35, 687.78, 621.52, 562.97, 543.56, 524.28. HRMS (ESI): $\mathrm{m} / \mathrm{z}[\mathrm{M}+\mathrm{H}]^{+}$calcd for $\mathrm{C}_{23} \mathrm{H}_{26} \mathrm{O}_{4} \mathrm{NS}_{2}$ : 444.1301; found: 444.1298.

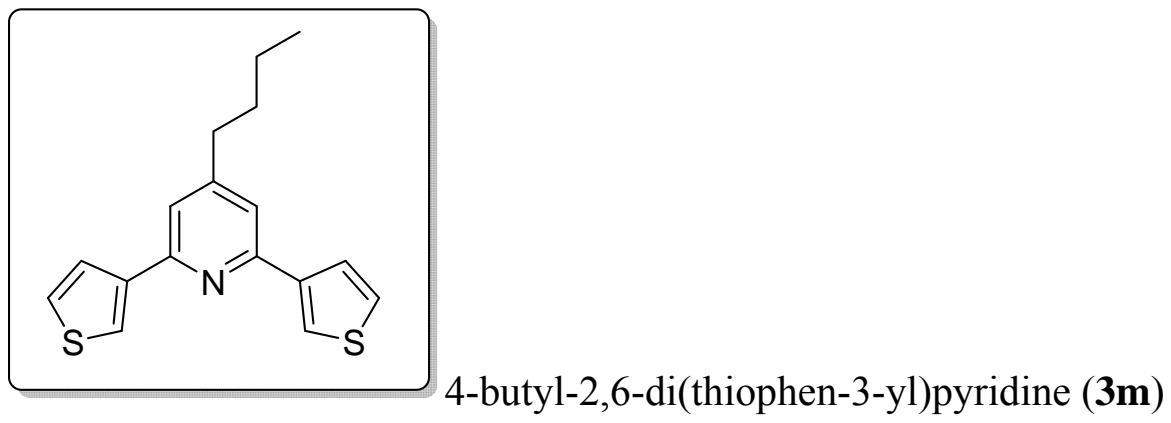

White solid (194 mg, 65\%); mp = 153-156 ${ }^{\circ} \mathrm{C} ;{ }^{1} \mathrm{H}$ NMR $\left(600 \mathrm{MHz}, \mathrm{CDCl}_{3}\right) \delta 7.99(\mathrm{~d}$, $J=8.4 \mathrm{~Hz}, 4 \mathrm{H}), 7.61(\mathrm{~d}, J=8.4 \mathrm{~Hz}, 4 \mathrm{H}), 7.49$ (s, 2H), 2.72 (t, $J=7.8 \mathrm{~Hz}, 2 \mathrm{H})$, 1.67-1.71 (m, 2H), 1.40-1.44 (m, 2H), $0.97(\mathrm{t}, J=7.2 \mathrm{~Hz}, 3 \mathrm{H}) .{ }^{13} \mathrm{C}$ NMR (150 MHz, $\left.\mathrm{CDCl}_{3}\right) \delta 153.07,152.93,142.48,126.40,125.94,123.39,118.57,35.29,32.61$, 22.34, 13.92. IR (KBr): 1637.57, 1384.91, 1302.19, 1184.99, 1126.45, 1012.50, 816.77, 688.25, 619.54, 479.43. MS: $\mathrm{m} / \mathrm{z}$ [M] calcd for $\mathrm{C}_{17} \mathrm{H}_{17} \mathrm{NS}_{2}$, found: 299.14 . 


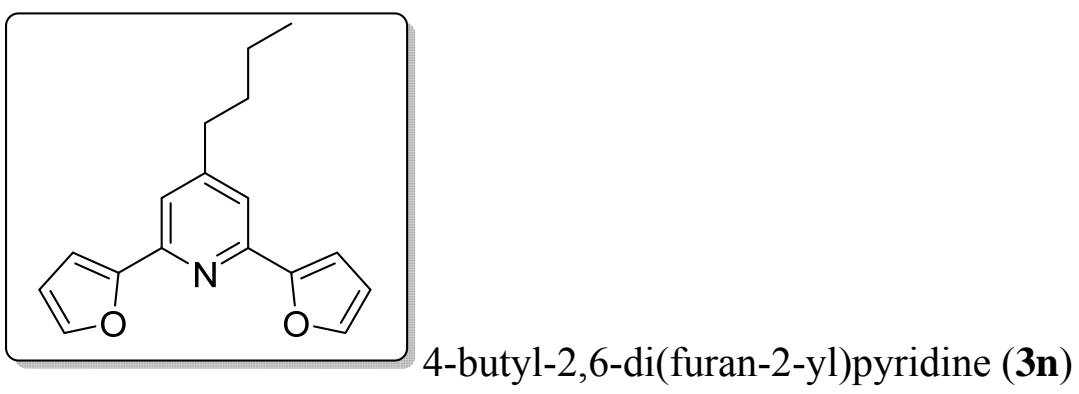

Yellow oil (187 mg, 70\%); ${ }^{1} \mathrm{H}$ NMR (600 MHz, $\left.\mathrm{CDCl}_{3}\right) \delta 7.53(\mathrm{~s}, 2 \mathrm{H}), 7.41(\mathrm{~s}, 2 \mathrm{H})$, 7.125-7.13 (m, 2H), 2.7-2.64 (m, 2H), 1.71-1.66 (m, 2H), 1.42-1.38 (m, 2H), $0.95(\mathrm{t}, J$ $=7.2 \mathrm{~Hz}, 3 \mathrm{H}) .{ }^{13} \mathrm{C} \mathrm{NMR}\left(150 \mathrm{MHz}, \mathrm{CDCl}_{3}\right) \delta 153.81,153.08,149.04,143.04,117.02$, 111.94, 108.71, 35.28, 32.42, 22.32, 13.90. IR (KBr): 2961.06, 2865.77, 1616.48, 1556.93, 1494.90, 1404.31, 1261.19, 1221.72, 1097.85, 1015.55, 865.12, 802.13, 738.49, 688.99, 594.73, 476.09. HRMS (ESI): $\mathrm{m} / \mathrm{z}[\mathrm{M}+\mathrm{H}]^{+}$calcd for $\mathrm{C}_{17} \mathrm{H}_{18} \mathrm{O}_{2} \mathrm{~N}$ : 268.1337; found: 268.1332 .

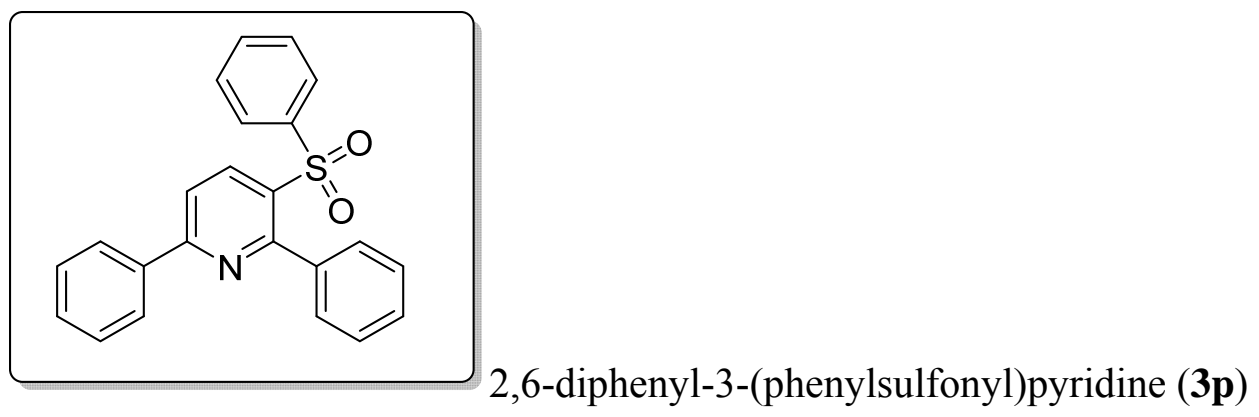

White solid (274 mg, 74\%); $\mathrm{mp}=170-171^{\circ} \mathrm{C} ;{ }^{1} \mathrm{H}$ NMR $\left(600 \mathrm{MHz}, \mathrm{CDCl}_{3}\right) \delta 8.75(\mathrm{~d}$, $J=8.4 \mathrm{~Hz}, 1 \mathrm{H}), 8.05-8.06(\mathrm{~m}, 2 \mathrm{H}), 7.92(\mathrm{~d}, J=8.4 \mathrm{~Hz}, 1 \mathrm{H}), 7.43-7.44(\mathrm{~m}, 3 \mathrm{H})$, 7.36-7.41 (m, 2H), 7.22-7.27 (m, 6H), 7.18-7.21 (m, 2H). ${ }^{13} \mathrm{C}$ NMR (150 MHz, $\left.\mathrm{CDCl}_{3}\right) \delta 159.98,158.72,139.92,138.13,137.93,137.23,135.16,132.83,130.27$, 129.71, 128.80, 128.61, 128.37, 127.72, 127.50, 127.39, 118.43. IR (KBr): 1632.55, $1568.41,1494.59,1445.29,1425.31,1376.81,1315.97,1160.37,1146.64,1113.25$, 1084.33, 1016.64, 838.10, 771.86, 762.26, 728.13, 701.82, 685.06. HRMS (ESI): m/z $[\mathrm{M}+\mathrm{H}]^{+}$calcd for $\mathrm{C}_{23} \mathrm{H}_{18} \mathrm{O}_{2} \mathrm{NS}: 372.1055$; found: 372.1053 .

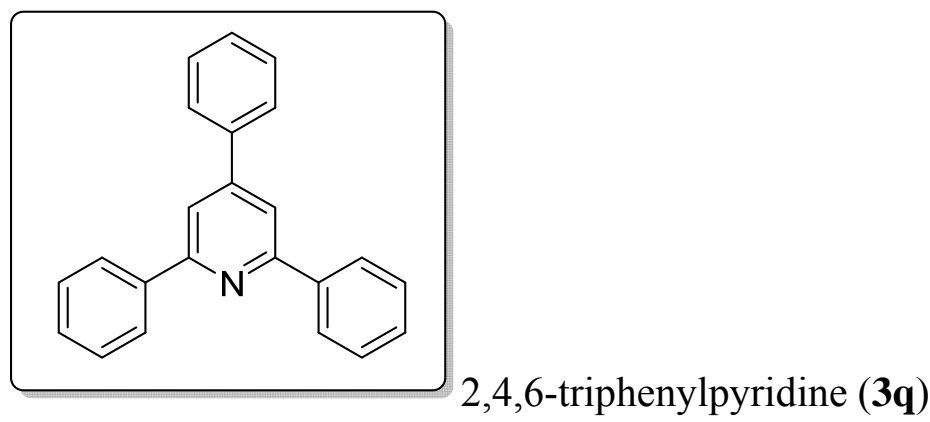


Pale yellow solid $(261 \mathrm{mg}, 85 \%) ; \mathrm{mp}=136-138{ }^{\circ} \mathrm{C} ;{ }^{1} \mathrm{H}$ NMR $\left(300 \mathrm{MHz}, \mathrm{CDCl}_{3}\right) \delta$ 8.27-8.15 (m, 4H), 7.89 (s, 2H), 7.73-7.77 (m, 2H), 7.45-7.55(m, 9H). ${ }^{13} \mathrm{C}$ NMR (75 $\left.\mathrm{MHz}, \mathrm{CDCl}_{3}\right) \delta 157.46,150.27,139.44,139.00,129.11,129.08,129.00,128.70$, 127.18, 127.16, 117.18. IR (KBr): 1604.8217, 1594.08, 1577.98, 1550.14, 1493.32, 1446.48, 1399.63, 1072.97, 1029.93, 869.90, 758.63, 738.25, 691.96, 634.06, 610.85, 575.69. HRMS (ESI): m/z [M+H] $]^{+}$calcd for $\mathrm{C}_{23} \mathrm{H}_{18} \mathrm{~N}$ : 308.1435 ; found: 308.1434 .

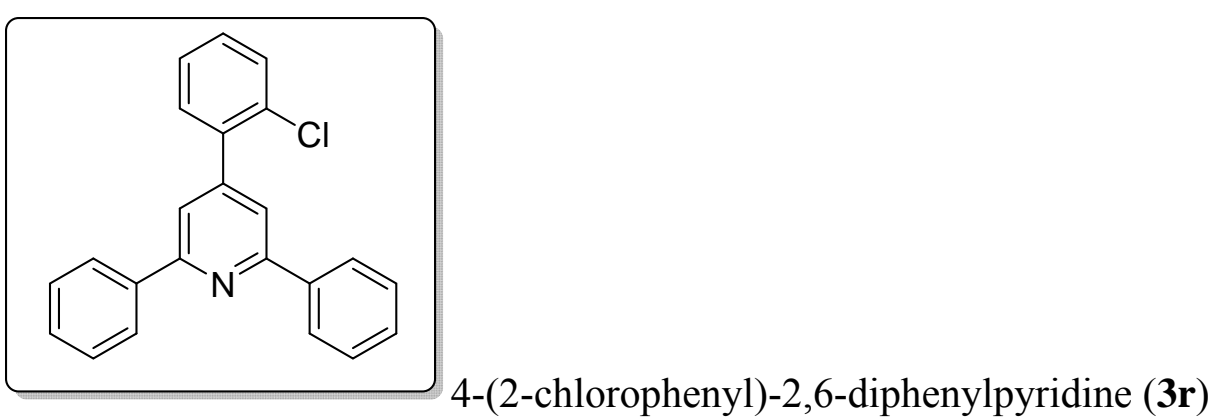

Yellow oil (266 mg, 78\%); ${ }^{1} \mathrm{H}$ NMR $\left(600 \mathrm{MHz}, \mathrm{CDCl}_{3}\right) \delta 8.19(\mathrm{~d}, J=7.8 \mathrm{~Hz}, 4 \mathrm{H})$, $7.78(\mathrm{~s}, 2 \mathrm{H}), 7.56-7.53(\mathrm{~m}, 1 \mathrm{H}), 7.49-7.52(\mathrm{~m}, 4 \mathrm{H}), 7.42-7.45(\mathrm{~m}, 3 \mathrm{H}), 7.40-7.36(\mathrm{~m}$, 2H). ${ }^{13} \mathrm{C}$ NMR $\left(150 \mathrm{MHz}, \mathrm{CDCl}_{3}\right) \delta 156.81,148.62,139.20,138.40,132.24,130.87$, 130.26, 129.69, 129.10, 128.68, 127.12, 119.51. IR (KBr): 3060.14, 1684.99, 1602.66, 1544.21, 1475.48, 1443.16, 1397.74, 1262.86, 1033.15, 880.37, 804.19, 755.84, 690.72, 629.38. HRMS (ESI): $\mathrm{m} / \mathrm{z}[\mathrm{M}+\mathrm{H}]^{+}$calcd for $\mathrm{C}_{23} \mathrm{H}_{17} \mathrm{NCl}$ : 342.1048; found: 342.1044 .

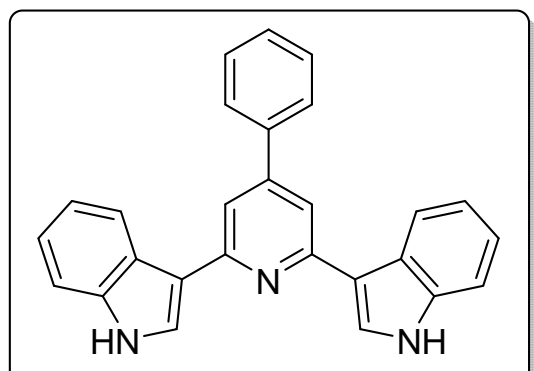

3,3'-(4-phenylpyridine-2,6-diyl)bis(1H-indole) (3s)

Yellow oil (273 mg, 71\%); ${ }^{1} \mathrm{H}$ NMR (600 MHz, DMSO- $\left.d_{6}\right) \delta 11.59$ (s, 2H), 8.60 (d, $J$ $=7.8 \mathrm{~Hz}, 2 \mathrm{H}), 8.30(\mathrm{~s}, 2 \mathrm{H}), 8.00(\mathrm{~d}, J=7.8 \mathrm{~Hz}, 2 \mathrm{H}), 7.89(\mathrm{~s}, 2 \mathrm{H}), 7.59(\mathrm{t}, J=7.2 \mathrm{~Hz}$, $2 \mathrm{H}), 7.50(\mathrm{~d}, J=7.8 \mathrm{~Hz}, 3 \mathrm{H}), 7.20(\mathrm{t}, J=7.8 \mathrm{~Hz}, 2 \mathrm{H}), 7.15(\mathrm{t}, J=7.8 \mathrm{~Hz}, 2 \mathrm{H}) .{ }^{13} \mathrm{C}$ NMR (150 MHz, DMSO- $\left.d_{6}\right) \delta 155.27,148.09,147.93,138.60,137.08,129.03$, $128.83,127.02,126.14,125.44,121.63,121.49,119.91,113.57,111.86 . \mathrm{IR}(\mathrm{KBr})$ : $1637.93,1385.15,1302.70,1183.99,1126.17,1044.69,1015.62,816.33,688.71$, 617.32. HRMS (ESI): m/z [M+H] $]^{+}$calcd for $\mathrm{C}_{27} \mathrm{H}_{20} \mathrm{~N}_{3}$ : 386.1652 ; found: 386.1652 . 


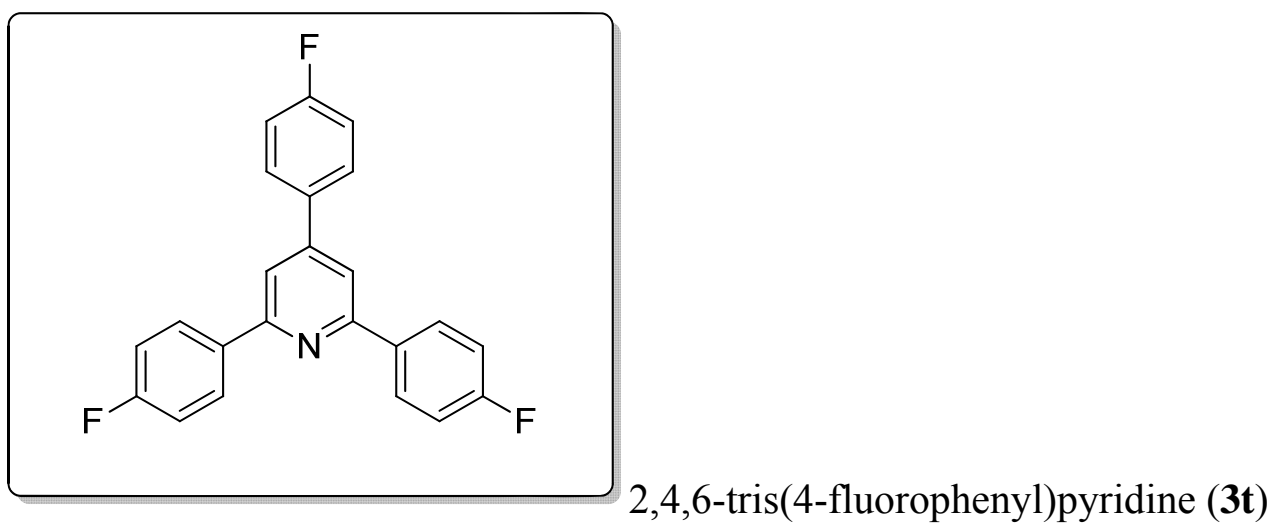

White crystalline solid (266 mg, 74\%); mp = 221-223 ${ }^{\circ} \mathrm{C} ;{ }^{1} \mathrm{H} \mathrm{NMR}\left(600 \mathrm{MHz}, \mathrm{CDCl}_{3}\right)$ $\delta$ 8.14-8.17 (m, 4H), $7.76(\mathrm{~s}, 2 \mathrm{H}), 7.68-7.70(\mathrm{~m}, 2 \mathrm{H}), 7.17-7.25(\mathrm{~m}, 6 \mathrm{H}) .{ }^{13} \mathrm{C} \mathrm{NMR}$ $\left(150 \mathrm{MHz}, \mathrm{CDCl}_{3}\right) \delta 164.49,162.84,156.53,149.40,135.39,134.90,128.89$ (d, J = $8.2 \mathrm{~Hz}), 116.77-116.52(\mathrm{~m}), 116.47,116.16(\mathrm{~d}, \mathrm{~J}=21.7 \mathrm{~Hz}), 115.71,115.57 . \mathrm{IR}$ (KBr): $1657.21,1609.47,1548.24,1511.14,1426.63,1384.49,1295.00,1223.88$, 1155.84, 1098.10, 1011.84, 821.11, 780.34, 581.27, 554.64, 515.05, 439.32. MS: m/z [M] calcd for $\mathrm{C}_{23} \mathrm{H}_{14} \mathrm{~F}_{3} \mathrm{~N}$, found: 361.22 .

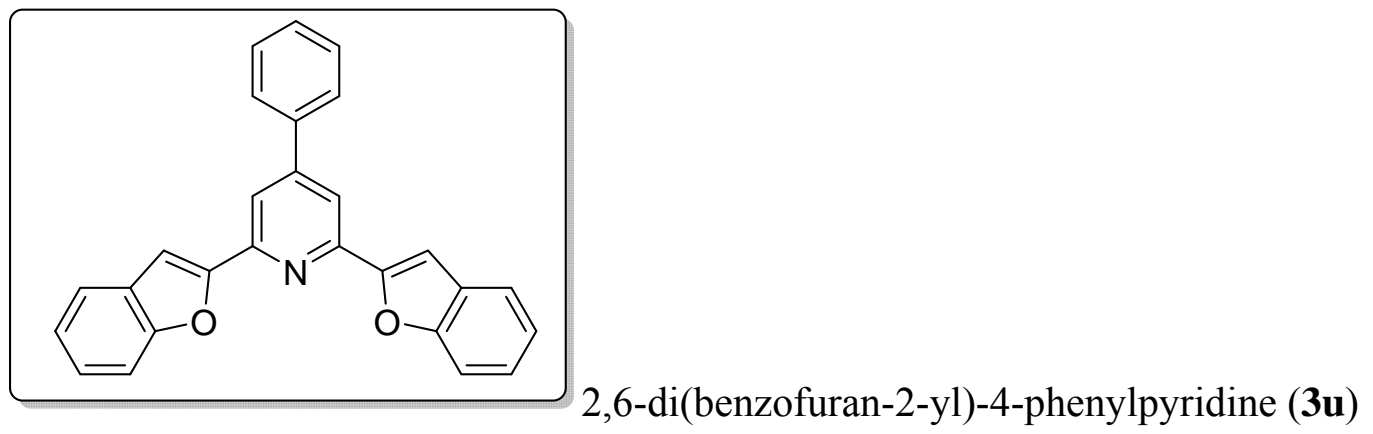

White solid (290 mg, 75\%); mp = 220-221 ${ }^{\circ} \mathrm{C} ;{ }^{1} \mathrm{H} \mathrm{NMR}\left(600 \mathrm{MHz}, \mathrm{CDCl}_{3}\right) \delta 8.11(\mathrm{~s}$, $2 \mathrm{H}), 7.86(\mathrm{~d}, J=7.2 \mathrm{~Hz}, 2 \mathrm{H}), 7.69-7.71(\mathrm{~m}, 4 \mathrm{H}), 7.55-7.62(\mathrm{~m}, 4 \mathrm{H}), 7.52(\mathrm{~m}, 1 \mathrm{H})$, $7.37(\mathrm{t}, J=7.8 \mathrm{~Hz}, 2 \mathrm{H}), 7.29(\mathrm{t}, J=7.8 \mathrm{~Hz}, 2 \mathrm{H}) .{ }^{13} \mathrm{C} \mathrm{NMR}\left(150 \mathrm{MHz}, \mathrm{CDCl}_{3}\right) \delta$ $155.45,155.34,154.83,150.32,149.64,129.45,129.17,128.84,127.15,125.32$, 123.23, 121.83, 116.81, 111.50, 105.82. IR (KBr): 1632.52, 1553.54, 1384.10, 818.13, 766.07, 750.73. HRMS (ESI): $\mathrm{m} / \mathrm{z}[\mathrm{M}+\mathrm{H}]^{+}$calcd for $\mathrm{C}_{27} \mathrm{H}_{18} \mathrm{O}_{2} \mathrm{~N}: 388.1333$; found: 388.1332 .

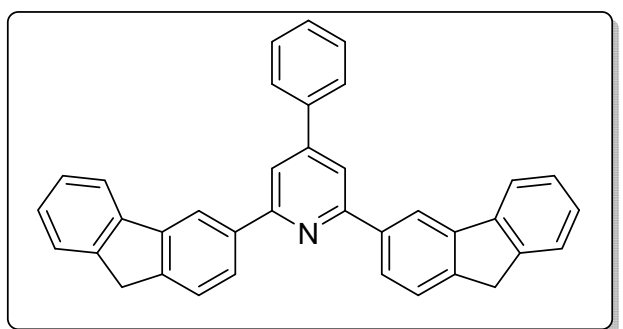

2,6-di(9H-fluoren-3-yl)-4-phenylpyridine (3v)

Pale yellow solid (352 mg, 73\%); mp $=259-261{ }^{\circ} \mathrm{C} ;{ }^{1} \mathrm{H}$ NMR $\left(600 \mathrm{MHz}, \mathrm{CDCl}_{3}\right) \delta$ 8.44 (s, 2H), 8.23 (d, $J=7.8 \mathrm{~Hz}, 2 \mathrm{H}), 7.91-7.96(\mathrm{~m}, 4 \mathrm{H}), 7.86$ (d, $J=7.8 \mathrm{~Hz}, 2 \mathrm{H})$, 
$7.80(\mathrm{~d}, J=7.8 \mathrm{~Hz}, 2 \mathrm{H}), 7.60(\mathrm{~d}, J=7.2 \mathrm{~Hz}, 2 \mathrm{H}), 7.56(\mathrm{t}, J=7.2 \mathrm{~Hz}, 2 \mathrm{H}), 7.50(\mathrm{t}, J=$ $7.2 \mathrm{~Hz}, 1 \mathrm{H}), 7.42(\mathrm{t}, J=7.8 \mathrm{~Hz}, 2 \mathrm{H}), 7.35(\mathrm{t}, J=7.8 \mathrm{~Hz}, 2 \mathrm{H}), 4.04(\mathrm{~s}, 4 \mathrm{H}) .{ }^{13} \mathrm{C} \mathrm{NMR}$ $\left(100 \mathrm{MHz}, \mathrm{CDCl}_{3}\right) \delta 157.22,143.78,143.63,142.77,141.08,138.58,136.99,129.06$, $127.19,126.95,126.72,126.25,125.01,124.01,120.18,119.94,117.34,37.19$. IR (KBr): 1592.61, 1542.79, 1450.03, 1400.59, 1300.18, 1218.87, 1075.97, 951.76, 867.60, 836.24, 760.84, 734.01, 694.47. HRMS (ESI): $\mathrm{m} / \mathrm{z}[\mathrm{M}+\mathrm{H}]^{+}$calcd for $\mathrm{C}_{37} \mathrm{H}_{26} \mathrm{~N}$ : 484.2063; found: 484.2060 .

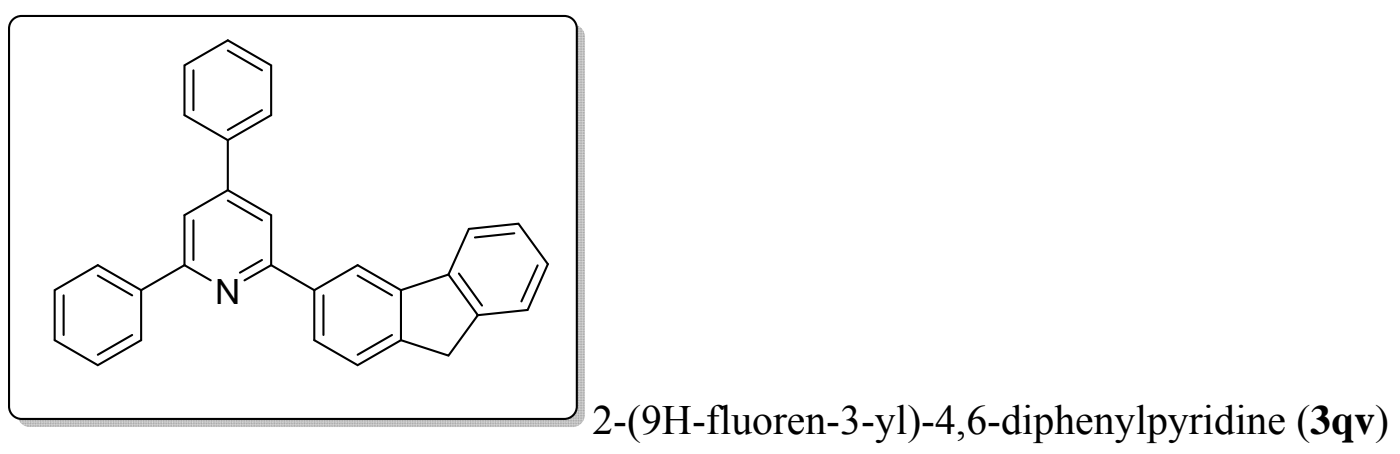

Yellow solid (142 mg, 36\%); $\mathrm{mp}=202-204{ }^{\circ} \mathrm{C} ;{ }^{1} \mathrm{H}$ NMR $\left(600 \mathrm{MHz}, \mathrm{CDCl}_{3}\right) \delta 8.41$ (s, 1H), 8.20-8.22 (m, 3H), $7.92(\mathrm{~s}, 1 \mathrm{H}), 7.90-7.85(\mathrm{~m}, 2 \mathrm{H}), 7.83(\mathrm{~d}, J=7.2 \mathrm{~Hz}, 1 \mathrm{H})$, $7.75(\mathrm{~d}, J=7.2 \mathrm{~Hz}, 2 \mathrm{H}), 7.57$ (d, $J=7.2 \mathrm{~Hz}, 1 \mathrm{H}), 7.52(\mathrm{t}, J=7.2 \mathrm{~Hz}, 4 \mathrm{H}), 7.43-7.47$ (m, 2H), 7.39 (t, $J=7.2 \mathrm{~Hz}, 1 \mathrm{H}), 7.32(\mathrm{t}, J=7.2 \mathrm{~Hz}, 1 \mathrm{H}), 3.99(\mathrm{~s}, 2 \mathrm{H}) .{ }^{13} \mathrm{C}$ NMR $(150$ $\left.\mathrm{MHz}, \mathrm{CDCl}_{3}\right) \delta 157.62,157.47,150.12,143.85,143.76,142.58,141.29,139.61$, $139.03,138.03,129.07,129.01,128.94,128.69,127.16,126.94,125.09,127.05$, 123.74, 120.20, 119.97, 117.09, 116.98, 37.03. IR (KBr): 1637.13, 1385.02, 1301.83, 1184.90, 1148.57, 1044.88, 1014.89, 967.12, 763.17, 687.85, 620.55, 564.01. HRMS (ESI): $\mathrm{m} / \mathrm{z}[\mathrm{M}+\mathrm{H}]^{+}$calcd for $\mathrm{C}_{30} \mathrm{H}_{21} \mathrm{~N}$ : 396.1747; found: 396.1753 .

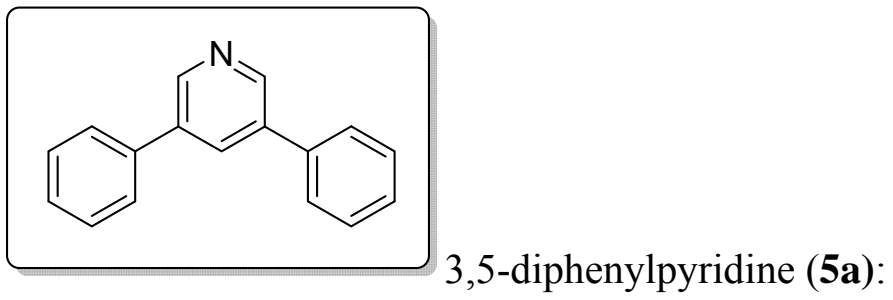

Black crystalline solid (116 mg, 75\%); mp = 132-135 ${ }^{\circ} \mathrm{C} ;{ }^{1} \mathrm{H}$ NMR $\left(600 \mathrm{MHz}, \mathrm{CDCl}_{3}\right)$ $\delta 8.83(\mathrm{~s}, 2 \mathrm{H}), 8.07(\mathrm{~s}, 1 \mathrm{H}), 7.65(\mathrm{~m}, J=7.2 \mathrm{~Hz}, 4 \mathrm{H}), 7.51$ (t, $J=7.8 \mathrm{~Hz}, 4 \mathrm{H}), 7.45(\mathrm{~m}$, 2H). ${ }^{13} \mathrm{C} \mathrm{NMR}\left(150 \mathrm{MHz}, \mathrm{CDCl}_{3}\right) \delta 146.71,137.62,136.72,133.10,129.13,128.27$, 127.24. IR (KBr): 1632.43, 1495.21, 1452.87, 1415.18, 1158.24, 1009.54, 972.64, 918.00, 892.54, 767.93, 755.91, 716.41, 698.96, 628.15, 601.17, 538.24. HRMS (ESI): $\mathrm{m} / \mathrm{z}[\mathrm{M}+\mathrm{H}]^{+}$calcd for $\mathrm{C}_{17} \mathrm{H}_{14} \mathrm{~N}$ : 232.1123; found: 232.1121 . 


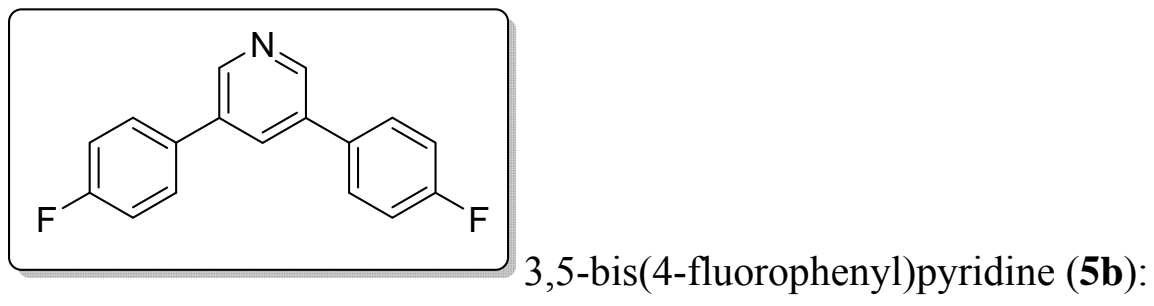

Yellow solid (128 mg, 72\%); $\mathrm{mp}=176-178{ }^{\circ} \mathrm{C} ;{ }^{1} \mathrm{H}$ NMR $\left(600 \mathrm{MHz}, \mathrm{CDCl}_{3}\right) \delta 8.77$ $(\mathrm{s}, 2 \mathrm{H}), 7.96(\mathrm{~s}, 1 \mathrm{H}), 7.60(\mathrm{~m}, 4 \mathrm{H}), 7.20(\mathrm{t}, J=8.4 \mathrm{~Hz}, 4 \mathrm{H}) \cdot{ }^{13} \mathrm{C}$ NMR $(150 \mathrm{MHz}$, $\left.\mathrm{CDCl}_{3}\right) \delta 163.82,162.17,146.67,135.78,133.64,132.68,128.91(\mathrm{~d}, J=8.0 \mathrm{~Hz})$, 116.43 - $116.35(\mathrm{~m}), 116.15(\mathrm{~d}, J=21.6 \mathrm{~Hz})$. IR (KBr): 1605.99, 1514.21, 1437.65, 1389.82, 1306.61, 1235.45, 1224.03, 1164.96, 1103.05, 1008.51, 962.26, 839.63, 780.32, 722.41, 713.17, 670.28, 567.15, 542.18. HRMS (ESI): $\mathrm{m} / \mathrm{z}[\mathrm{M}+\mathrm{H}]^{+}$calcd for $\mathrm{C}_{17} \mathrm{H}_{12} \mathrm{NF}_{2}$ : 268.0935; found: 268.0932 .

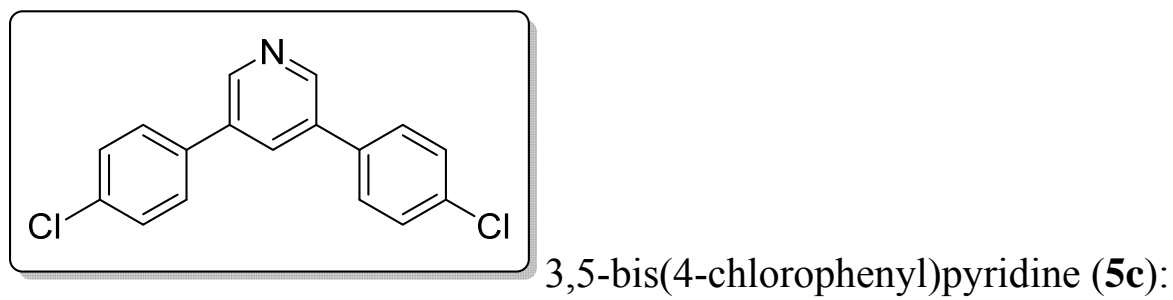

Brown solid (147 mg, 74\%); mp = 209-211 ${ }^{\circ} \mathrm{C} ;{ }^{1} \mathrm{H}$ NMR $\left(600 \mathrm{MHz}, \mathrm{CDCl}_{3}\right) \delta 8.80(\mathrm{~s}$, 2H), $7.99(\mathrm{~s}, 1 \mathrm{H}), 7.57$ (d, $J=7.8 \mathrm{~Hz}, 4 \mathrm{H}), 7.48$ (d, $J=7.8 \mathrm{~Hz}, 4 \mathrm{H}) .{ }^{13} \mathrm{C}$ NMR $(150$ $\left.\mathrm{MHz}, \mathrm{CDCl}_{3}\right) \delta 146.72,135.81,135.71,134.69,132.72,129.38,128.47 . \mathrm{IR}(\mathrm{KBr})$ : 1638.08, 1618.05, 1384.50, 1186.27, 834.43, 619.85, 477.54. HRMS (ESI): m/z $[\mathrm{M}+\mathrm{H}]^{+}$calcd for $\mathrm{C}_{17} \mathrm{H}_{12} \mathrm{NCl}_{2}: 300.0344$; found: 300.0341 .<smiles>Brc1ccc(-c2cncc(-c3ccc(Br)cc3)c2)cc1</smiles>

3,5-bis(4-bromophenyl)pyridine (5d):

Pale yellow solid (193 mg, 75\%); mp = 216-218 ${ }^{\circ} \mathrm{C} ;{ }^{1} \mathrm{H}$ NMR $\left(600 \mathrm{MHz}, \mathrm{CDCl}_{3}\right) \delta$ $8.80(\mathrm{~s}, 2 \mathrm{H}), 7.98(\mathrm{~s}, 1 \mathrm{H}), 7.64(\mathrm{~d}, J=7.8 \mathrm{~Hz}, 4 \mathrm{H}), 7.50(\mathrm{~d}, J=7.2 \mathrm{~Hz}, 4 \mathrm{H}) .{ }^{13} \mathrm{C}$ NMR $\left(150 \mathrm{MHz}, \mathrm{CDCl}_{3}\right) \delta 146.73,136.26,135.73,132.61,132.33,128.76,122.87$, IR (KBr): 1635.01, 1488.86, 1382.14, 1076.85, 1006.17, 823.93, 710.41, 523.70. HRMS (ESI): $\mathrm{m} / \mathrm{z}[\mathrm{M}+\mathrm{H}]^{+}$calcd for $\mathrm{C}_{17} \mathrm{H}_{12} \mathrm{Br}_{2} \mathrm{~N}$ : 387.9331 ; found: 387.9334 . 


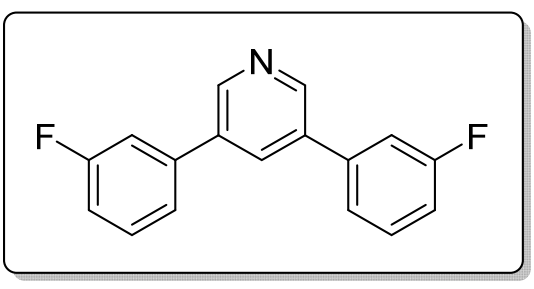

3,5-bis(3-fluorophenyl)pyridine (5e):

Yellow solid (124 mg, 70\%); mp = 131-133 ${ }^{\circ} \mathrm{C} ;{ }^{1} \mathrm{H}$ NMR $\left(600 \mathrm{MHz}, \mathrm{CDCl}_{3}\right) \delta 8.83(\mathrm{~s}$, $2 \mathrm{H}), 8.01(\mathrm{~s}, 1 \mathrm{H}), 7.48(\mathrm{~m}, 2 \mathrm{H}), 7.42(\mathrm{~m}, 2 \mathrm{H}), 7.34(\mathrm{~m}, 2 \mathrm{H}), 7.14(\mathrm{~m}, 2 \mathrm{H}) .{ }^{13} \mathrm{C} \mathrm{NMR}$ $\left(150 \mathrm{MHz}, \mathrm{CDCl}_{3}\right) \delta 164.04,162.40,147.21,139.59(d, J=7.2 \mathrm{~Hz}), 135.58,132.92$, $130.75(d, J=8.4 \mathrm{~Hz}), 122.89,115.26(d, J=21.1 \mathrm{~Hz}), 115.17-114.89(\mathrm{~m}), 114.19$ $(d, J=22.3 \mathrm{~Hz})$, IR (KBr): 1609.28, 1583.89, 1489.47, 1418.45, 1391.58, 1267.31, 1181.80, 1153.60, 1022.37, 974.97, 941.85, 874.69, 795.03, 764.65, 716.43, 692.81, 501.86, 469.97.HRMS (ESI): m/z [M+H]+ calcd for C17H12NF2: 268.0935; found: 268.0932 .

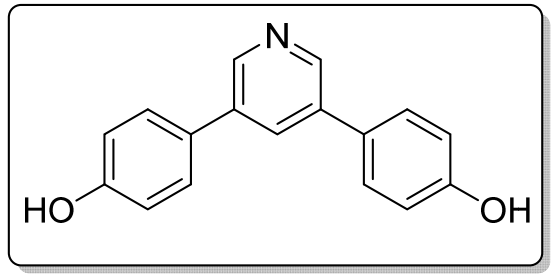

4,4'-(pyridine-3,5-diyl)diphenol (5f):

Brown solid (116 mg, 66\%); mp $=71-74{ }^{\circ} \mathrm{C} ;{ }^{1} \mathrm{H}$ NMR $\left(600 \mathrm{MHz}, \mathrm{DMSO}-d_{6}\right) \delta 9.71$ (s, 2H), $8.72(\mathrm{~m}, 2 \mathrm{H}), 8.10(\mathrm{~m}, 1 \mathrm{H}), 7.65(\mathrm{~d}, J=8.6 \mathrm{~Hz}, 4 \mathrm{H}), 6.90(\mathrm{~d}, J=8.6 \mathrm{~Hz}, 4 \mathrm{H})$. ${ }^{13} \mathrm{C}$ NMR $\left(150 \mathrm{MHz}, \mathrm{DMSO}-d_{6}\right) \delta 157.87,145.07,135.66,130.51,128.37,127.83$, 116.02. IR (KBr): 2925.64, 1611.74, 1515.68, 1415.31, 1271.62, 1173.57, 1024.70, 830.38, 537.93. HRMS (ESI): $\mathrm{m} / \mathrm{z}[\mathrm{M}+\mathrm{H}]^{+}$calcd for $\mathrm{C}_{17} \mathrm{H}_{14} \mathrm{O}_{2} \mathrm{~N}: 264.1020$; found: 264.1019.

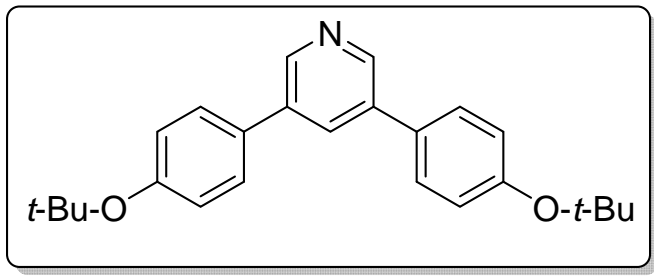

3,5-bis(4-(tert-butoxy)phenyl)pyridine (5g):

Yellow solid (175 mg, 70\%); mp = 134-136 ${ }^{\circ} \mathrm{C} ;{ }^{1} \mathrm{H}$ NMR $\left(600 \mathrm{MHz}, \mathrm{CDCl}_{3}\right) \delta 8.77$ (s, 2H), $8.02(\mathrm{~s}, 1 \mathrm{H}), 7.55(\mathrm{~d}, J=8.4 \mathrm{~Hz}, 4 \mathrm{H}), 7.12$ (d, $J=7.8 \mathrm{~Hz}, 4 \mathrm{H}), 1.40(\mathrm{~s}, 18 \mathrm{H})$. ${ }^{13} \mathrm{C} \mathrm{NMR}\left(150 \mathrm{MHz}, \mathrm{CDCl}_{3}\right) \delta 155.80,146.08,136.22,132.48,127.71,124.56,78.96$, 28.87. IR (KBr): 2975.24, 1604.68, 1506.83, 1387.90, 1365.64, 1302.42, 1236.18, 1168.11, 1044.56, 1014.99, 898.67, 854.08, 687.89, 619.07, 569.78. HRMS (ESI): $\mathrm{m} / \mathrm{z}[\mathrm{M}+\mathrm{H}]^{+}$calcd for $\mathrm{C}_{25} \mathrm{H}_{30} \mathrm{O}_{2} \mathrm{~N}: 376.2274$; found: 376.2271 . 


\section{Crystalline solidlographic data and molecular structure of compound $3 \mathrm{~h}$ (4-ethyl-2,6-di(naphthalen-2-yl)pyridine)}

$\mathrm{X}$-ray structure determination was obtained via slow evaporation of compound $3 \mathrm{~h}$ in $\mathrm{CHCl}_{3} / \mathrm{MeOH}$

$(5: 1)$ at room temperature.
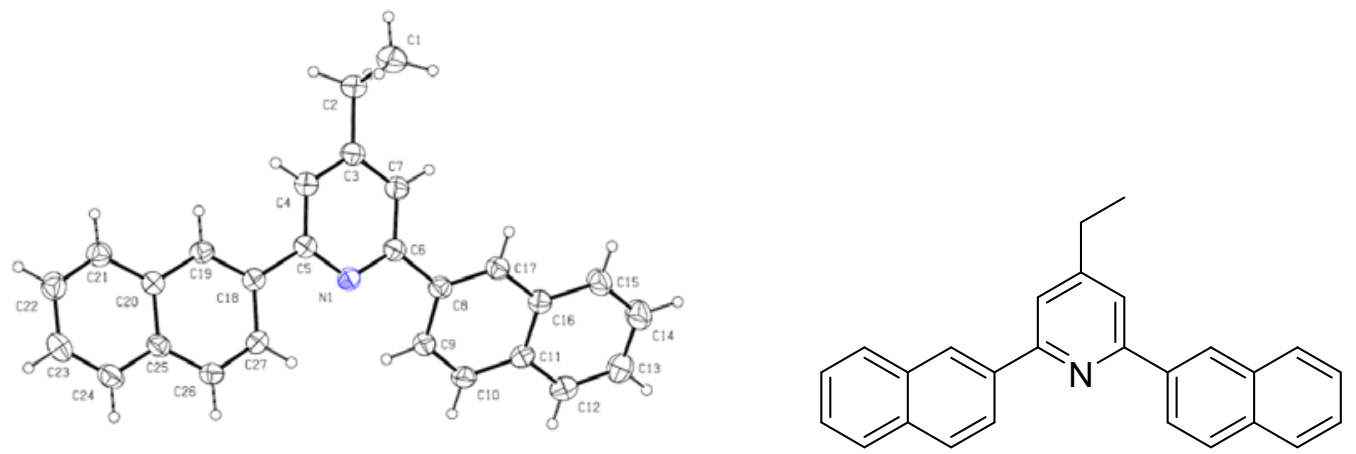

Figure S3 X-ray crystalline solid structure of $\mathbf{3 h}$; ellipsoids depicted at the 50\% probability level.

Table S1. Crystalline solid data and structure refinement for compound 3h (CCDC: 1420909)

\begin{tabular}{|c|c|c|c|c|}
\hline $\begin{array}{l}\text { Empirical } \\
\text { formula }\end{array}$ & \multicolumn{2}{|c|}{$\mathrm{C}_{27} \mathrm{H}_{21} \mathrm{~N}$} & Absorption coefficient & $0.072 \mathrm{~mm}^{-1}$ \\
\hline Formula weight & \multicolumn{2}{|c|}{359.45} & $\mathrm{~F}(000)$ & 760 \\
\hline Temperature & \multicolumn{2}{|c|}{$296(2)$} & Crystalline solid size & $0.12 \times 0.10 \times 0.10 \mathrm{~mm}^{3}$ \\
\hline Wavelength & \multicolumn{2}{|c|}{0.71073} & Reflections collected & 14071 \\
\hline $\begin{array}{l}\text { Crystalline solid } \\
\text { system }\end{array}$ & \multicolumn{2}{|c|}{ Monoclinic } & $\begin{array}{l}\text { Independent } \\
\text { reflections }\end{array}$ & $4154[\mathrm{R}($ int $)=0.0491]$ \\
\hline Space group & \multicolumn{2}{|c|}{$\mathrm{P} 21 / \mathrm{c}$} & $\begin{array}{l}\text { Max. and min. } \\
\text { transmission }\end{array}$ & 0.9928 and 0.9914 \\
\hline \multirow{3}{*}{$\begin{array}{c}\text { Unit cell } \\
\text { dimensions }\end{array}$} & $\begin{array}{c}\mathrm{a}= \\
9.829(2) \\
\AA\end{array}$ & $\begin{array}{c}\alpha= \\
90.00(4)^{\circ}\end{array}$ & Refinement method & $\begin{array}{l}\text { Full-matrix least-squares } \\
\text { on } \mathrm{F}^{2}\end{array}$ \\
\hline & $\begin{array}{c}\mathrm{b}= \\
17.878(4) \\
\AA\end{array}$ & $\begin{array}{c}\beta= \\
102.800( \\
4)^{\circ}\end{array}$ & Goodness-of-fit on $\mathrm{F}^{2}$ & 1.039 \\
\hline & $\begin{array}{c}c= \\
11.147(2) \\
\AA\end{array}$ & $\begin{array}{c}\gamma= \\
90.00(4)^{\circ}\end{array}$ & $\begin{array}{l}\text { Final } R \text { indices } \\
{[\mathrm{I}>2 \operatorname{sigma}(\mathrm{I})]}\end{array}$ & $\begin{array}{c}\mathrm{R} 1=0.0498, \mathrm{wR} 2= \\
0.1671\end{array}$ \\
\hline Volume & \multicolumn{2}{|c|}{$1910.1(7) \AA^{3}$} & $\mathrm{R}$ indices (all data) & $\begin{aligned} \mathrm{R} 1= & 0.1130, \mathrm{wR} 2= \\
& 0.1253\end{aligned}$ \\
\hline $\mathrm{Z}$ & \multicolumn{2}{|c|}{4} & $\begin{array}{c}\text { Largest diff. peak and } \\
\text { hole }\end{array}$ & 0.151 and $-0.160 \mathrm{e}^{-3}$ \\
\hline $\begin{array}{c}\text { Density } \\
\text { (calculated) }\end{array}$ & \multicolumn{2}{|c|}{$1.250 \mathrm{Mg} / \mathrm{m}^{3}$} & CCDC number & 1420909 \\
\hline
\end{tabular}


8. Copies of ${ }^{1} \mathrm{H}$ NMR,${ }^{13} \mathrm{C}$ NMR and HRMS/MS Spectra
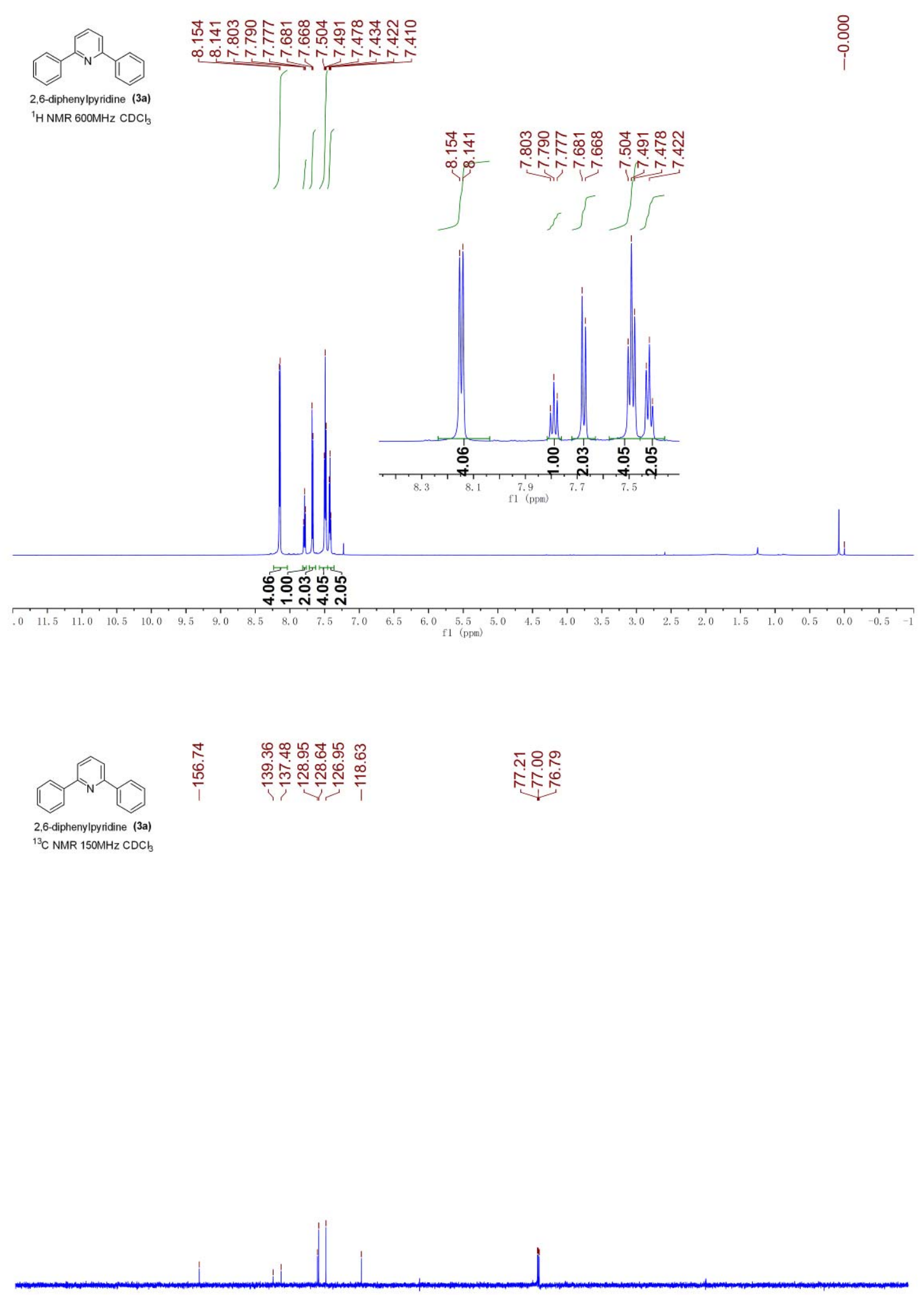

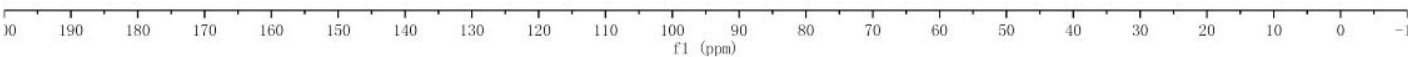



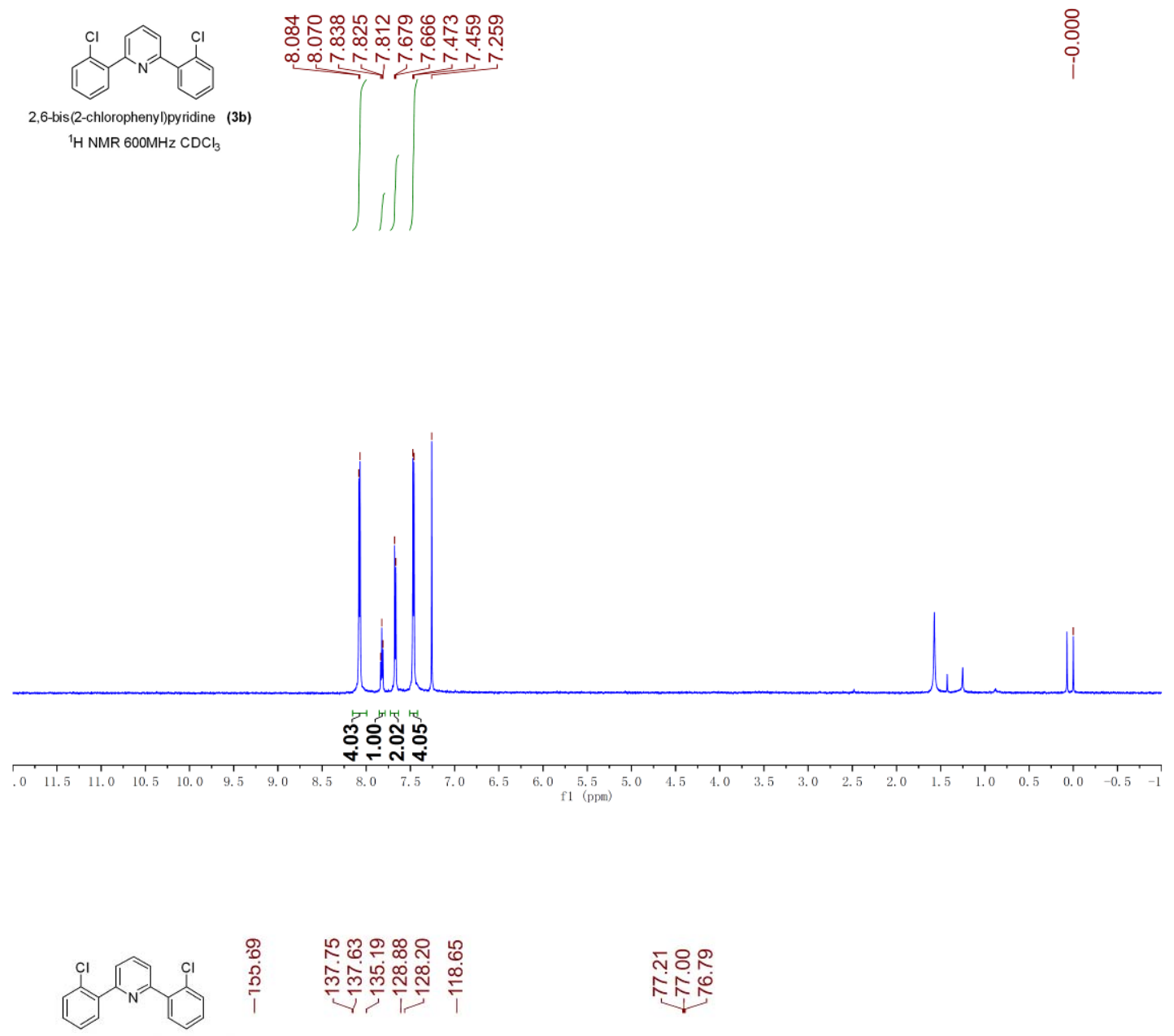

2,6-bis(2-chlorophenyl)pyridine (3b)

${ }^{13} \mathrm{C}$ NMR $150 \mathrm{MHz} \mathrm{CDCl}$

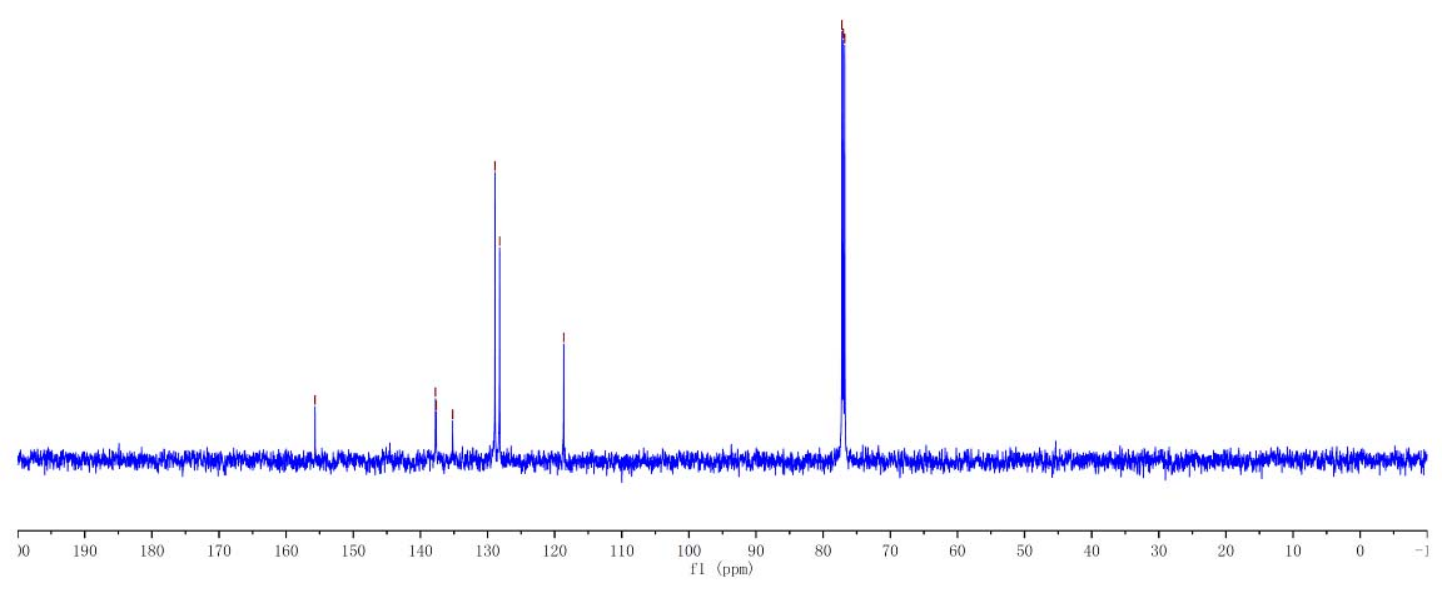




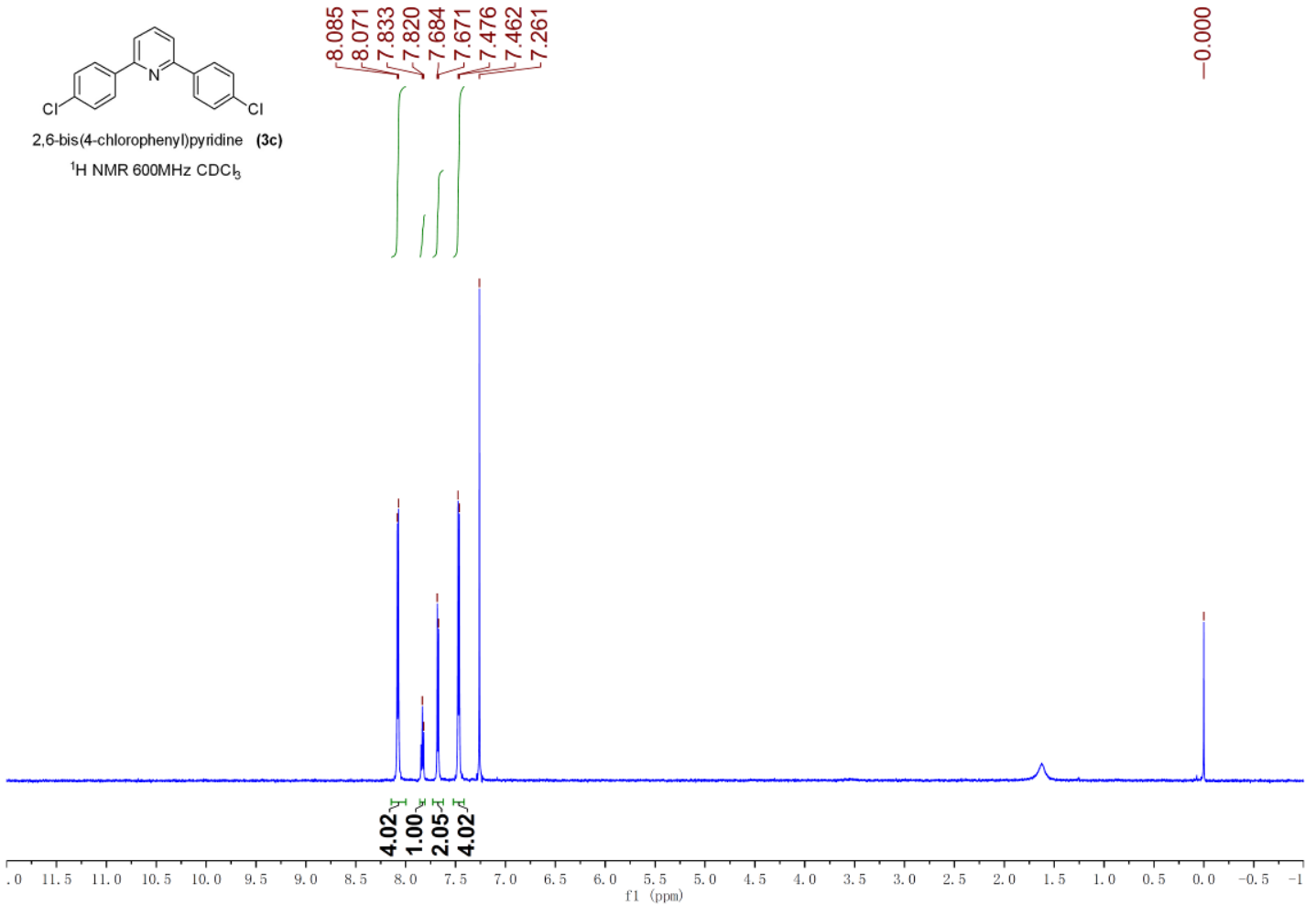

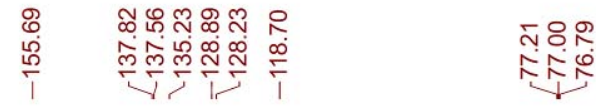

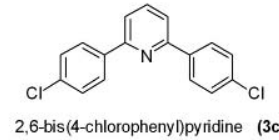

${ }^{13} \mathrm{CNMR} 150 \mathrm{MHz} \mathrm{CDCl}$

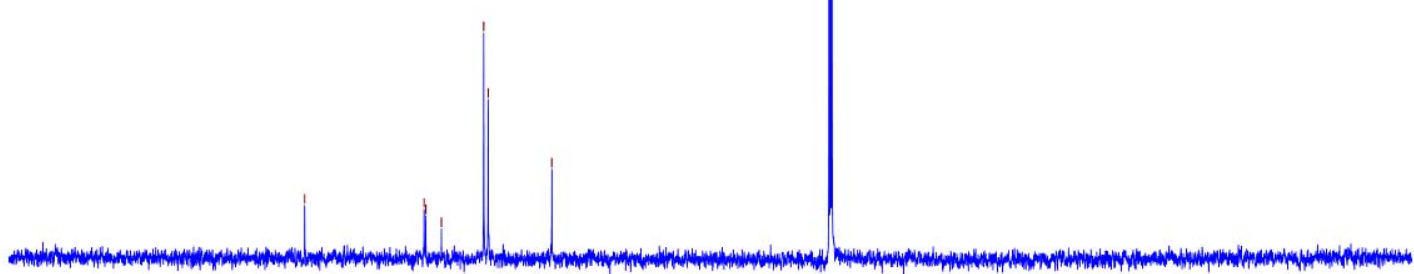

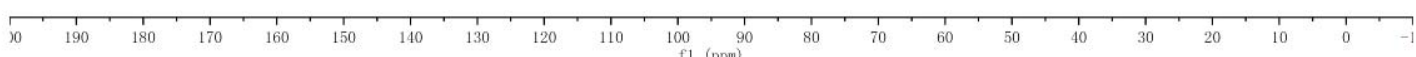



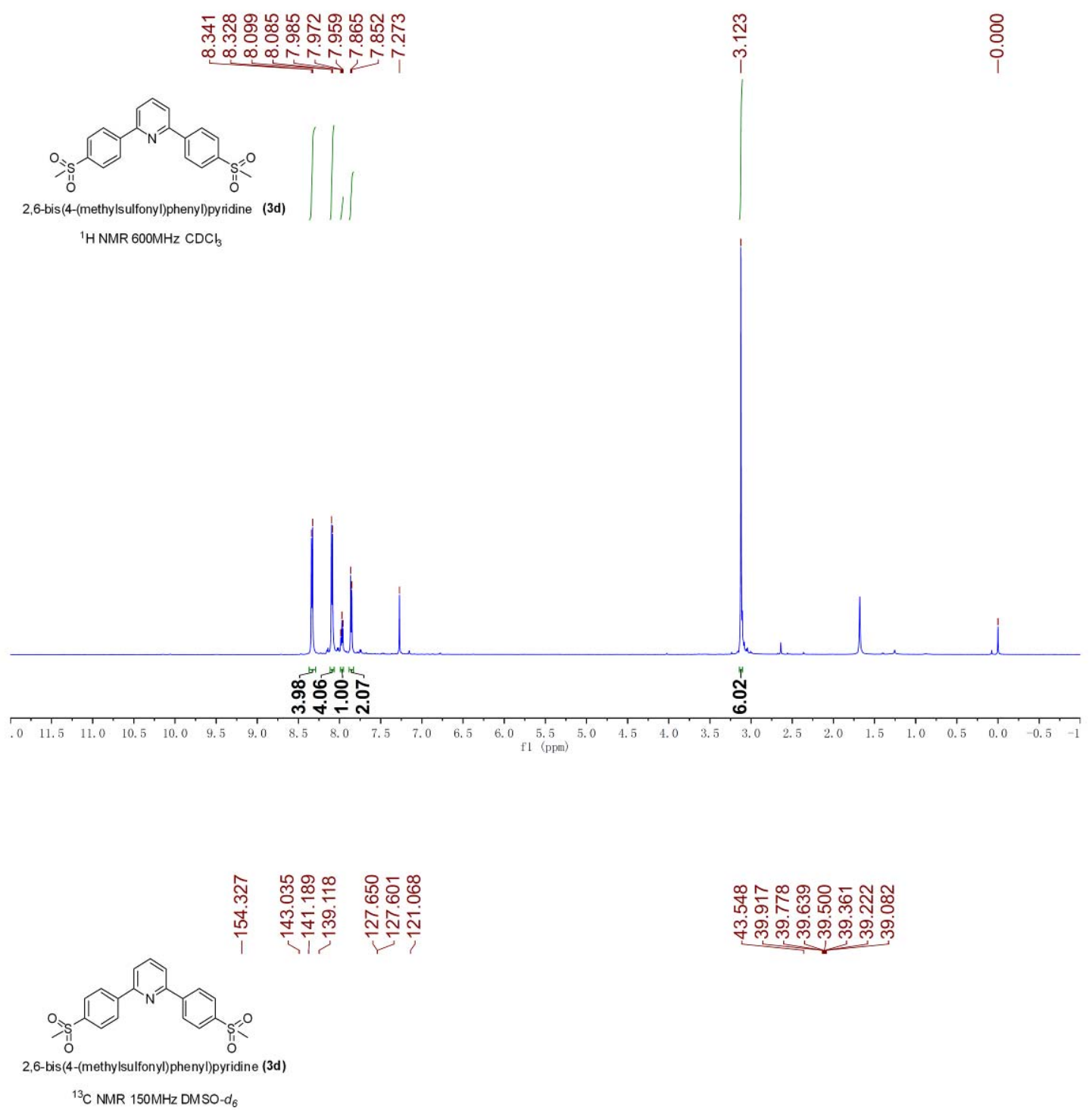

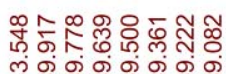

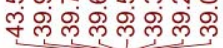

${ }^{13} \mathrm{C}$ NMR $150 \mathrm{MHZ}$ DMSO-d

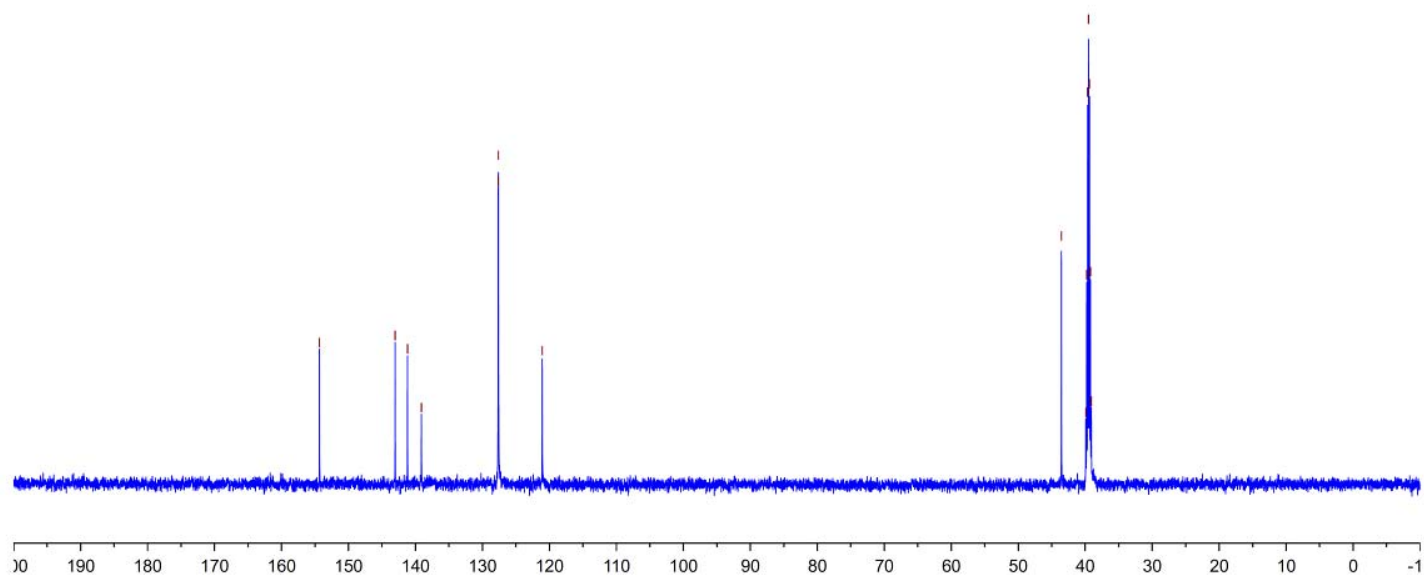



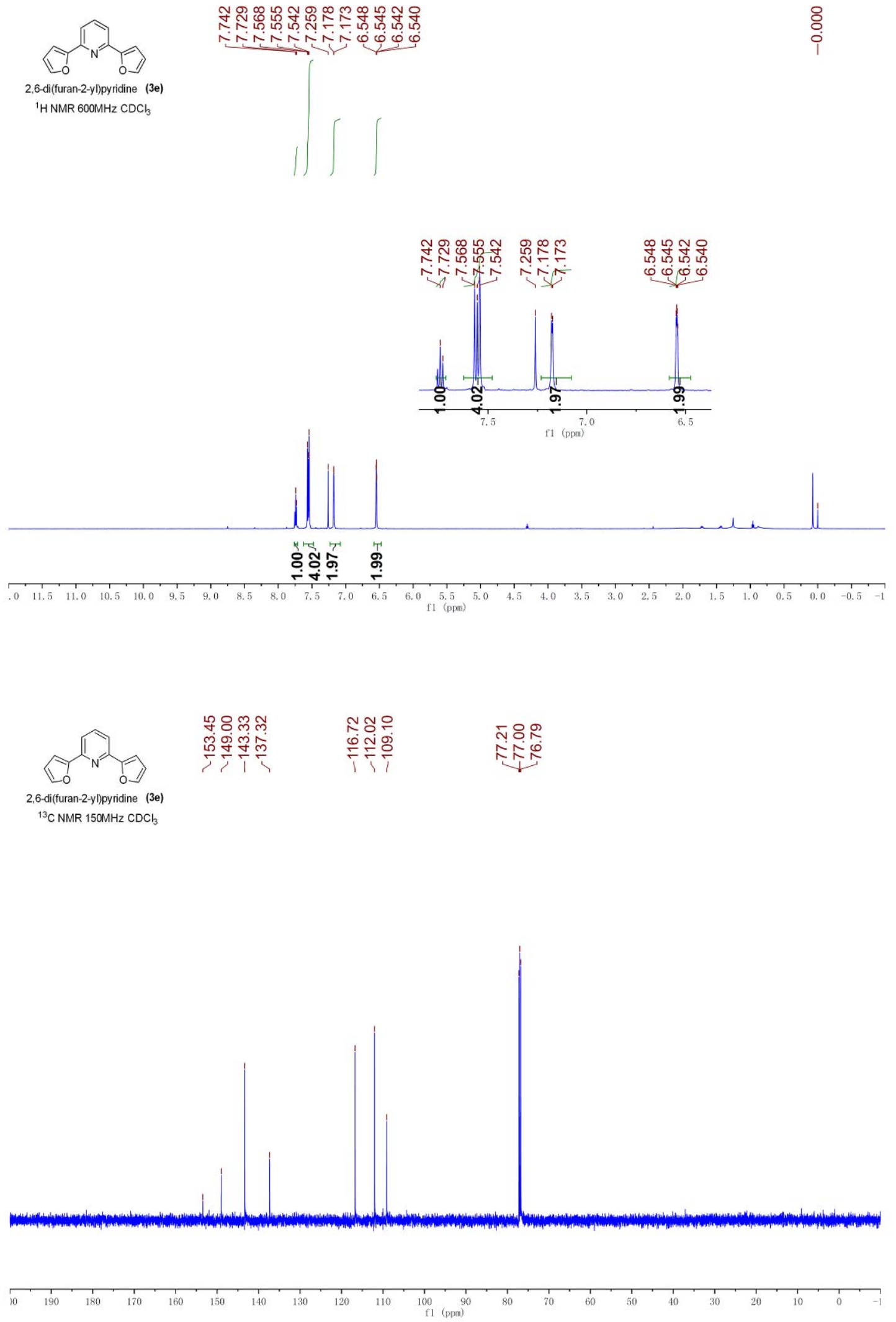

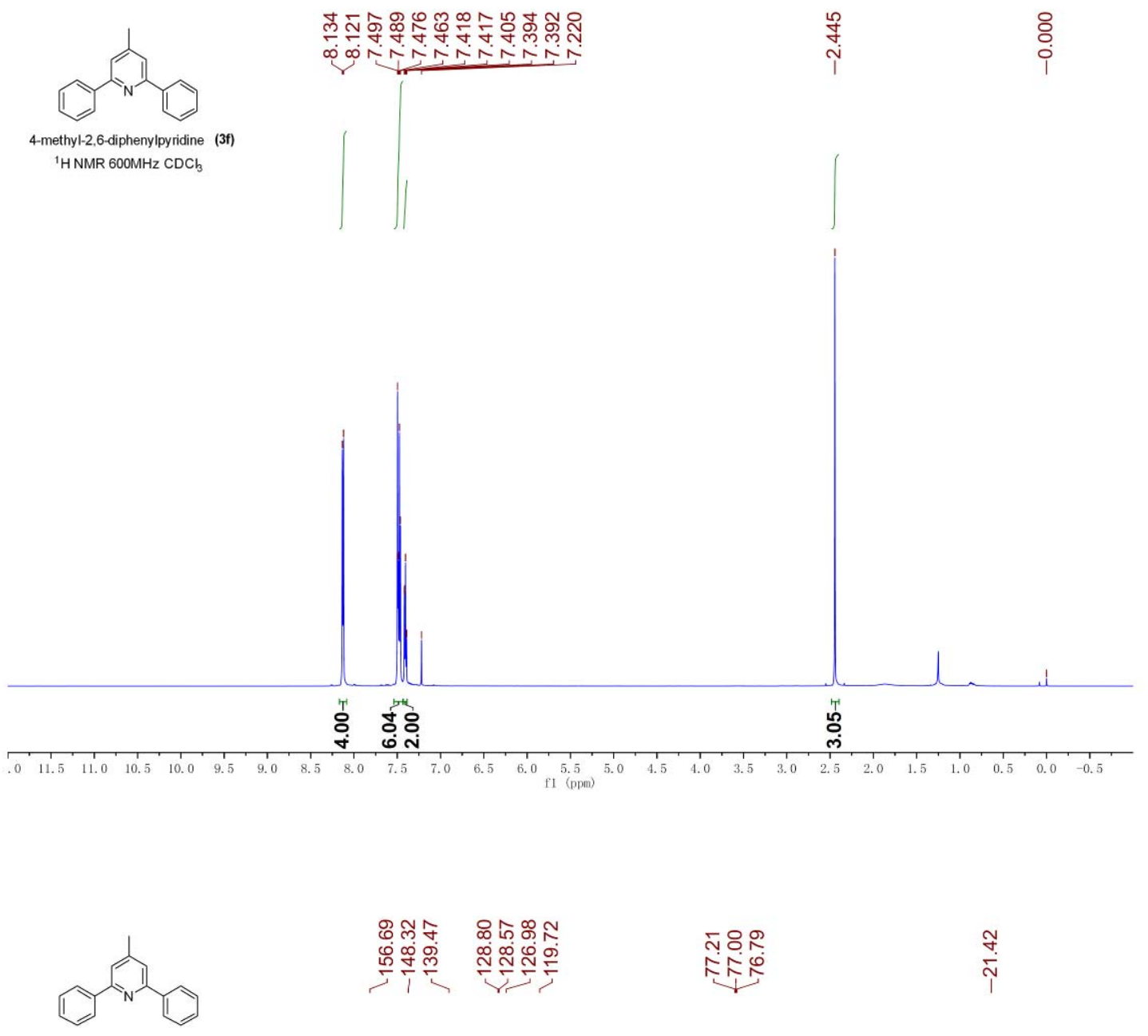

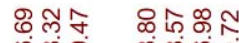

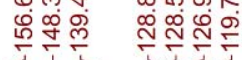

ำดㅇำ

슈요

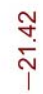

4-methyl-2,6-diphenylpyridine (3f)

${ }^{13} \mathrm{C} \mathrm{NMR} 150 \mathrm{MHz} \mathrm{CDCl}$

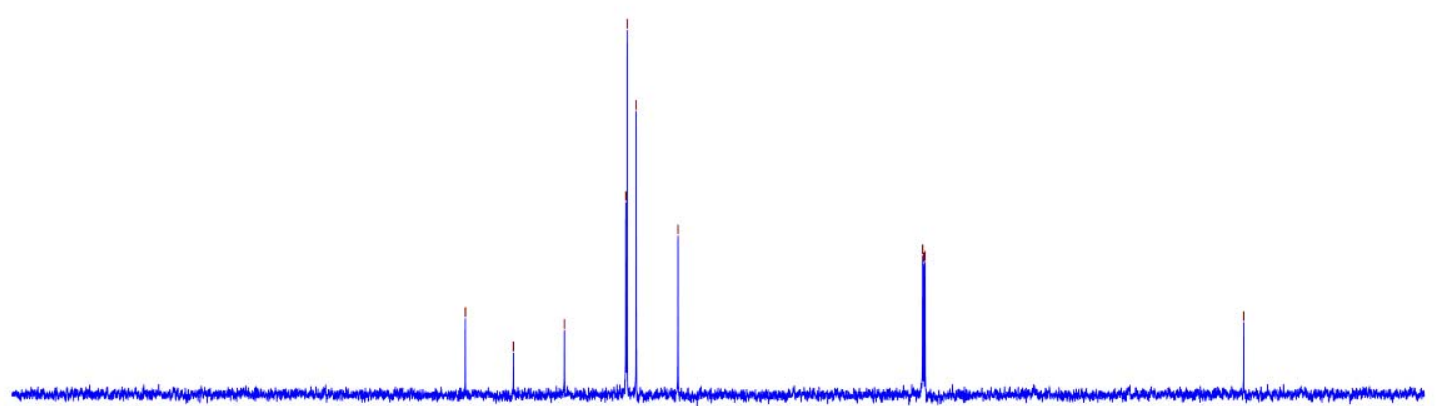

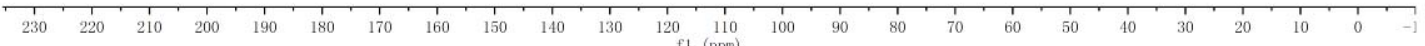




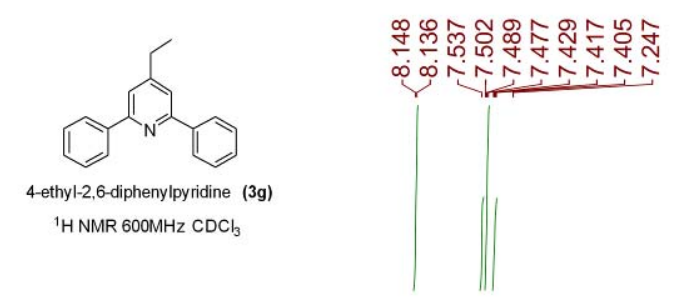

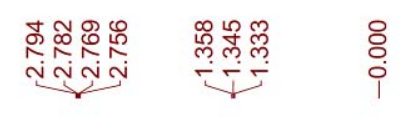

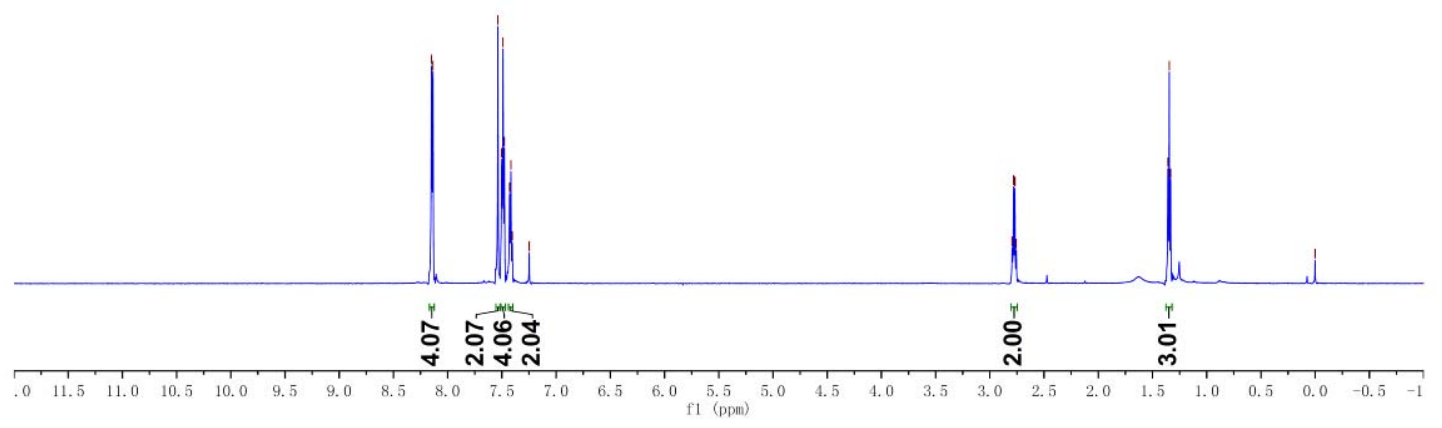

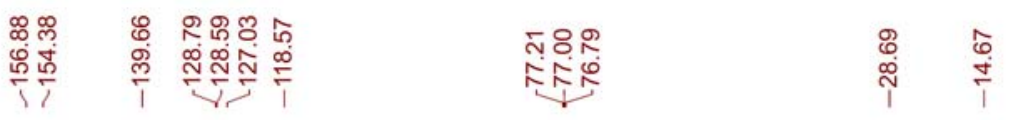

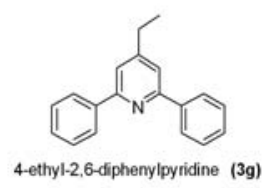

${ }^{13} \mathrm{CNMR} 150 \mathrm{MHz} \mathrm{CDCb}$

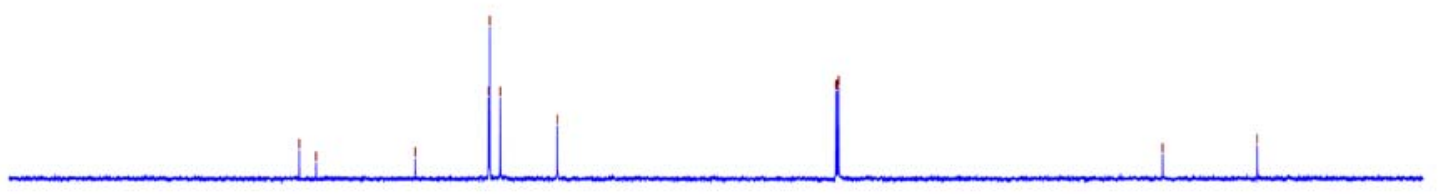

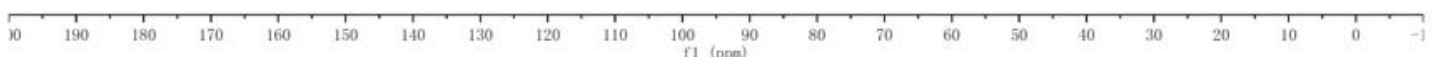




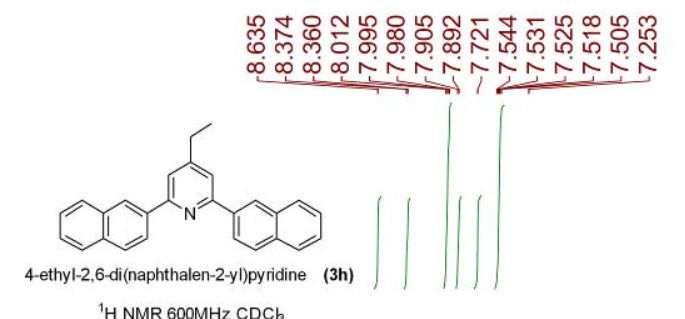

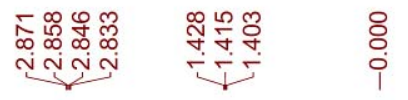
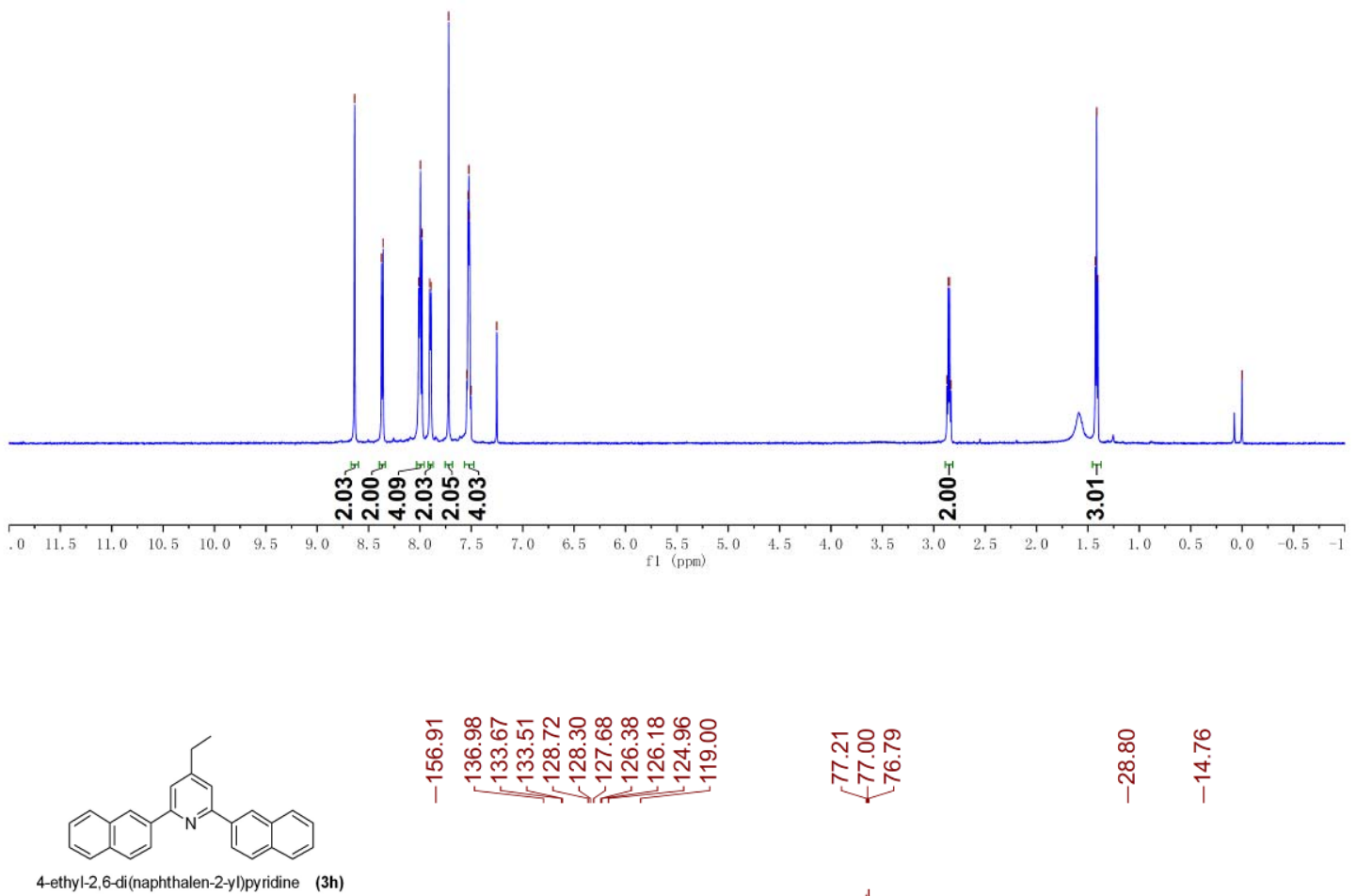

ธ ๘

फ

กุำ

손

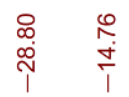

4-ethyl-2,6-di(naphthalen-2-yl)pyridine (3h)

${ }^{13} \mathrm{C} \mathrm{NMR}_{150 \mathrm{MHz}} \mathrm{CDCl}_{3}$

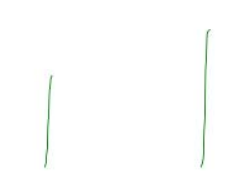



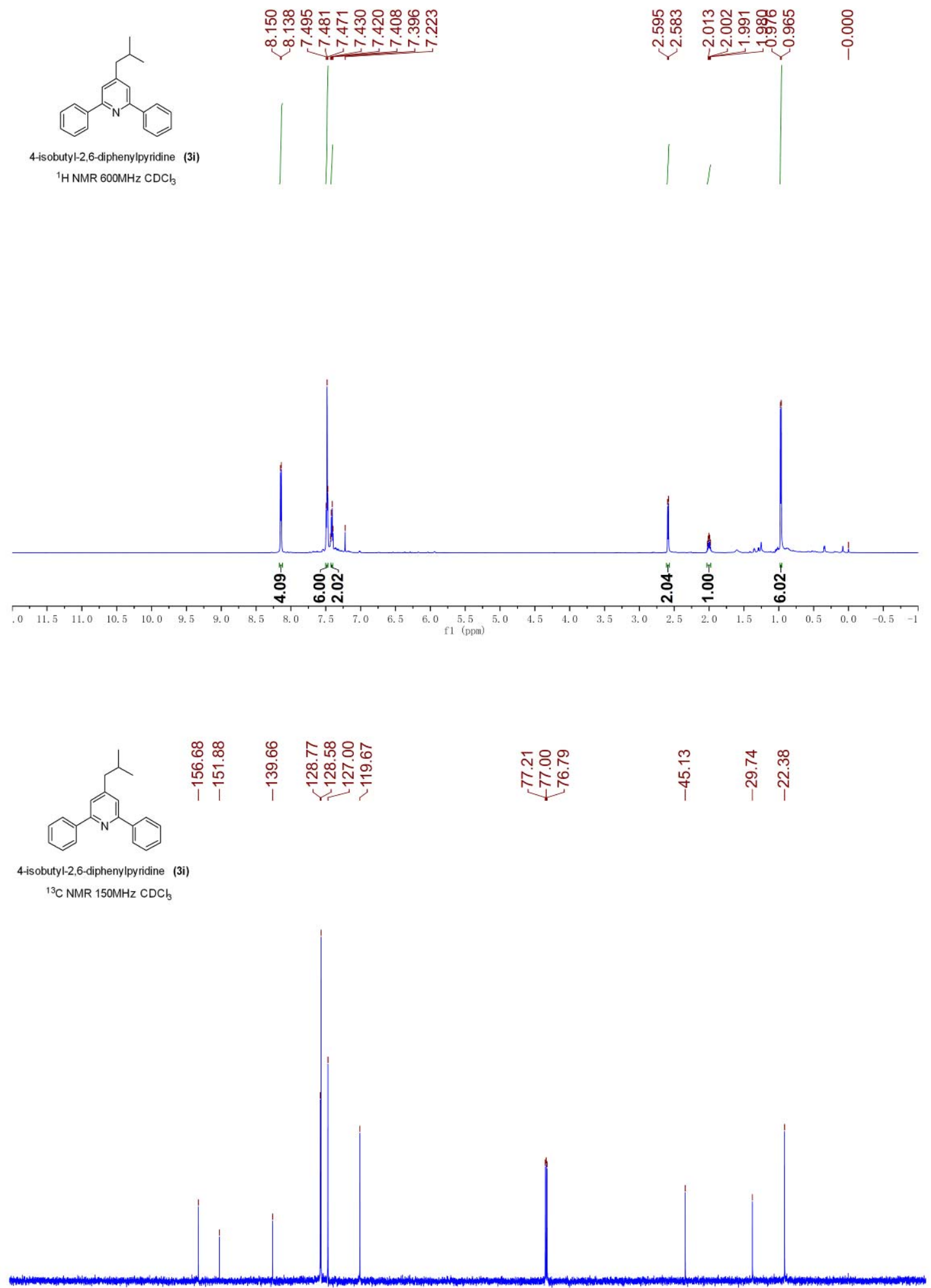

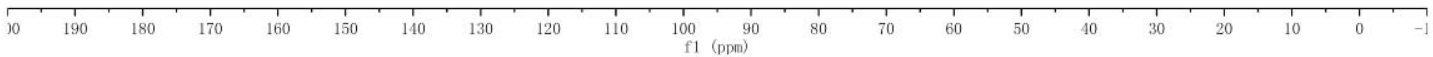



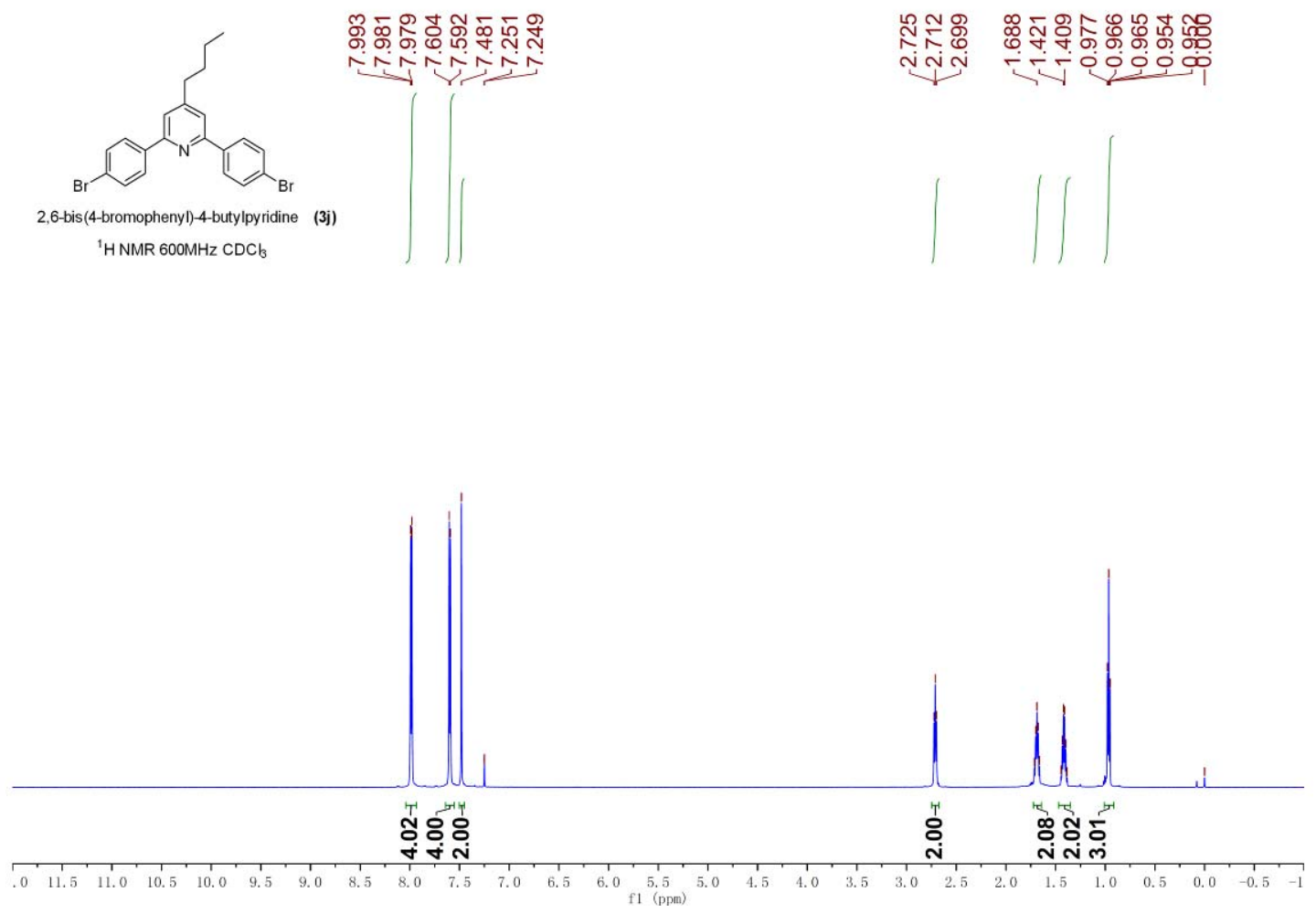

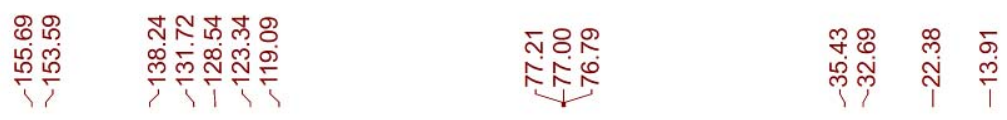

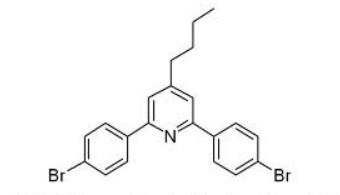

2,6-bis(4-bromopheny) -4-butylpyridine (3i)

${ }^{13} \mathrm{C}$ NMR $150 \mathrm{MHZ} \mathrm{CDCl}$

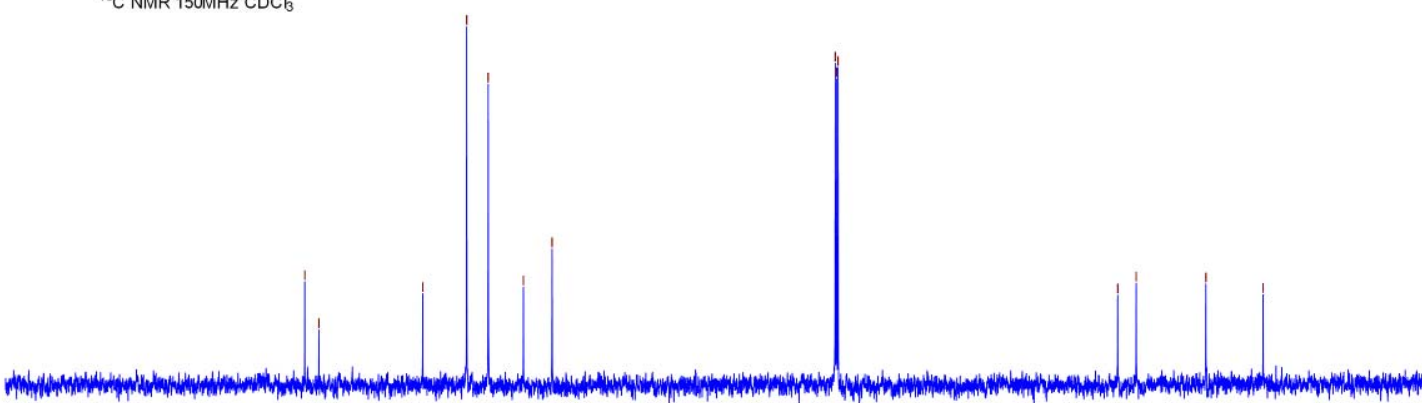

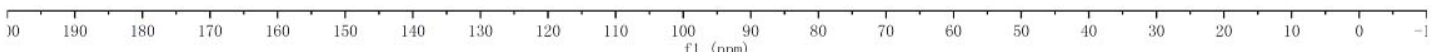



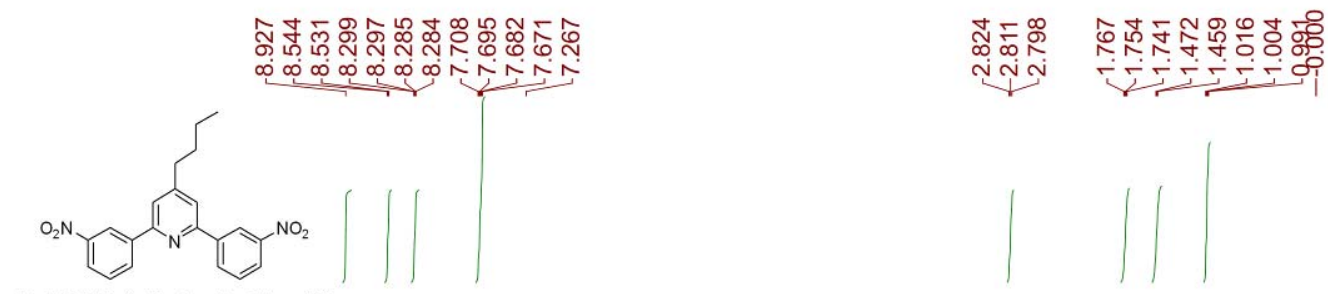

4-butyl-2,6-bis(3-nitrophenyl)pyridine (3k)

${ }^{1} \mathrm{H} \mathrm{NMR} 600 \mathrm{MHz} \mathrm{CDCl}$

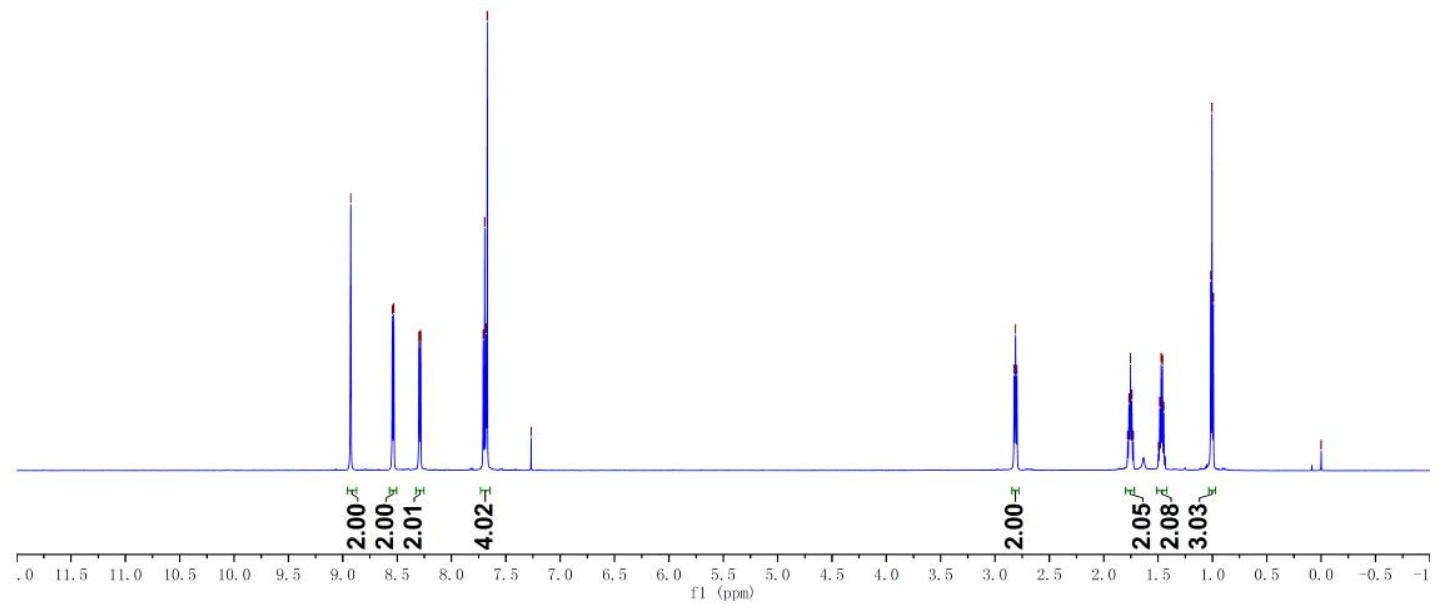

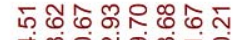

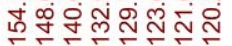

다유옹

年负

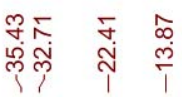

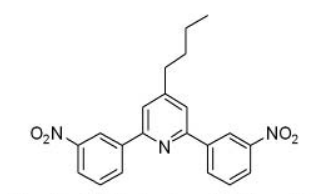

4-butyl-2,6-bis(3-nitrophenyl)pyridine (3k)

${ }^{13} \mathrm{C}$ NMR $150 \mathrm{MHz} \mathrm{CDCl}$

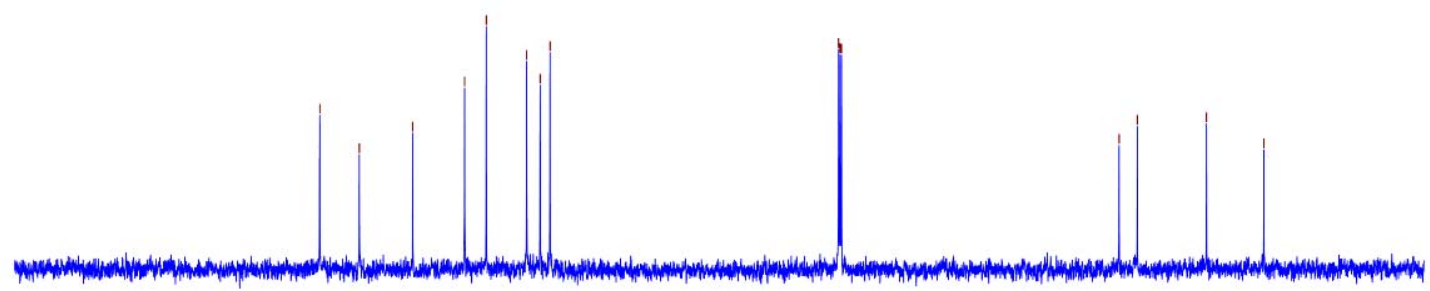

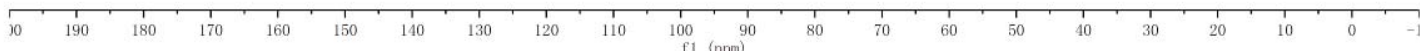



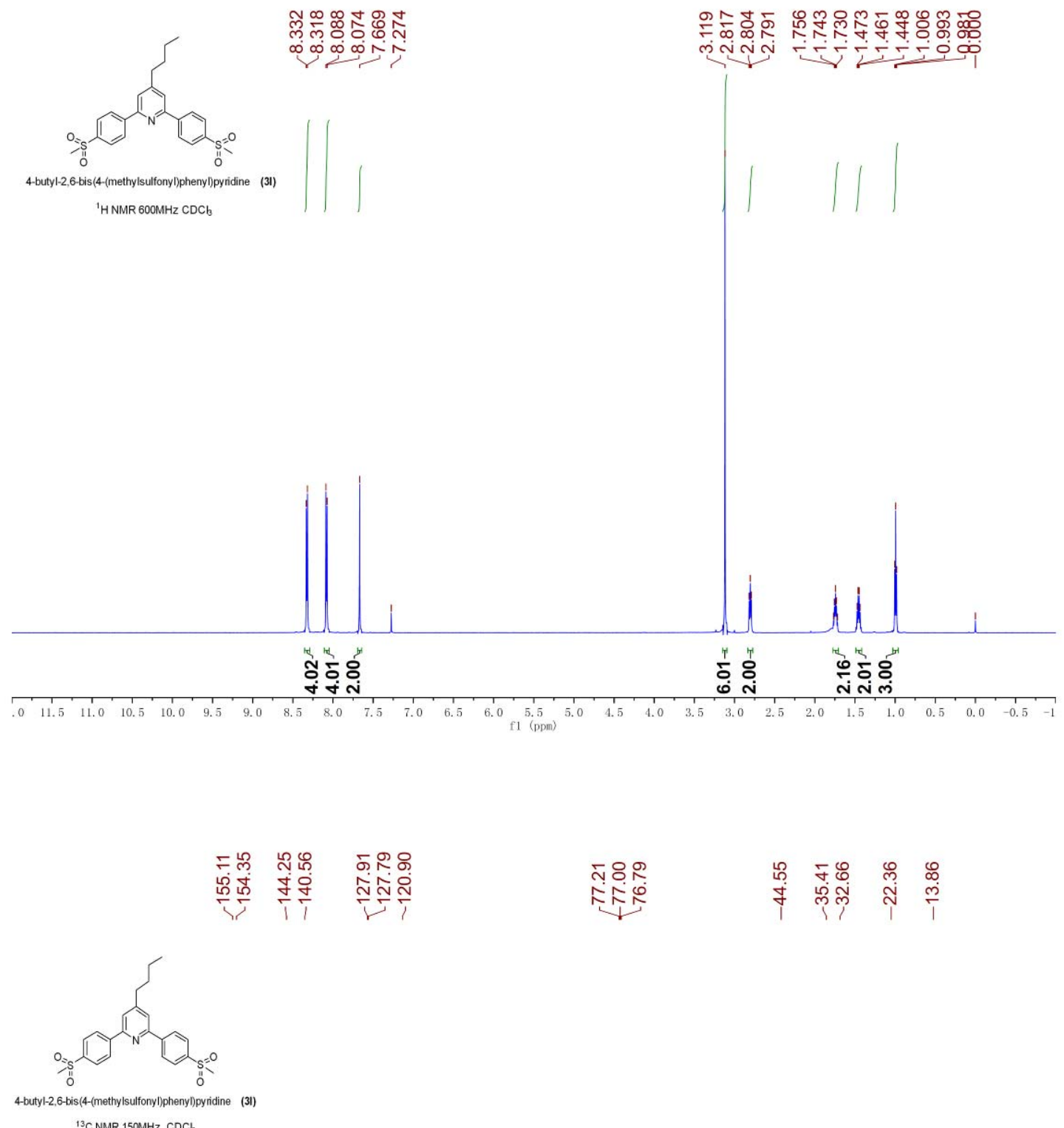

${ }^{13} \mathrm{CNMR} 15 \mathrm{MHz} \mathrm{CDCl}_{3}$

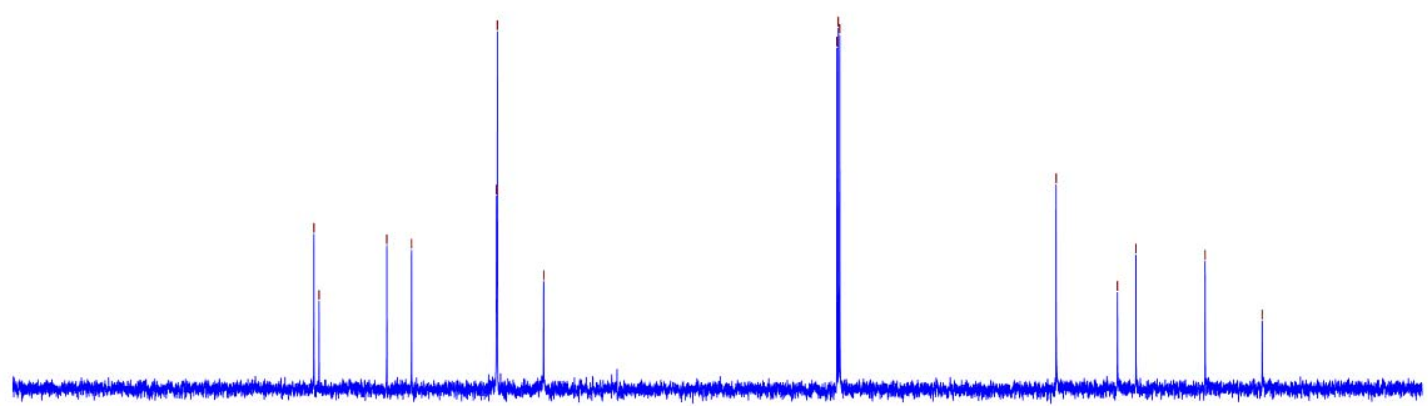




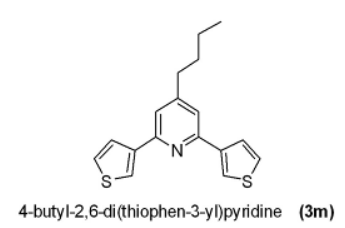

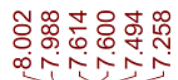

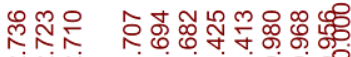

तin

phen-3-yl)pyridine $(3 \mathrm{~m})$

$\mathrm{H}$ NMR $600 \mathrm{MHz} \mathrm{CDCl}$
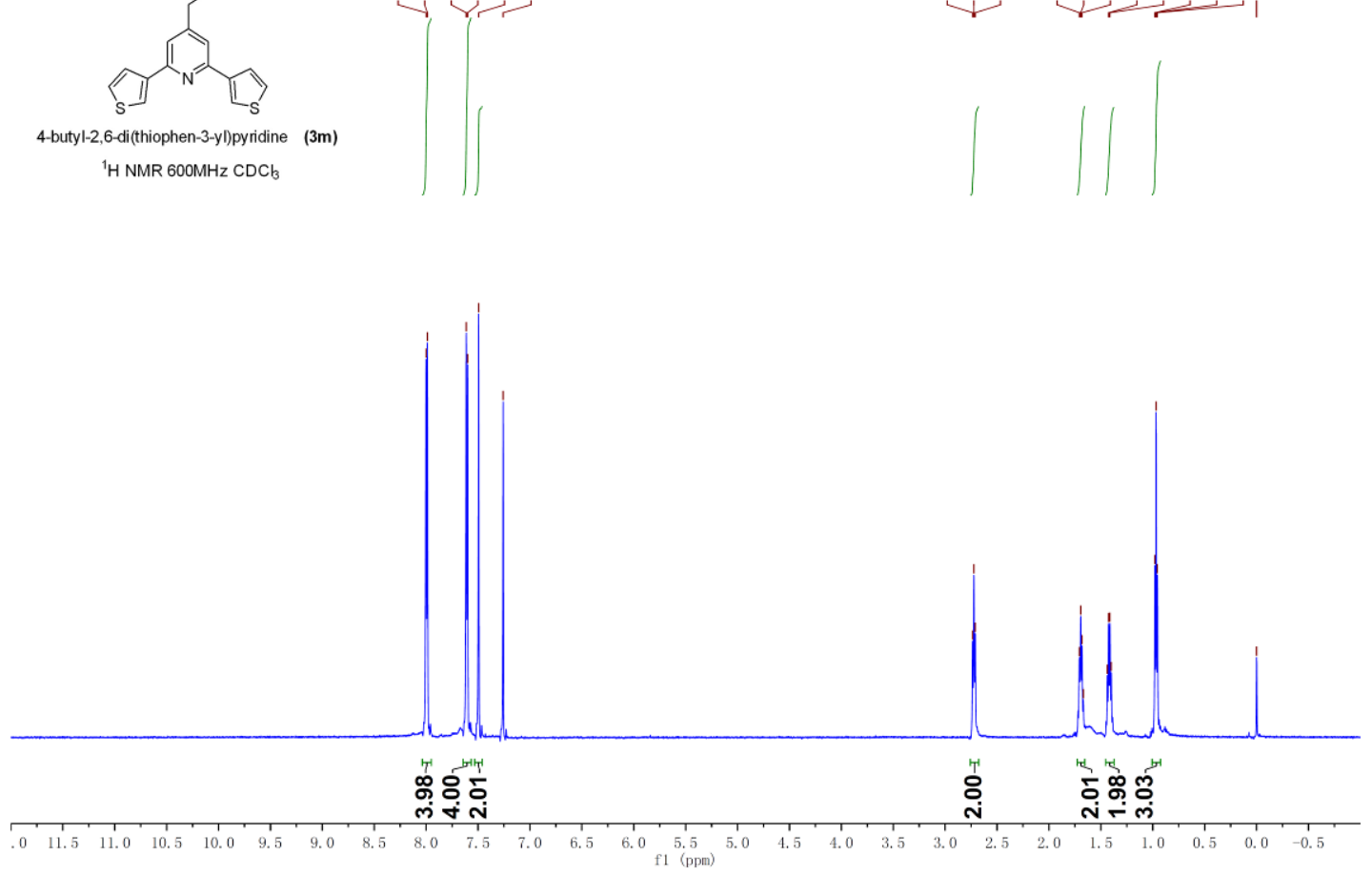

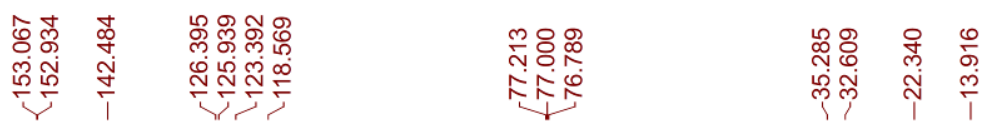

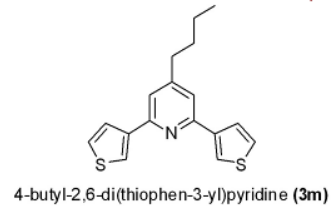

4-butyl-2,6-di(thiophen-3-yl)pyridine (3m)

${ }^{13} \mathrm{C} \mathrm{NMR} 150 \mathrm{MHz} \mathrm{CDCl}_{3}$

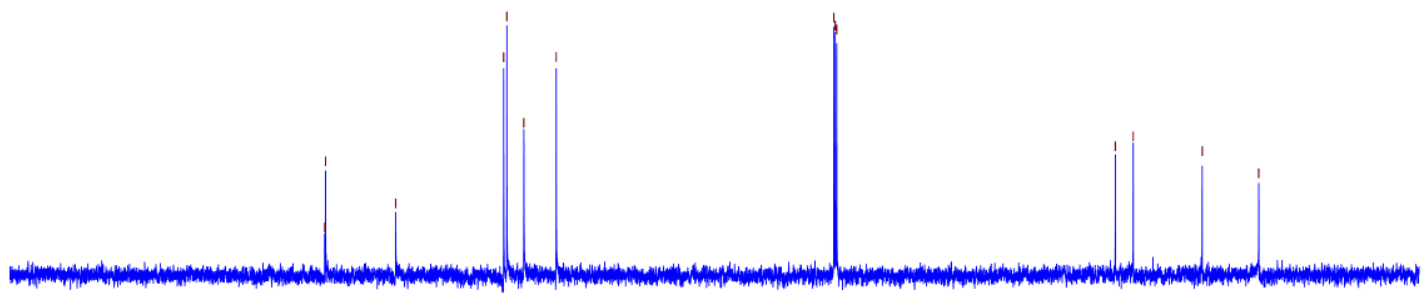

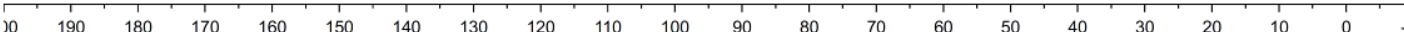




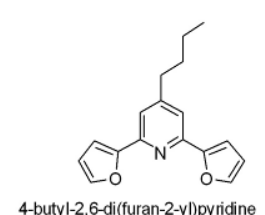

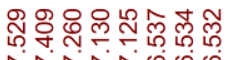

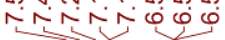

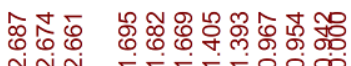

๙

4-butyl-2,6-di(furan-2-yl)pyridine (3n)

'H NMR 600MHz CDCb
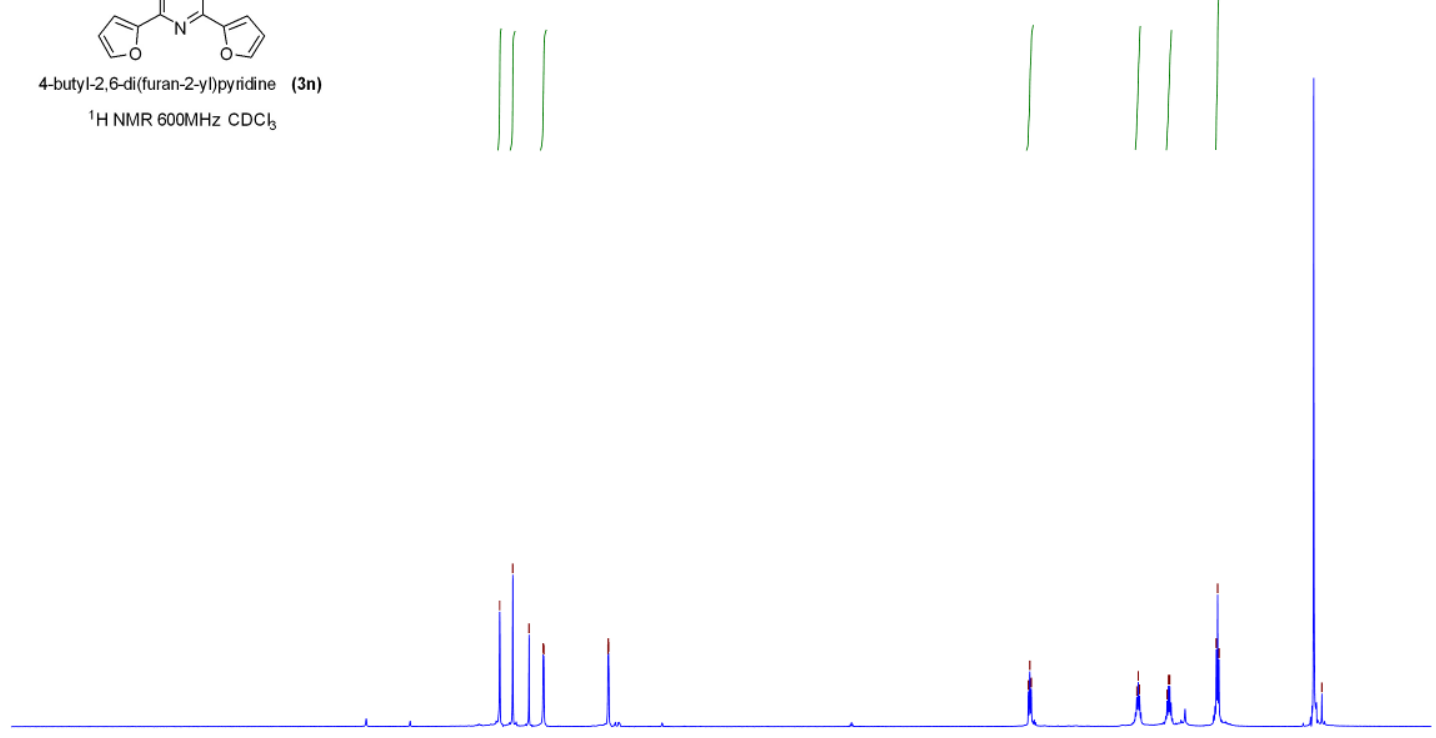

รัต"

ถ

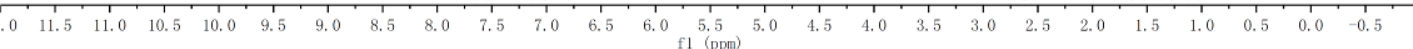
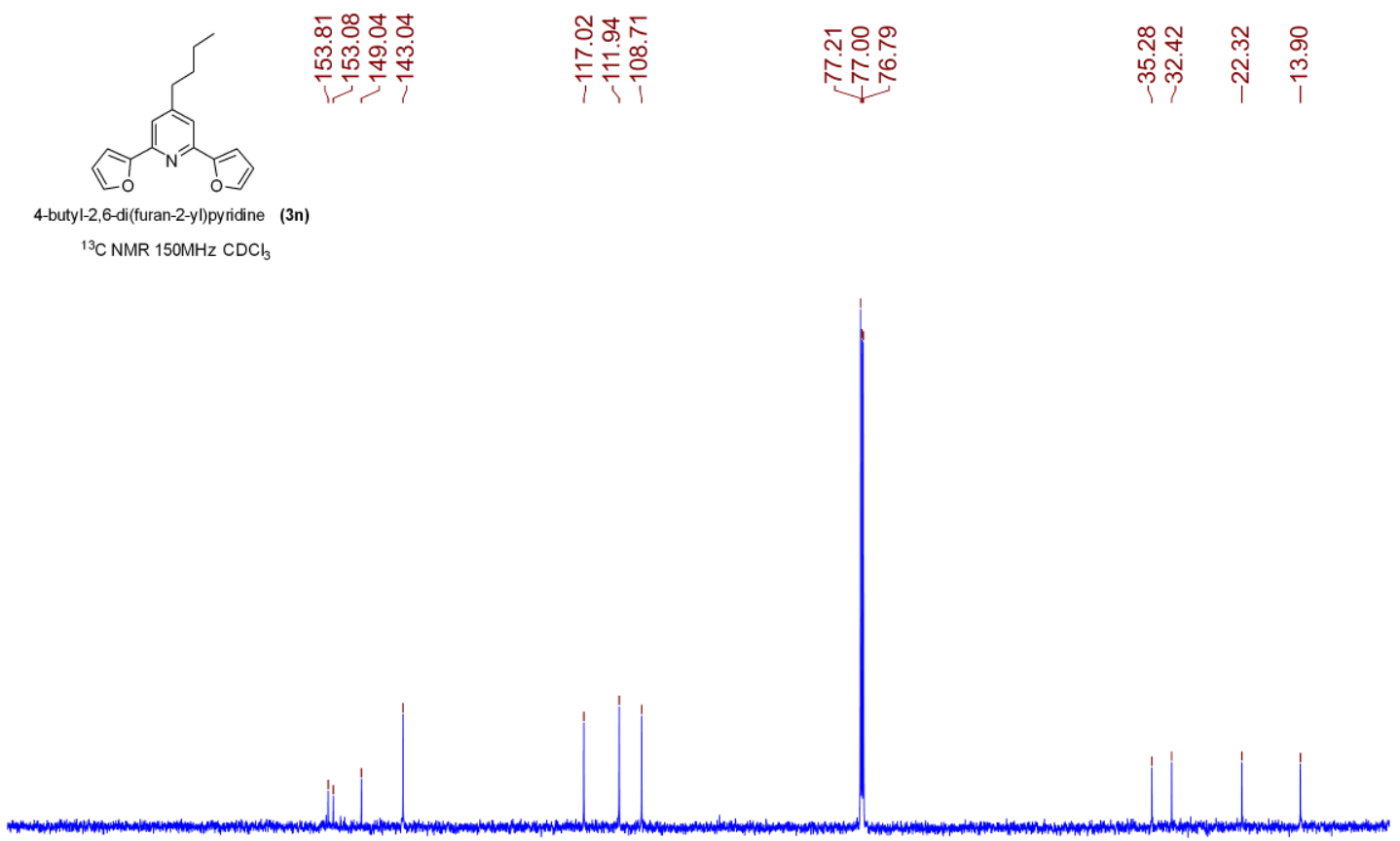

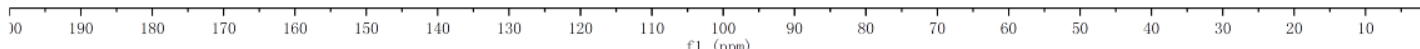




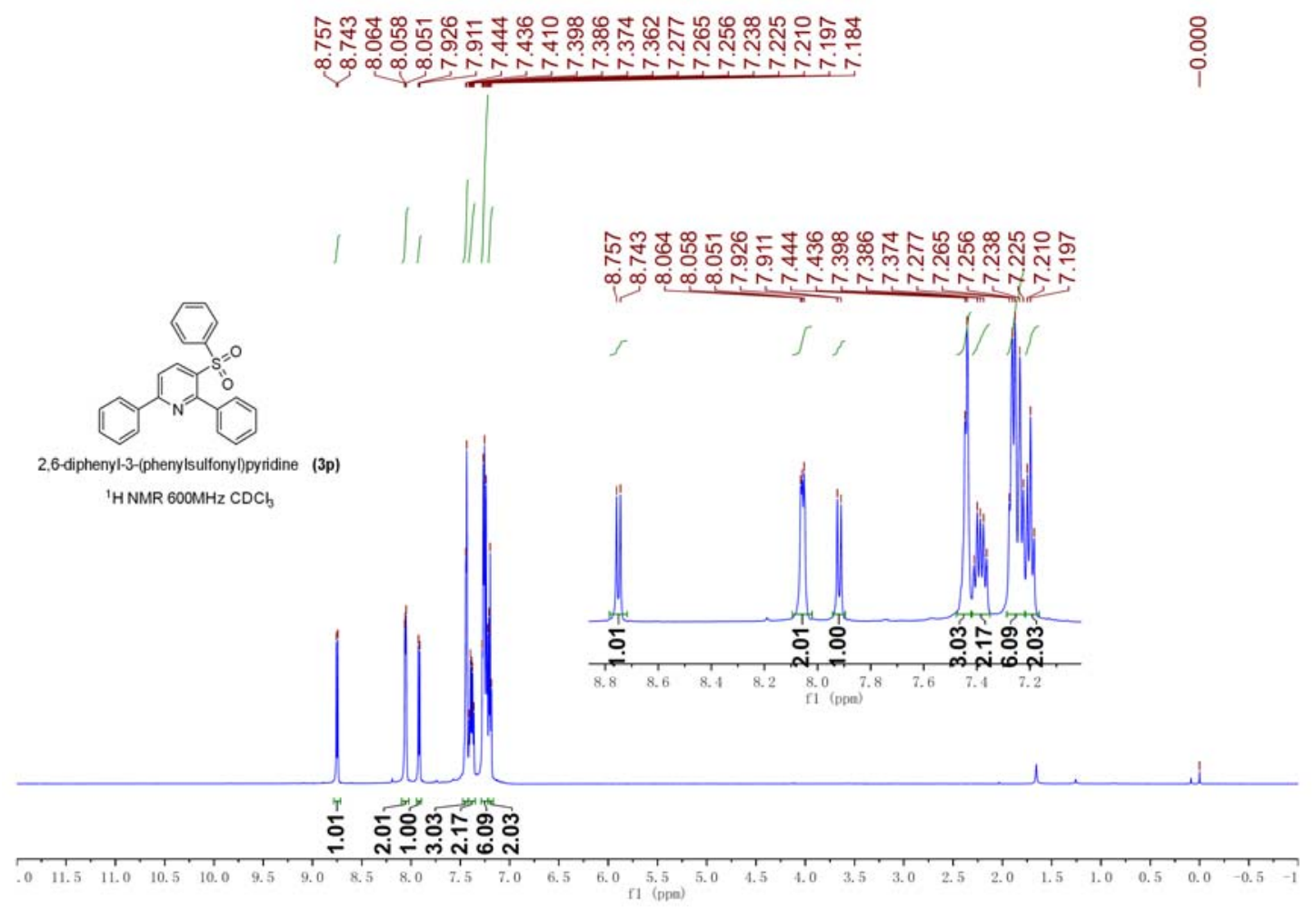

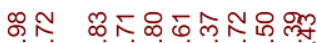

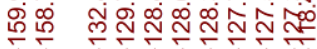

స̦ำ

篎

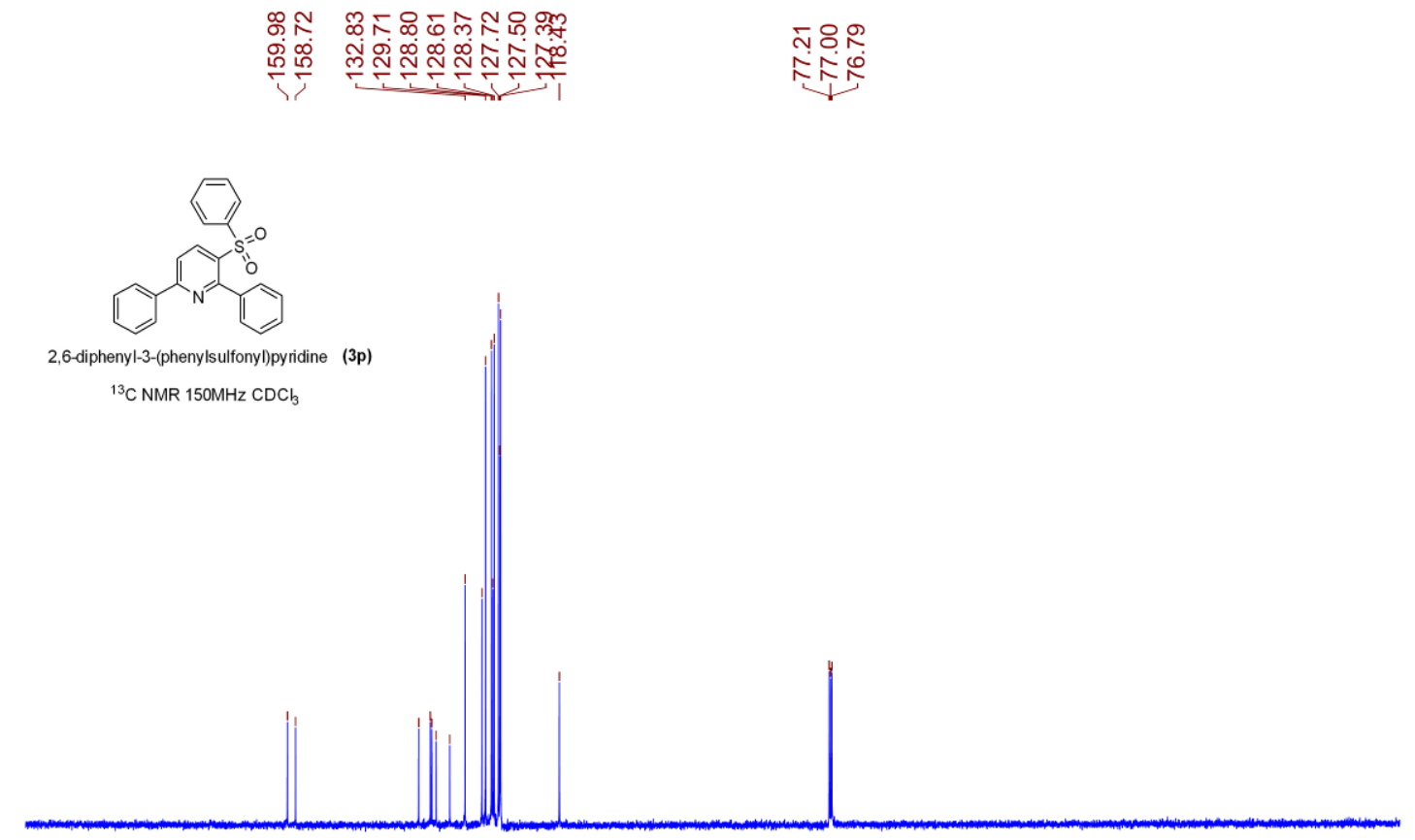

${ }^{13} \mathrm{C} \mathrm{NMR} 150 \mathrm{MHz} \mathrm{CDCl}$

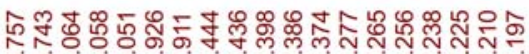

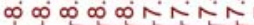

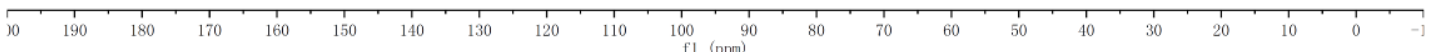




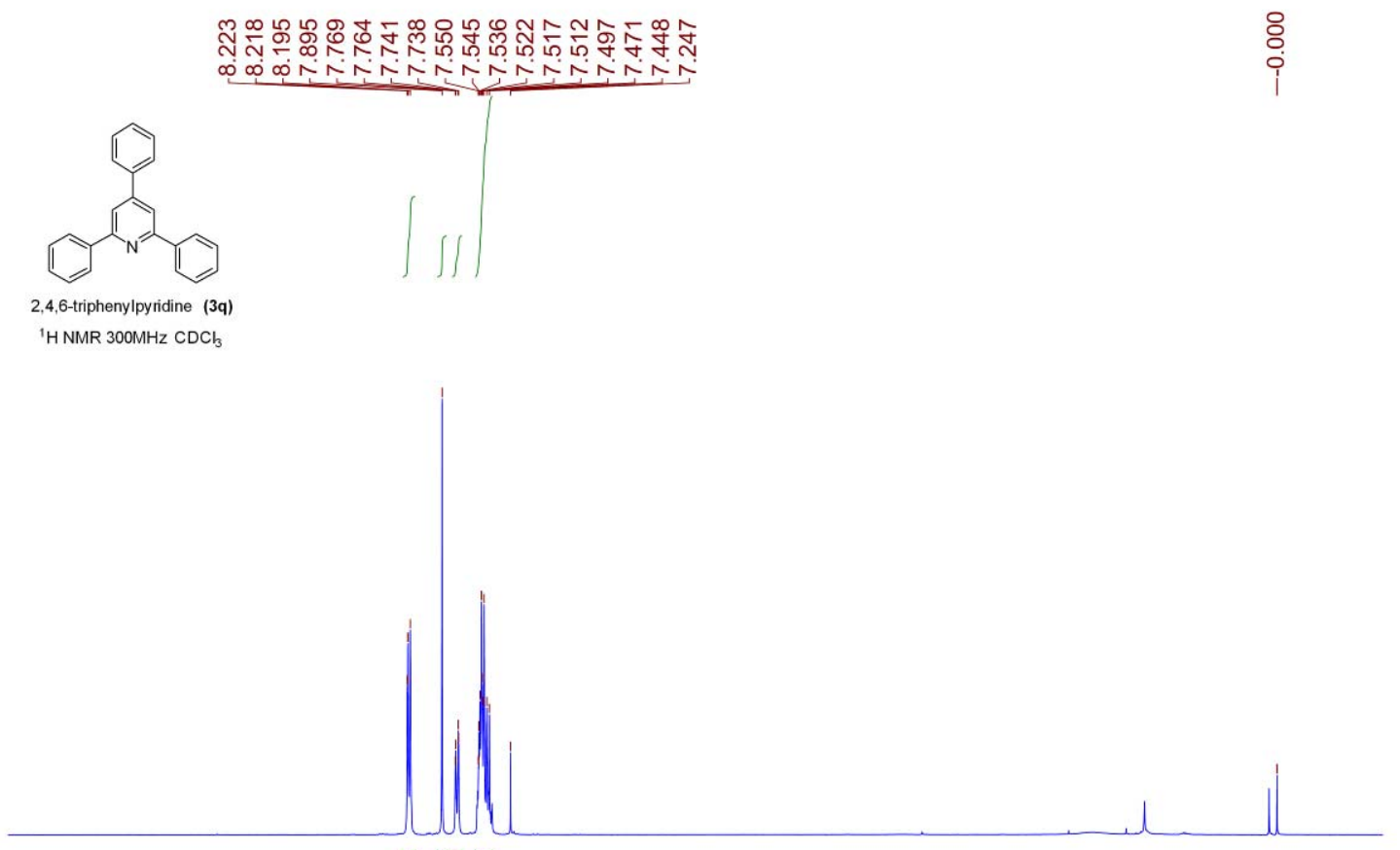

8.8

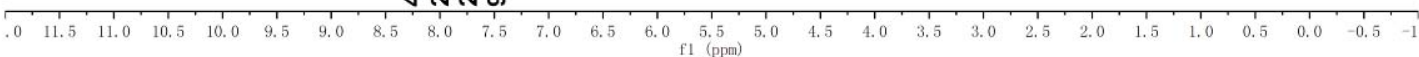

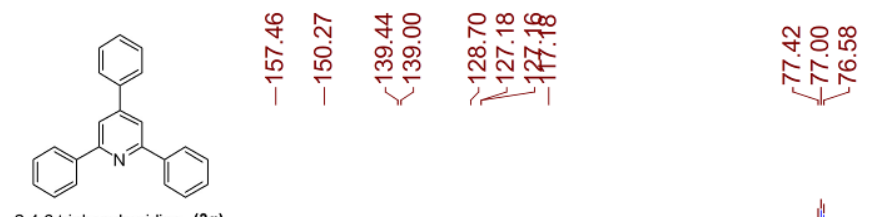

2,4,6-triphenylpyridine (3q)

${ }^{13} \mathrm{C}$ NMR $75 \mathrm{MHz}$ CDCb

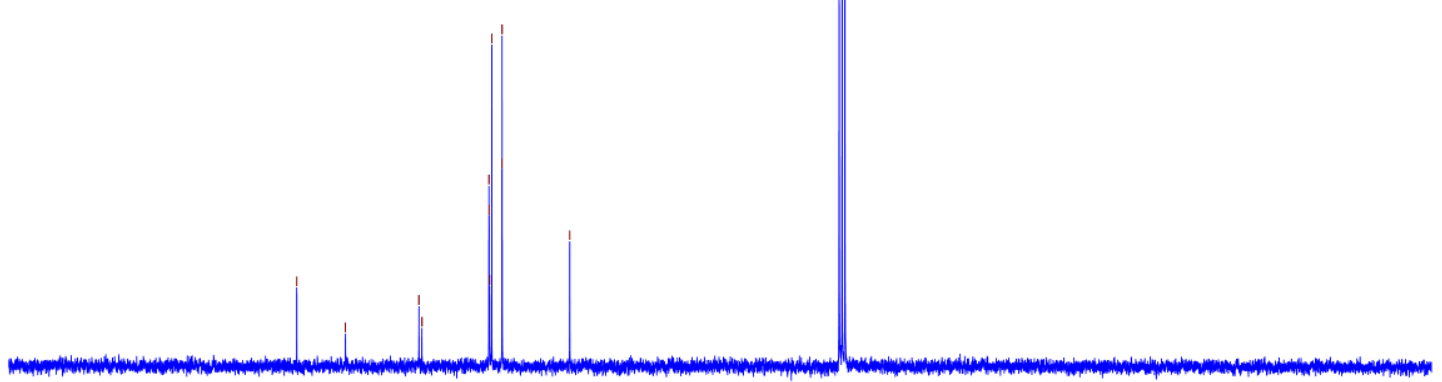

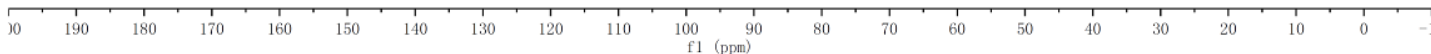




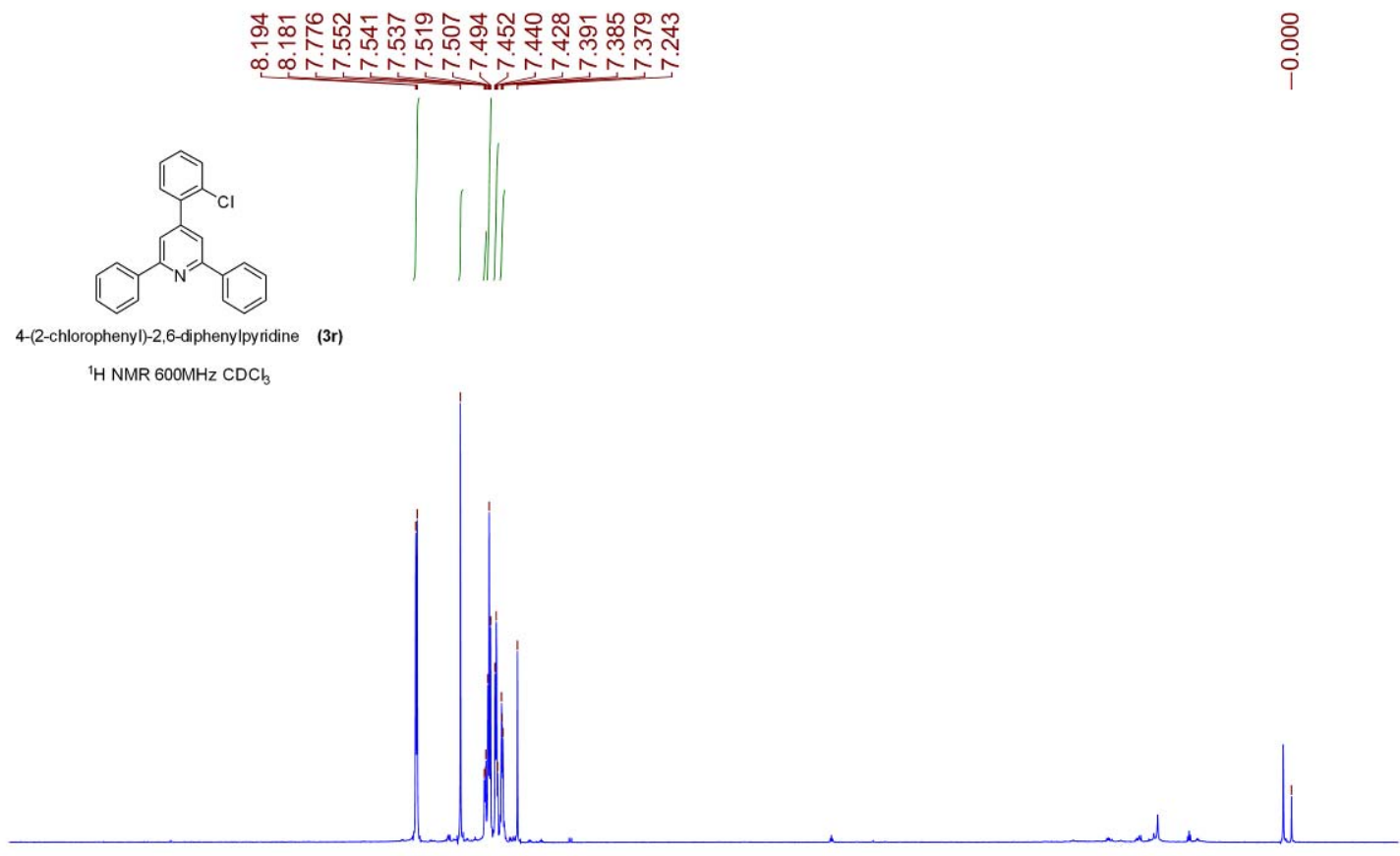

$80080 \%$

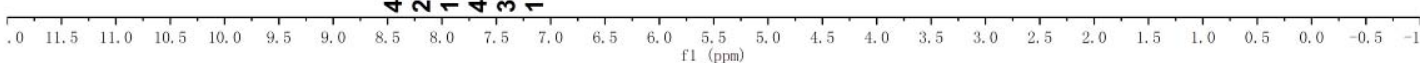

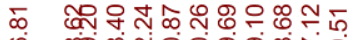

㲅 商

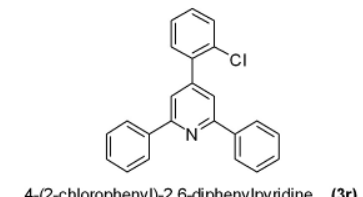

4-(2-chlorophenyl)-2,6-diphenylpyridine (3r)

${ }^{13} \mathrm{C} \mathrm{NMR} 150 \mathrm{MHz}$ CDCl
다요

奀采

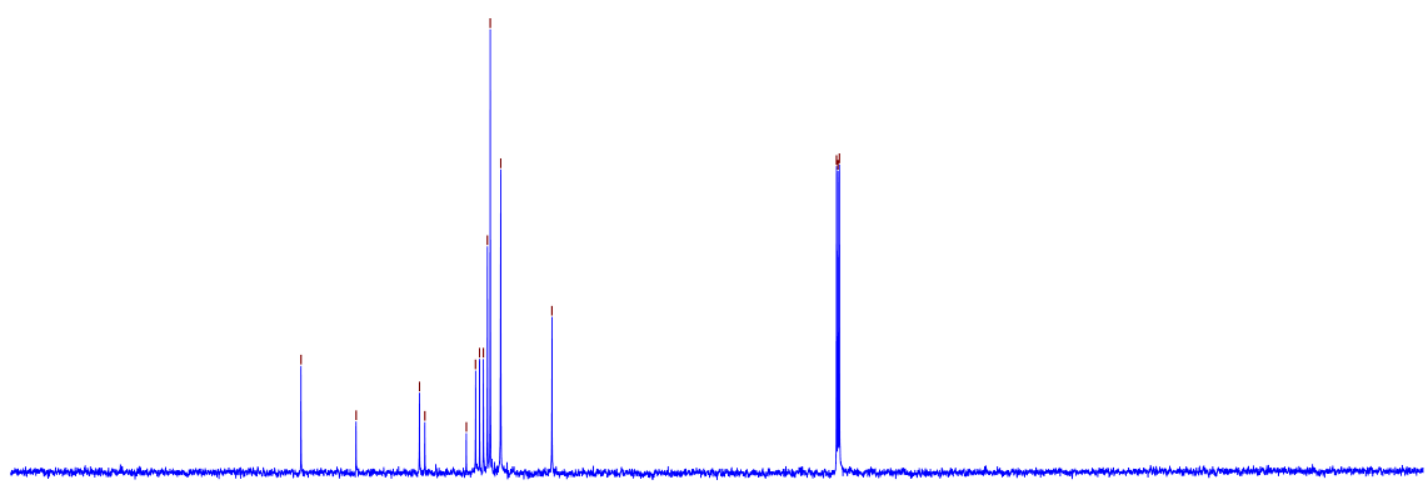

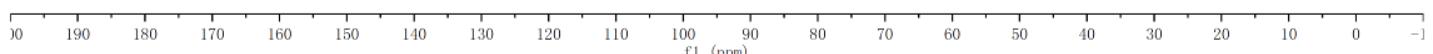




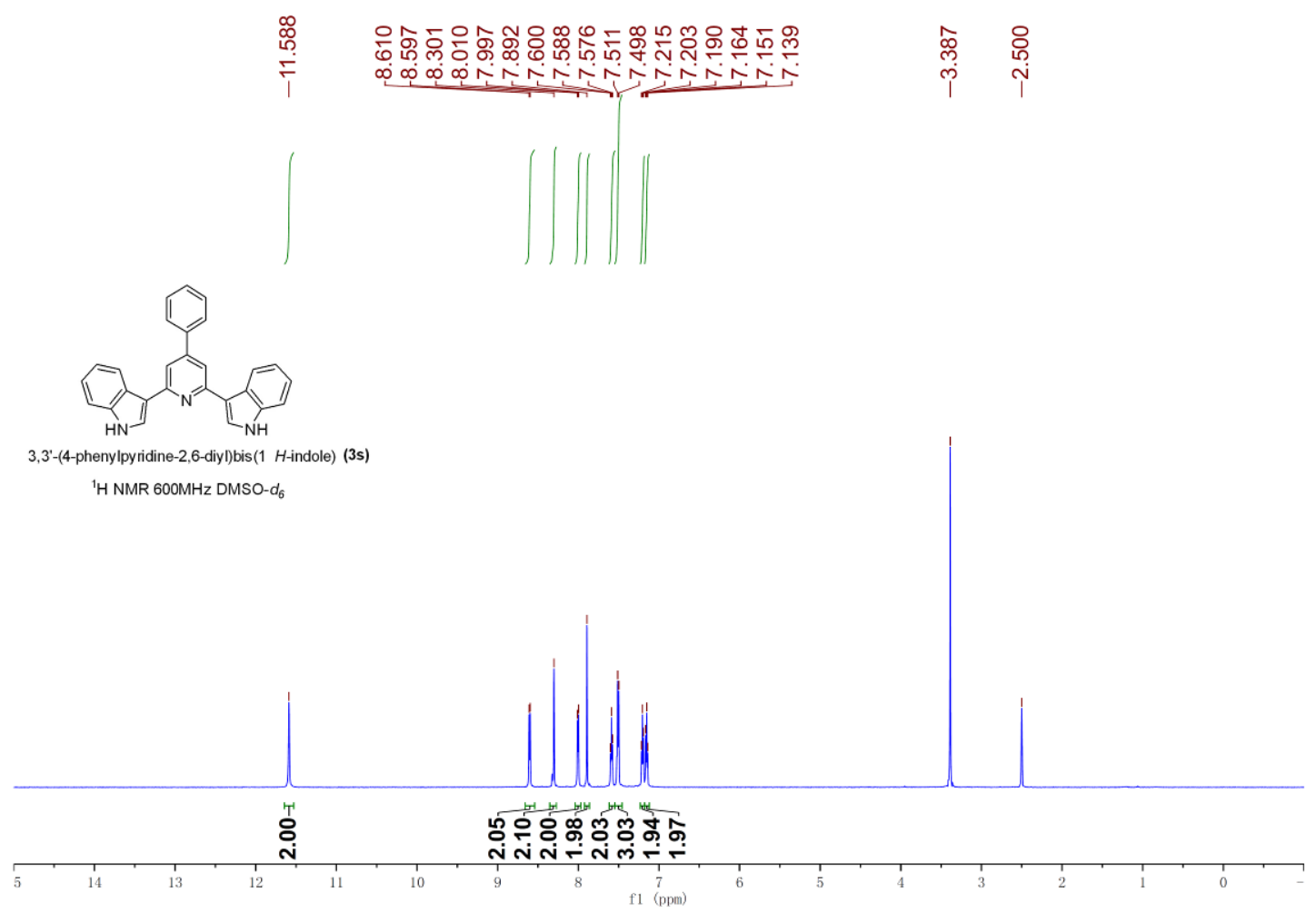

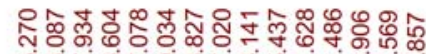

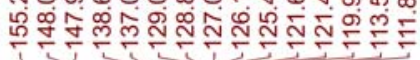

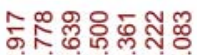

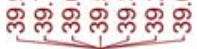

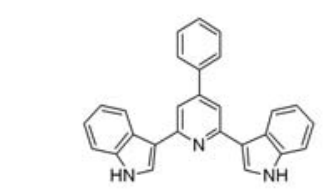

3,3-(4-phenylpyridine-2,6-diy) bis (1H-indole) (3s)

${ }^{13} \mathrm{C}$ NMR $150 \mathrm{MHz}$ DMSO-d

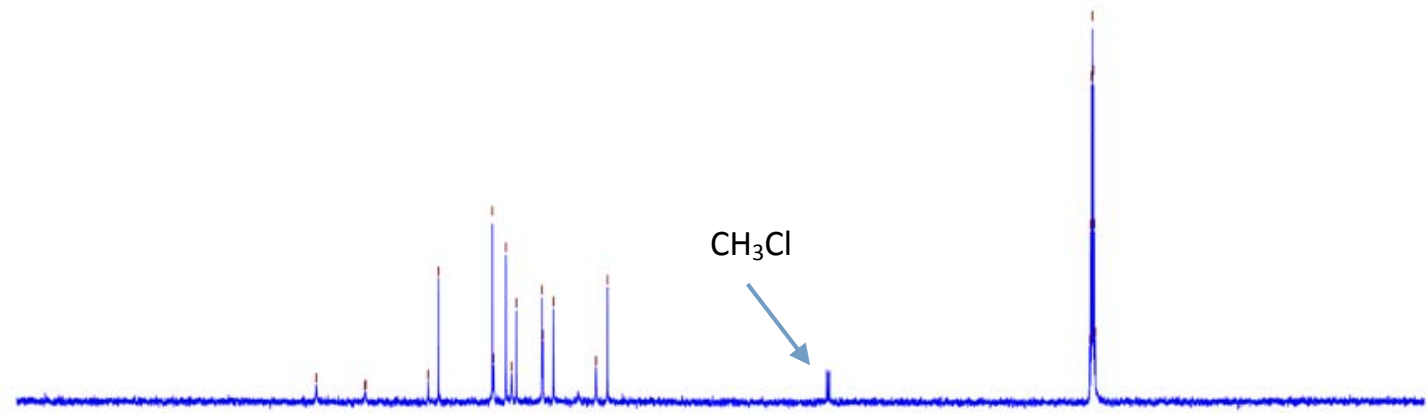

\begin{tabular}{llllllllllllllllllllllllll}
\hline 1 & 190 & 180 & 170 & 160 & 150 & 140 & 130 & 120 & 110 & 100 & 90 & 80 & 70 & 60 & 50 & 40 & 30 & 20 & 10 & 0 & -1
\end{tabular} 


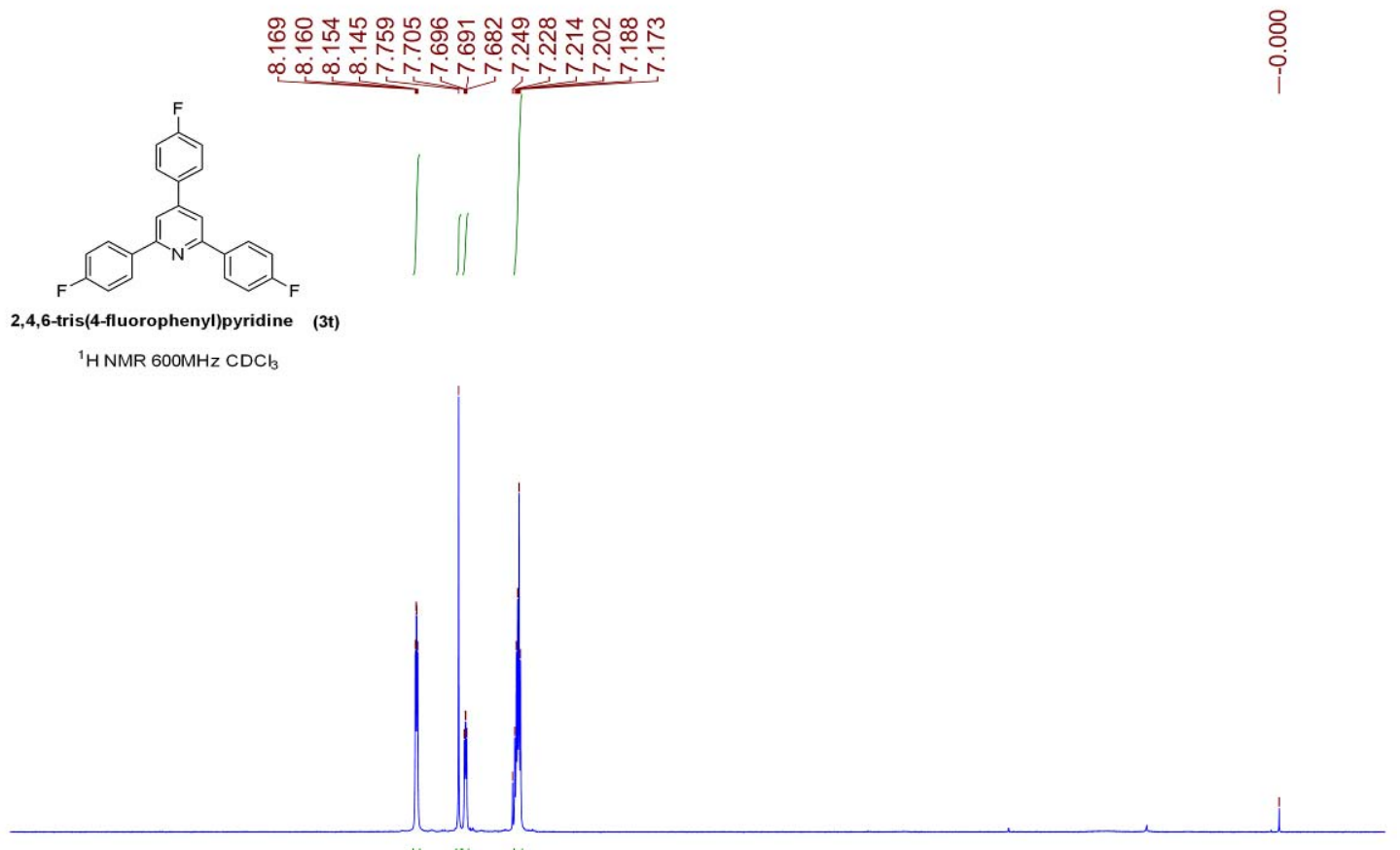
s

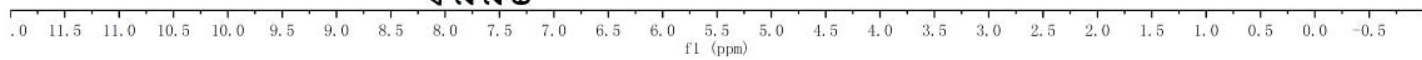

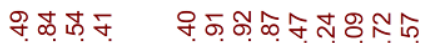

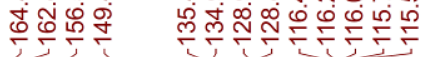

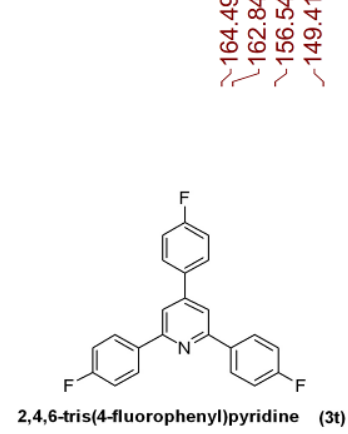

${ }^{13} \mathrm{C} \mathrm{NMR} 150 \mathrm{MHz} \mathrm{CDCl}_{3}$

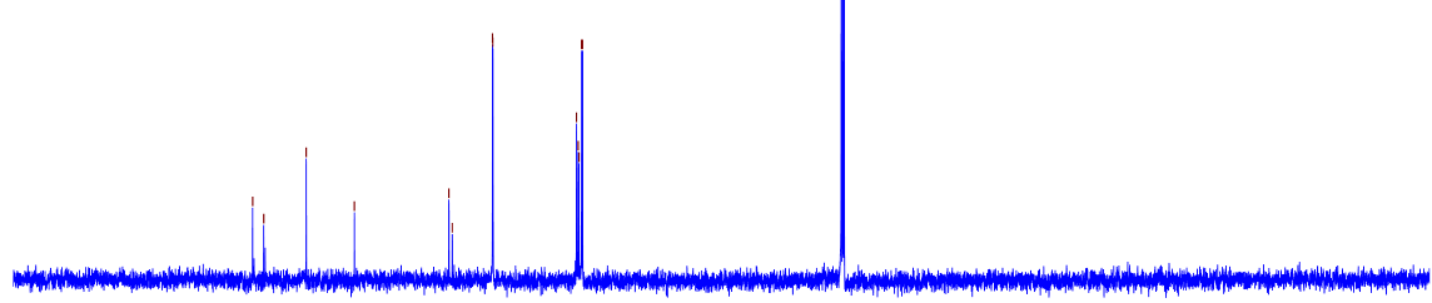

\begin{tabular}{llllllllllll}
\hline 10 & 190 & 180 & 170 & 160 & 150 & 140 & 130 & 120 & 110 & 100 & 90
\end{tabular} 


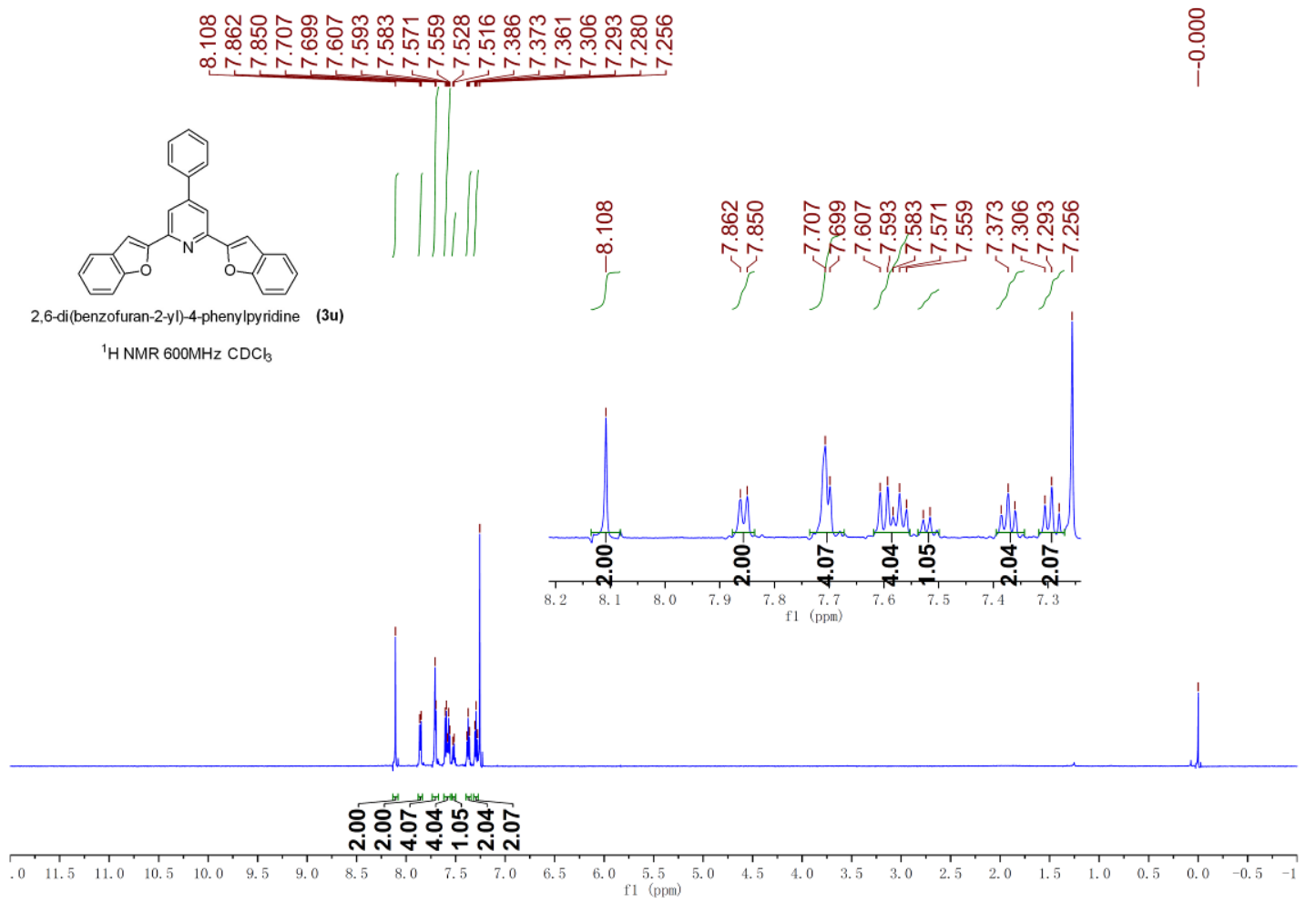

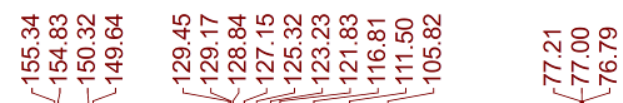

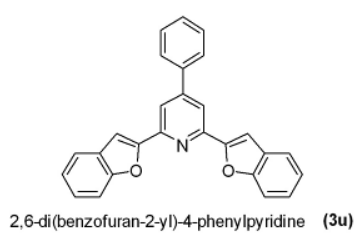

${ }^{13} \mathrm{C} \mathrm{NMR} 150 \mathrm{MHz} \mathrm{CDCl}_{3}$ 

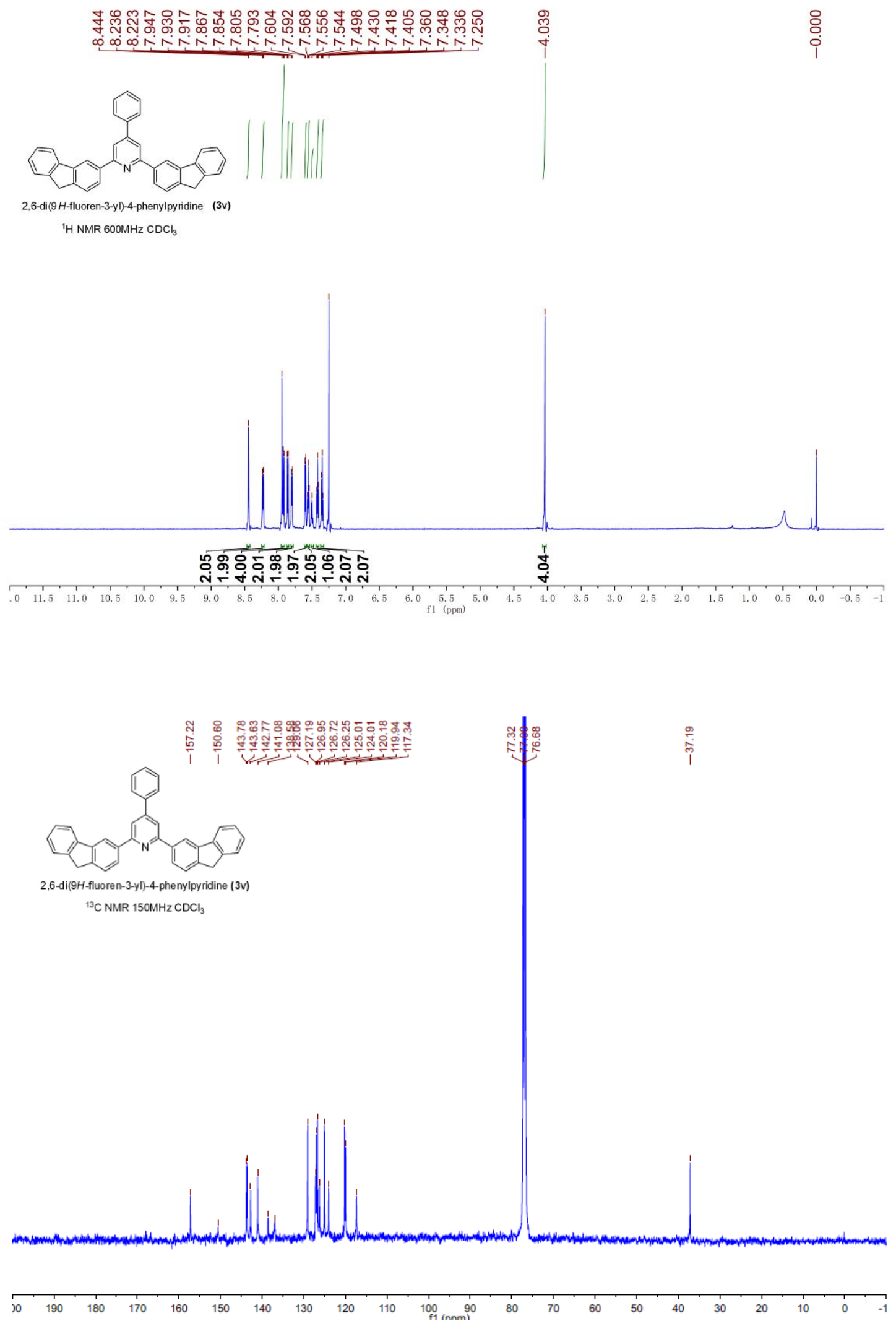


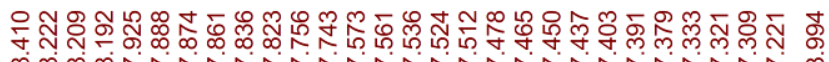

o

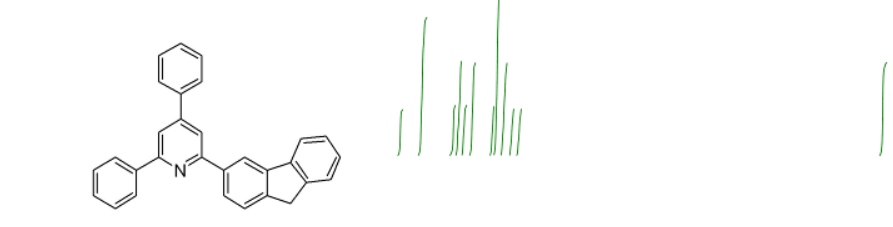

2-(9H-fluoren-3-yl)-4,6-diphenylpyridine (3qv)

${ }^{1} \mathrm{H} \mathrm{NMR} 600 \mathrm{MHz} \mathrm{CDCl}_{3}$

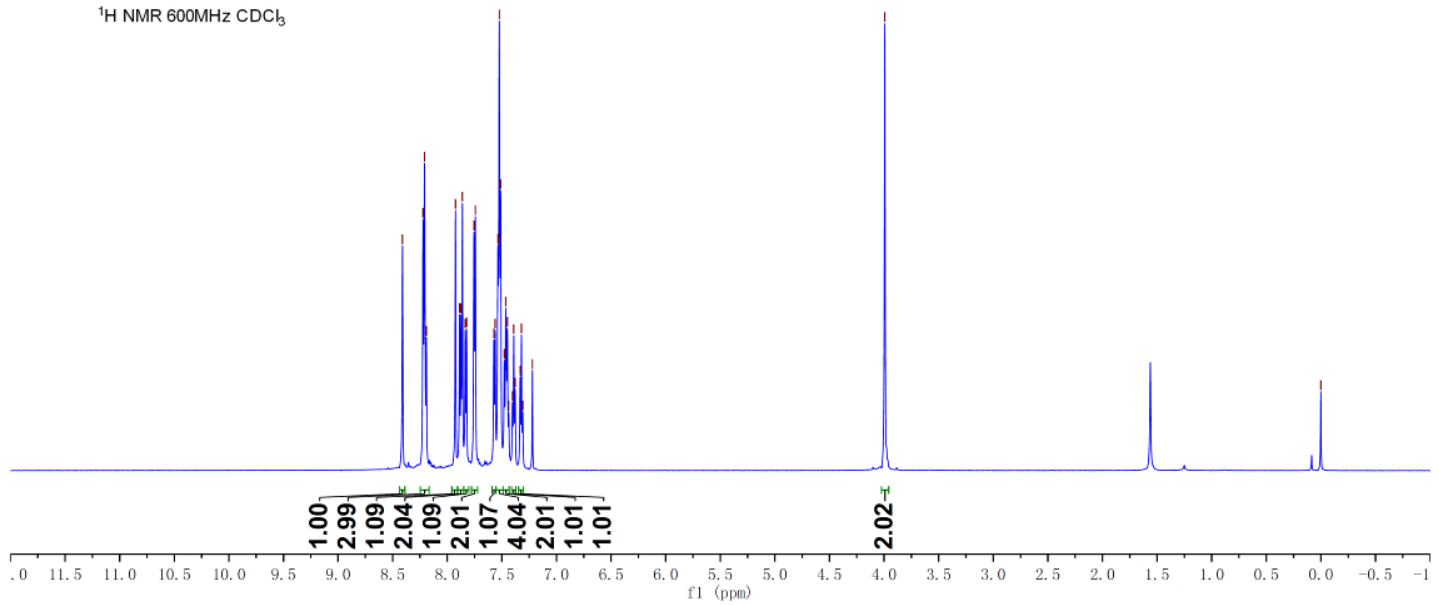

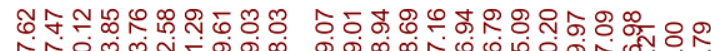

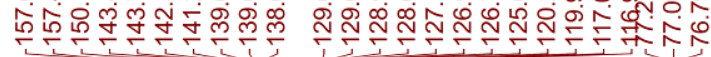

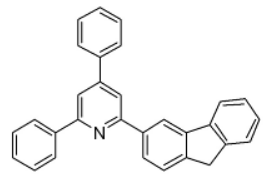

2-(9H-fluoren-3-yl)-4,6-diphenylpyridine (3qv)

${ }^{13} \mathrm{C}$ NMR $150 \mathrm{MHz} \mathrm{CDCl}$

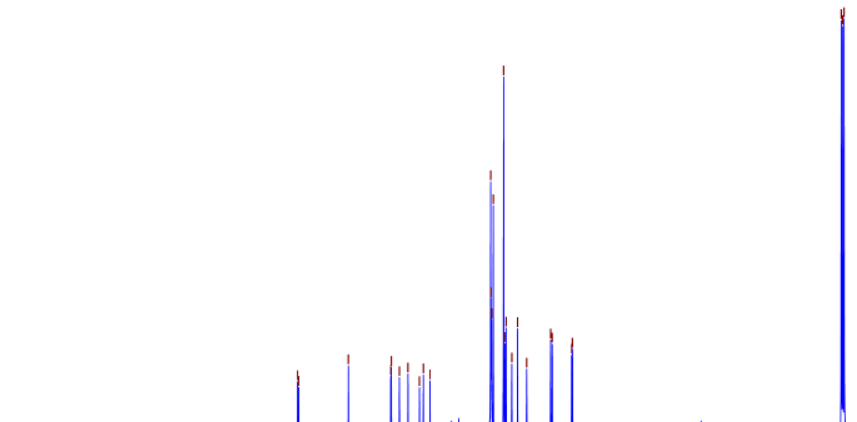

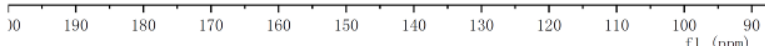




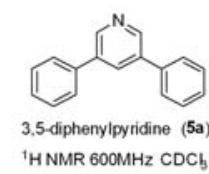

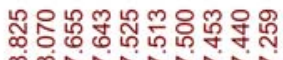

ylpyridine (5a)

innNNNNN
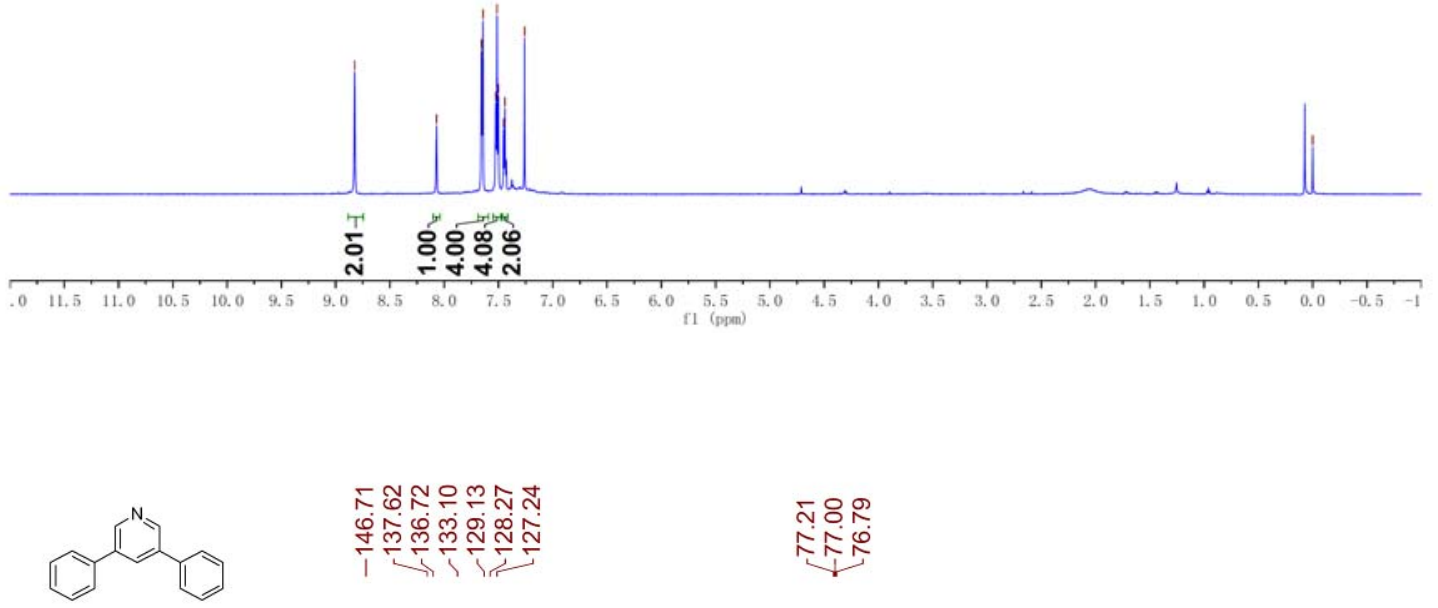

주소음꺾

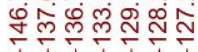

ำำ

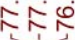

3,5-diphenylpyridine $(5 \mathrm{a})$

${ }^{13} \mathrm{C}$ NMR $150 \mathrm{MHz} \mathrm{CDC}$

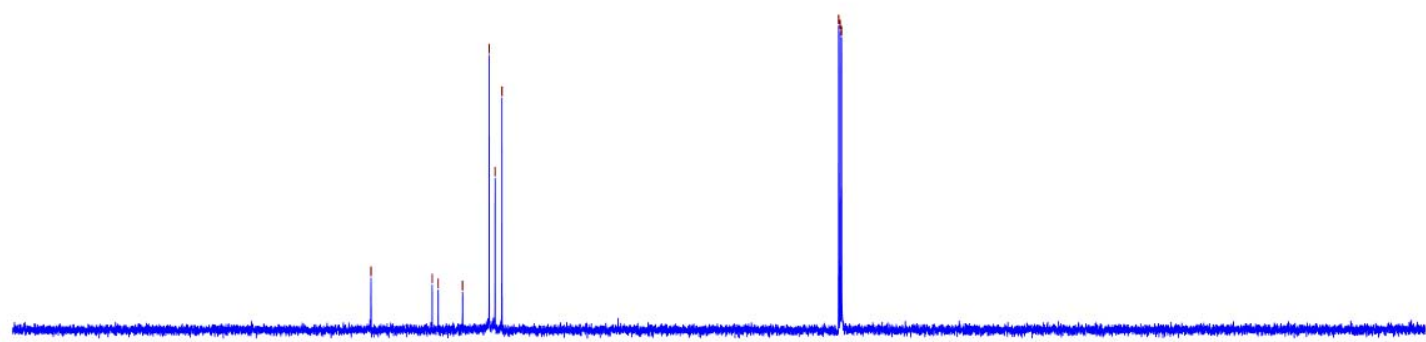

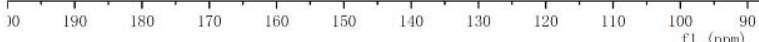



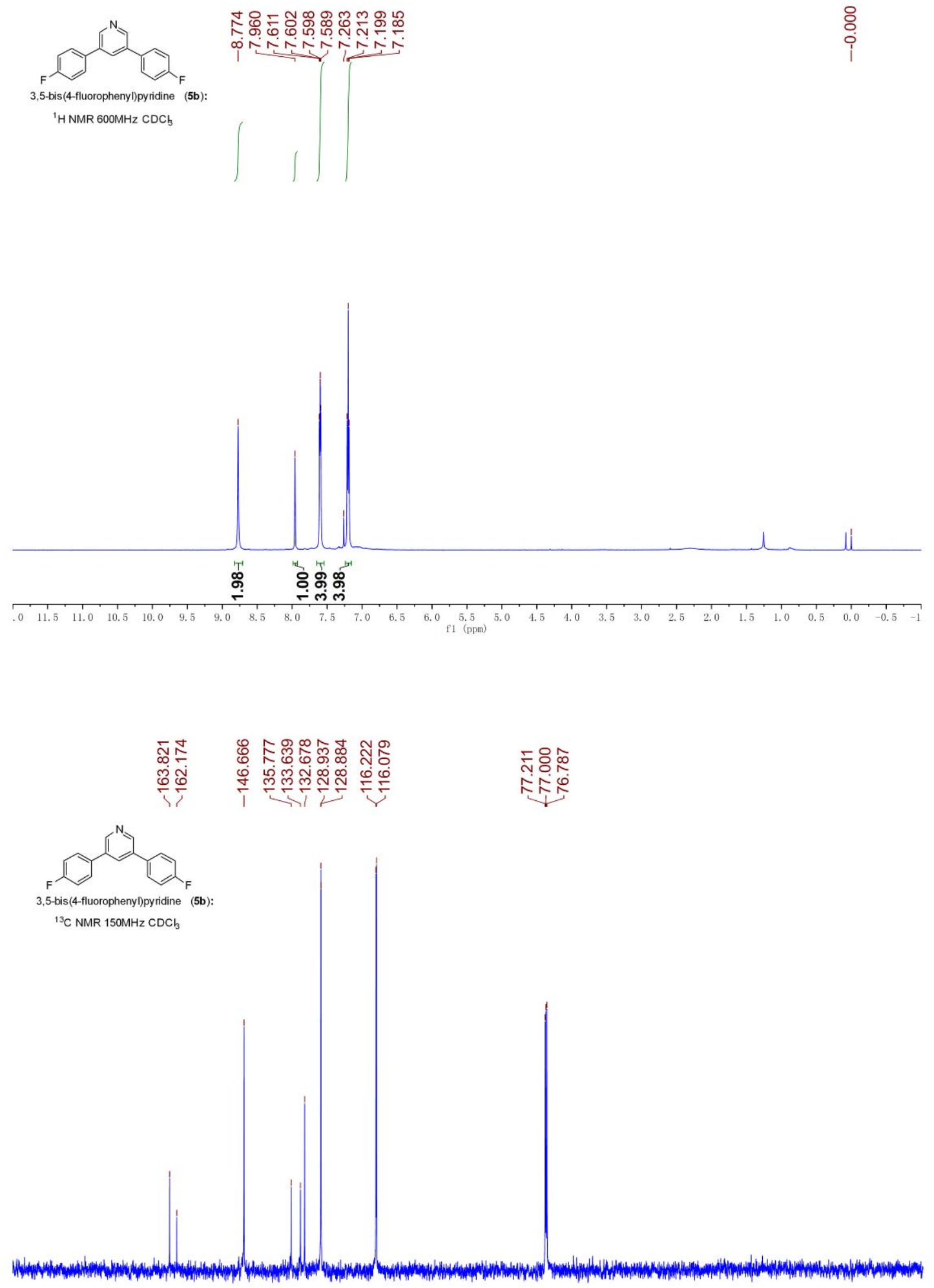

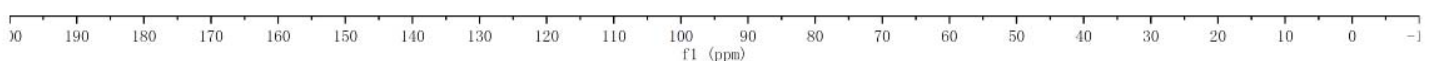



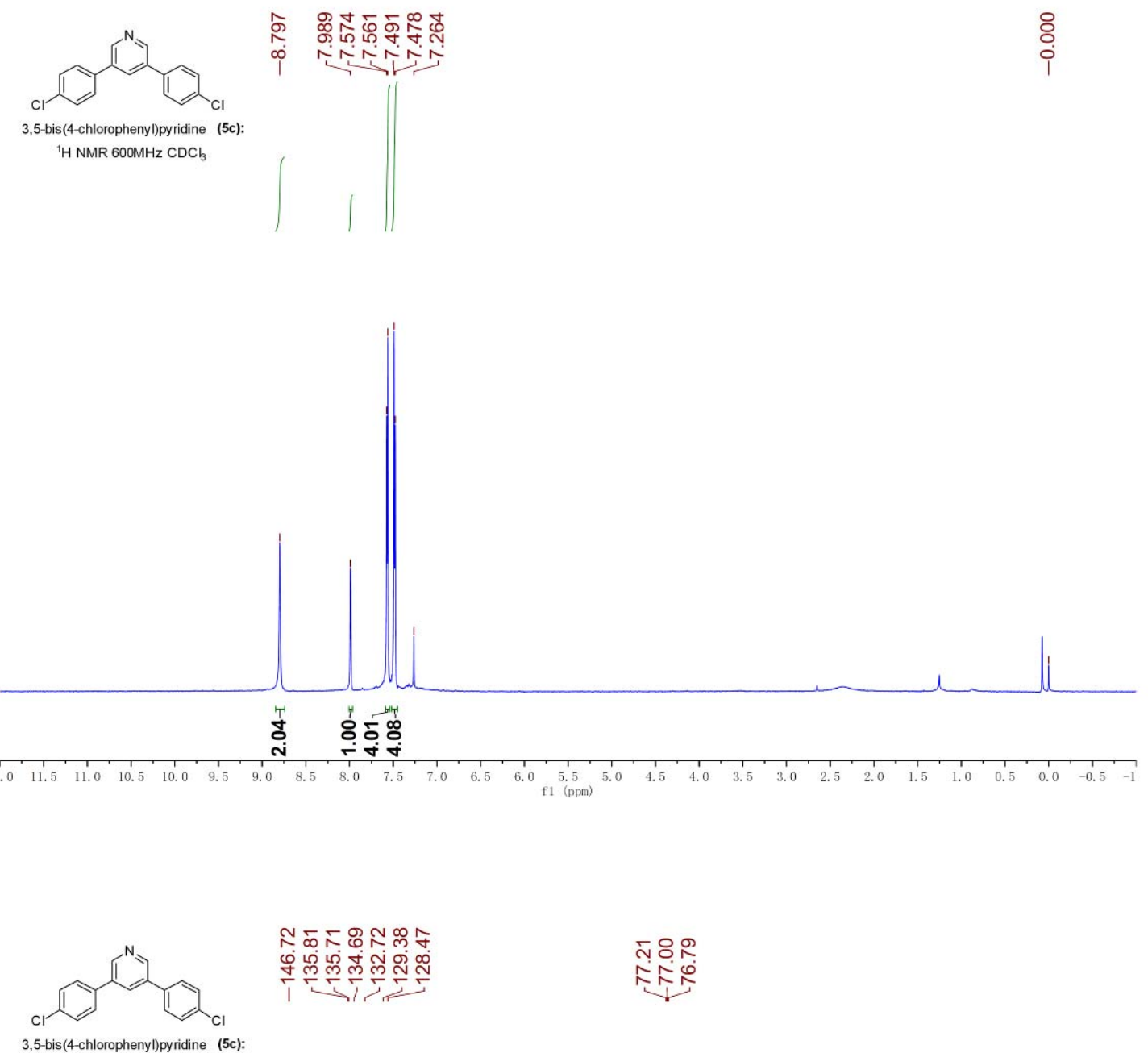

ำธำำ

它

ำ

।

손

3.5-bis(4-chlorophenylpyridine (5c):

${ }^{13} \mathrm{C} \mathrm{NMR} 150 \mathrm{MHz} \mathrm{CDCl}$

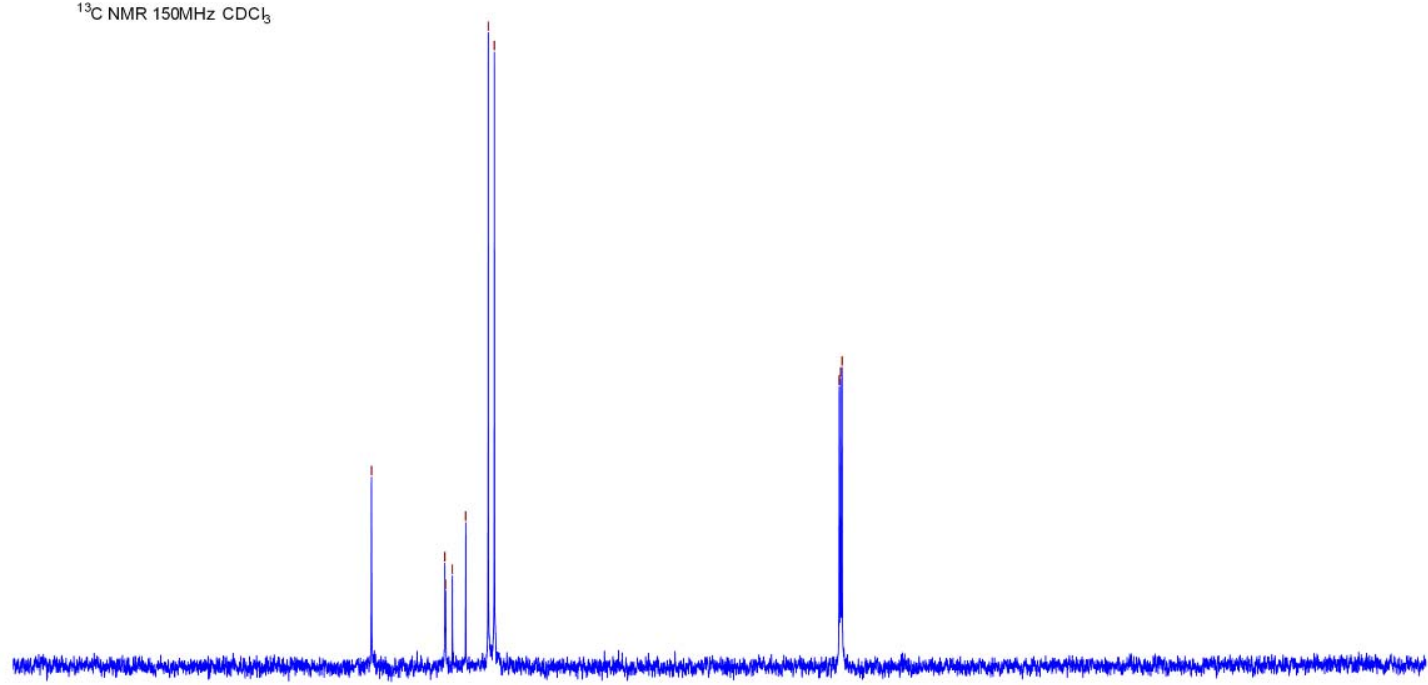

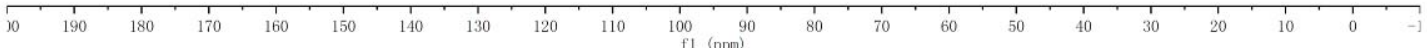



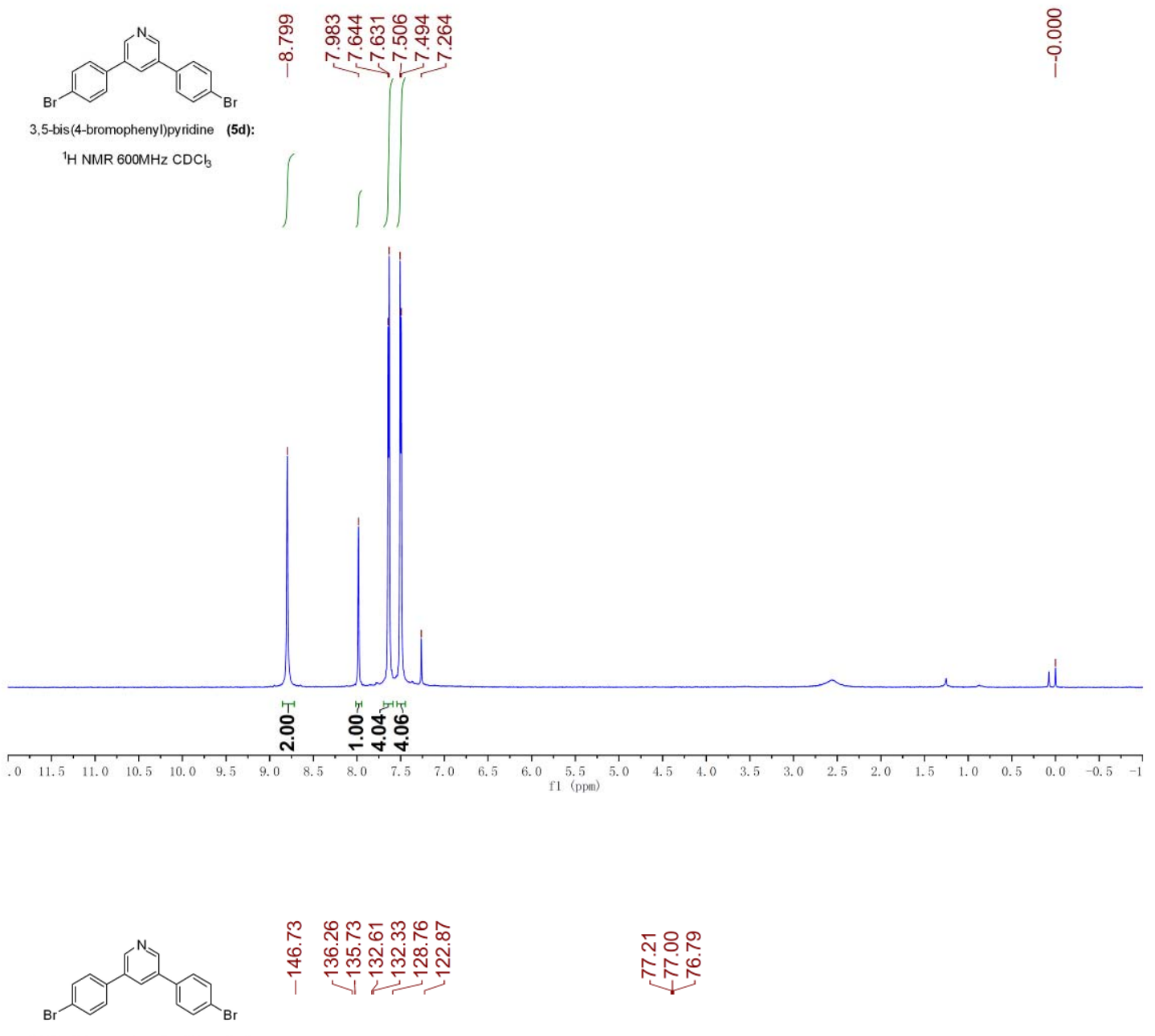

두요

사에

3,5-bis(4-bromophenyl)pyridine (5d):

${ }^{13} \mathrm{C} \mathrm{NMR} 150 \mathrm{MHz} \mathrm{CDCl} 3$

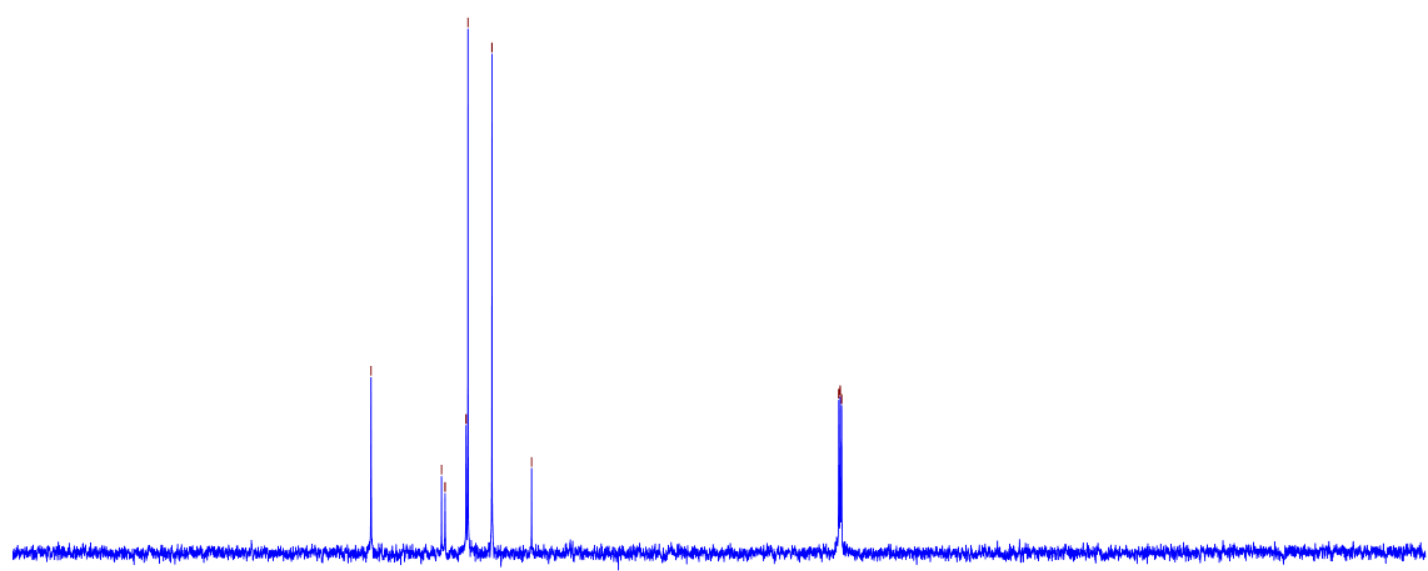

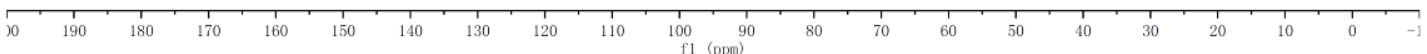




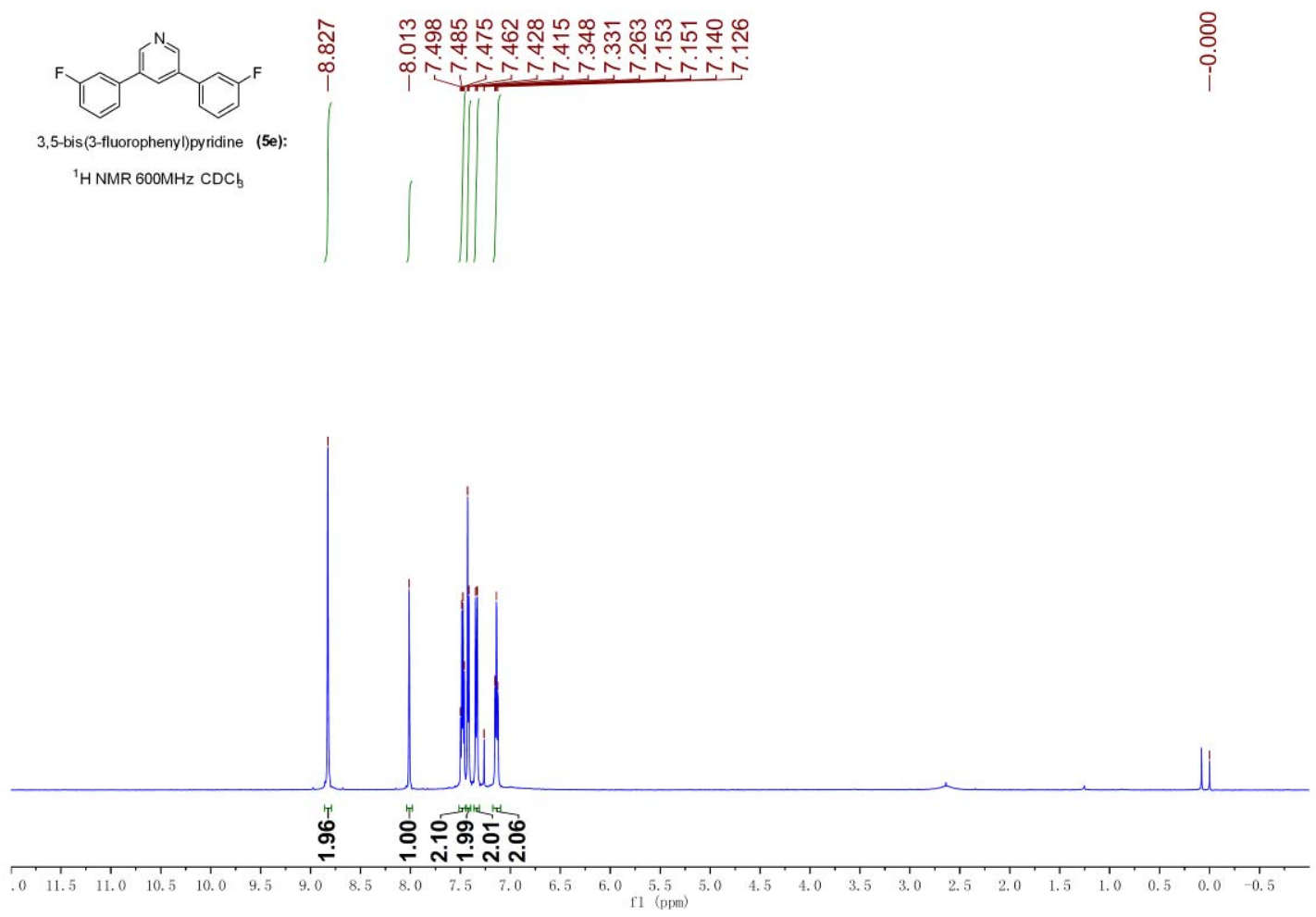

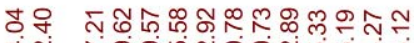

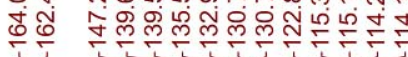

당ำ

kर्ज

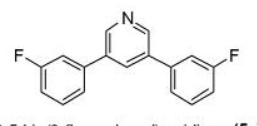

3,5-bis(3-fluorophenyl)pyridine (5e):

${ }^{13} \mathrm{C} \mathrm{NMR} 150 \mathrm{MHz} \mathrm{CDCl}$

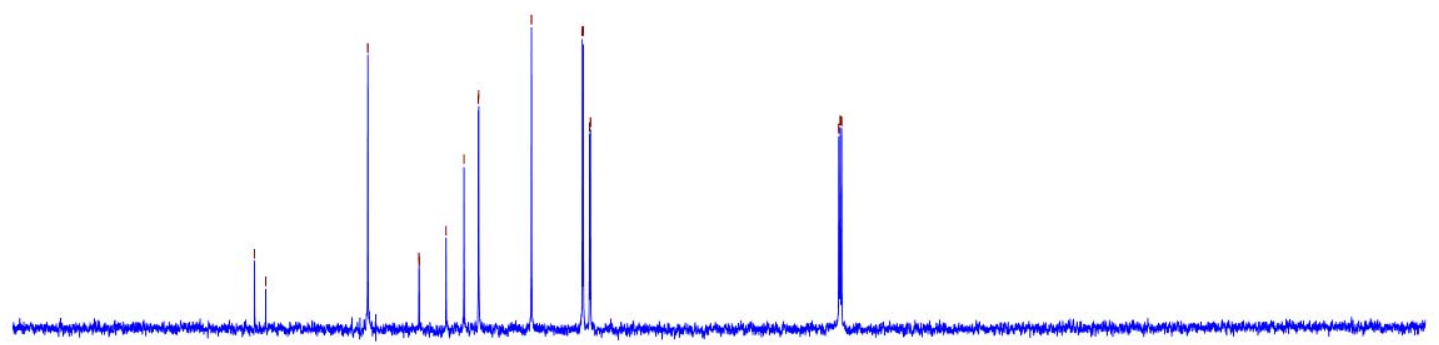

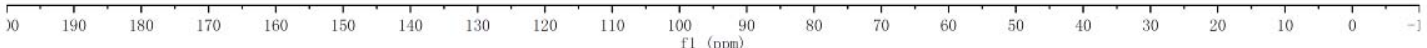



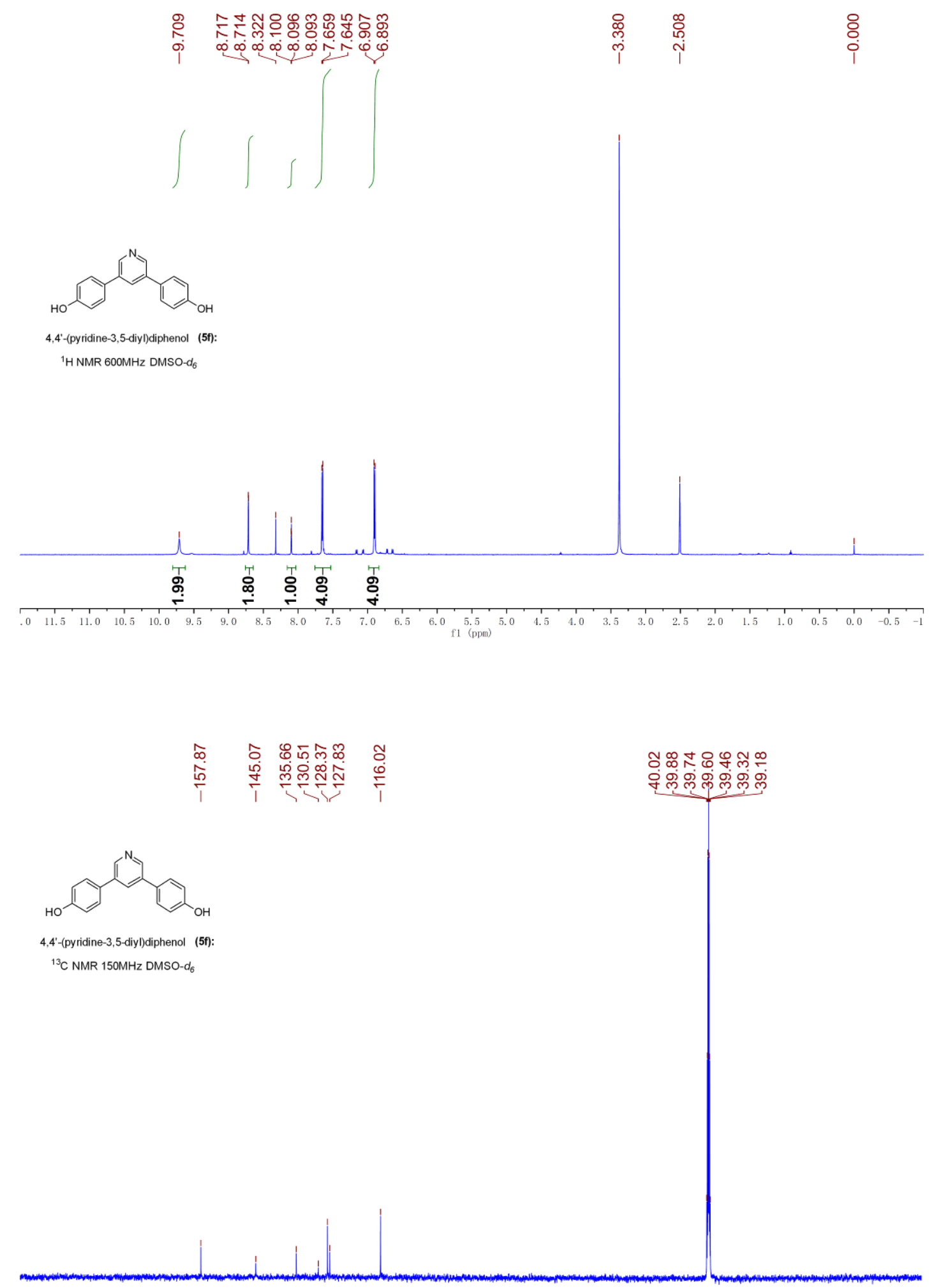

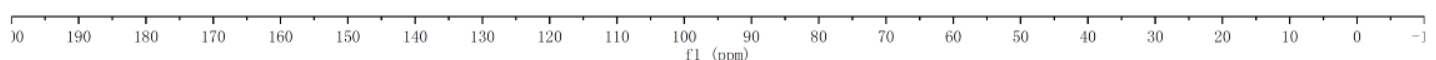



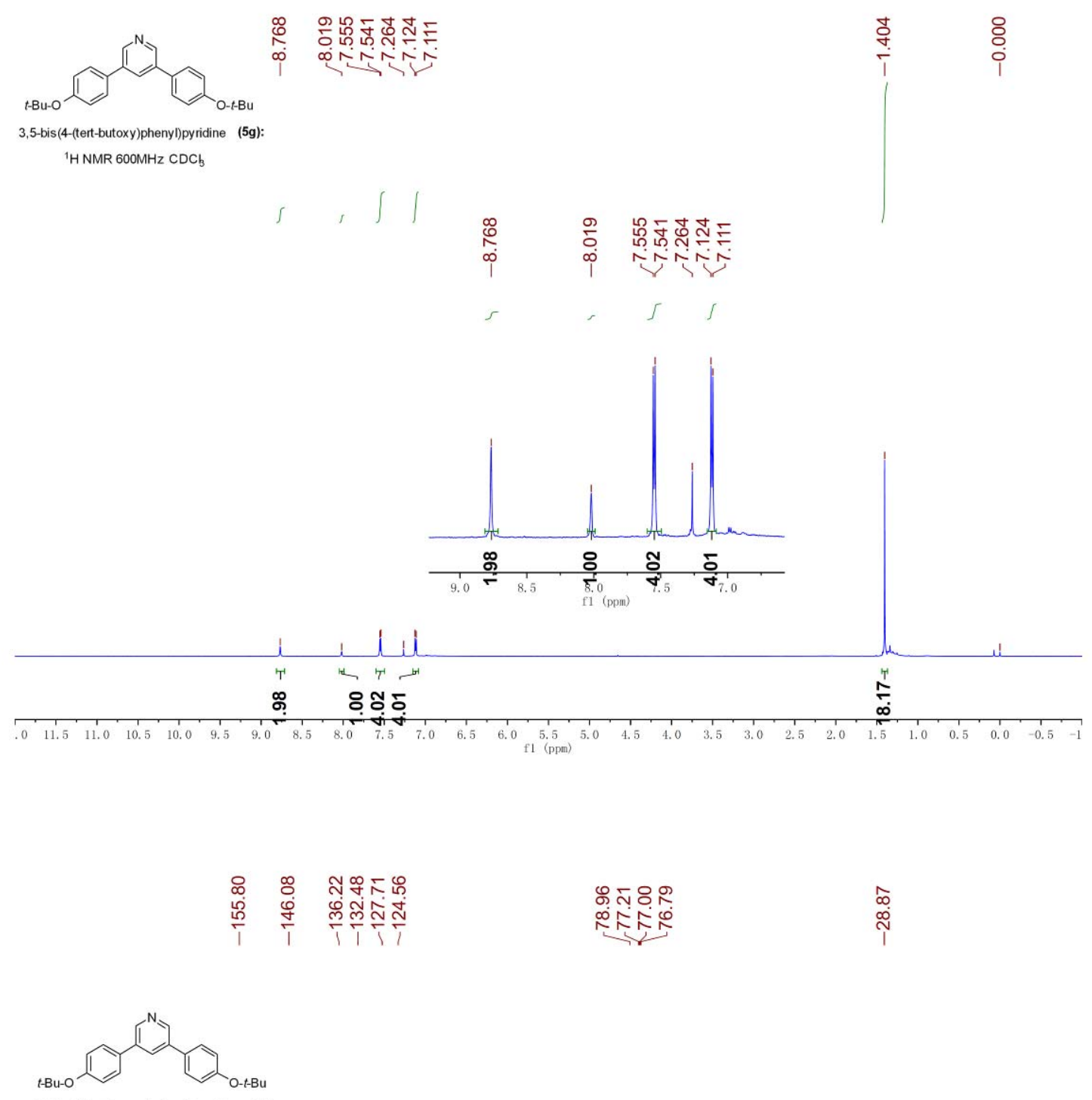

3,5-bis(4-(tert-butoxy)phenyl)pyridine $(\mathbf{5 g})$ : ${ }^{13} \mathrm{C}$ NMR $150 \mathrm{MHz} \mathrm{CDCl}$

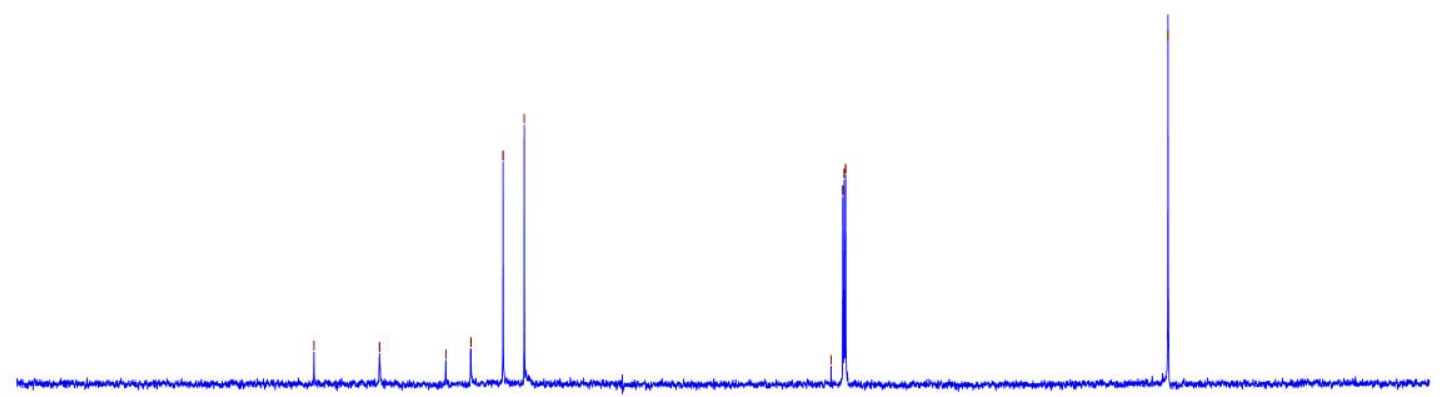

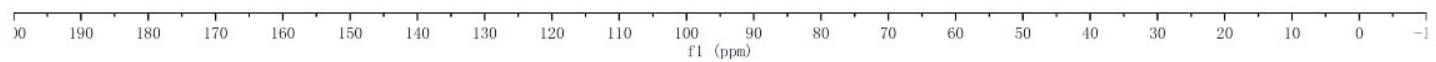

